\title{
CONVERGENCE OF FULLY DISCRETE SCHEMES FOR DIFFUSIVE DISPERSIVE CONSERVATION LAWS WITH DISCONTINUOUS COEFFICIENT
}

\author{
Rajib Dutta ${ }^{1}$, UjJwal Koley ${ }^{2}$ and Deep RaY ${ }^{2}$
}

\begin{abstract}
We are concerned with fully-discrete schemes for the numerical approximation of diffusivedispersive hyperbolic conservation laws with a discontinuous flux function in one-space dimension. More precisely, we show the convergence of approximate solutions, generated by the scheme corresponding to vanishing diffusive-dispersive scalar conservation laws with a discontinuous coefficient, to the corresponding scalar conservation law with discontinuous coefficient. Finally, the convergence is illustrated by several examples. In particular, it is delineated that the limiting solutions generated by the scheme need not coincide, depending on the relation between diffusion and the dispersion coefficients, with the classical Kružkov-Oleinik entropy solutions, but contain nonclassical undercompressive shock waves.
\end{abstract}

Mathematics Subject Classification. 35L65, 35L67, 35L25, 65M12, 65M06, 65M08.

Received June 6, 2015. Revised September 5, 2015. Accepted October 20, 2015.

\section{INTRODUCTION}

In this paper, we consider a finite difference method for the vanishing diffusive-dispersive approximations of scalar conservation laws with a discontinuous flux

$$
\begin{cases}u_{t}^{\varepsilon}+f\left(k(x), u^{\varepsilon}\right)_{x}=\mathcal{R}\left[\varepsilon, \mu(\varepsilon) ; u^{\varepsilon}\right], & x \in \mathbb{R} \times(0, T), \\ u^{\varepsilon}(x, 0)=u_{0}(x), & x \in \mathbb{R},\end{cases}
$$

when $\varepsilon>0$ tends to zero with $0<\mu(\varepsilon) \mapsto 0$ as $\varepsilon \mapsto 0$. Here $\mathcal{R}\left[\varepsilon, \mu(\varepsilon) ; u^{\varepsilon}\right]$ is a regularization term, depends upon two parameters $\varepsilon$ and $\mu(\varepsilon)$ referred to as the diffusion and the dispersion coefficients, motivated by the equations of two-phase flow in porous media, $T>0$ is fixed, $u^{\varepsilon}: \mathbb{R} \times[0, T) \mapsto \mathbb{R}$ is the unknown scalar map, $u_{0}$ the initial data, $k: \mathbb{R} \mapsto \mathbb{R}$ is a spatially varying (discontinuous) coefficient, and the flux function $f: \mathbb{R}^{2} \mapsto \mathbb{R}$ is a sufficiently smooth scalar function (see Sect. 2 for the complete list of assumptions).

Motivated by the dynamic capillary pressure [7], we consider in this paper the simplified model

$$
\mathcal{R}\left[\varepsilon, \mu(\varepsilon) ; u^{\varepsilon}\right]=\varepsilon \beta u_{x x}^{\varepsilon}+\mu(\varepsilon) \gamma u_{x x t}^{\varepsilon}
$$

\footnotetext{
Keywords and phrases. Conservation laws, discontinuous flux, diffusive-dispersive approximation, finite difference scheme, convergence, entropy condition, nonclassical shock.

1 Institut für Mathematik, Julius-Maximilians-Universität Würzburg, Campus Hubland Nord, Emil-Fischer-Strasse 30, 97074, Würzburg, Germany. rajib.ami@gmail.com

2 Centre For Applicable Mathematics (CAM), Tata Institute of Fundamental Research, P.O. Box 6503, GKVK post office, 560065 Bangalore, India. ujjwal@math.tifrbng.res.in; deep@math.tifrbng.res.in
} 
with a third-order mixed derivatives term including one time derivative. Here $\beta, \gamma>0$ are fixed parameters. The equation (1.1) along with (1.2) serves as a concrete model of two phase flows in a heterogeneous porous medium.

Moreover, drawing preliminary motivation from phase transition dynamics, we also consider the following specific form of the regularization term

$$
\mathcal{R}\left[\varepsilon, \mu(\varepsilon) ; u^{\varepsilon}\right]=\varepsilon \beta u_{x x}^{\varepsilon}+\mu(\varepsilon) \gamma u_{x x x}^{\varepsilon},
$$

with $\beta, \gamma>0$ fixed.

Furthermore, for the simplicity in the exposition, we assume that the flux function has the following particular form

$$
f(k(x), u)=k(x) f(u) .
$$

Note that the flux $k(x) f(u)$ has a possibly discontinuous spatial dependence through the coefficient $k$, which is allowed to have jump discontinuities.

The scalar conservation laws with a discontinuous flux function

$$
u_{t}+f(k(x), u)_{x}=0
$$

is a special example of this type of problems, corresponds to the case $\beta=\gamma=0$. A simple physical model corresponding to (1.4) is the Witham model of car traffic flow on a highway (consult the monograph by Leveque [22]), where the spatially varying coefficient $k$ corresponds to changing road conditions. Several other models such as two phase flow in a heterogeneous porous medium that arise in petroleum industry, the modeling of the clarifier thickener unit used in waste water treatment plants are also corresponding to (1.4).

Independently of the smoothness of the initial data $u_{0}$ and $k$, solutions to (1.4) are not necessarily smooth due to the presence of nonlinear flux term in the equation (1.4). Thus, weak solutions must be sought.

Definition 1.1 (Weak solution). A weak solution of the initial value problem (1.4) is a bounded measurable function $u: \mathbb{R} \times[0, T) \rightarrow \mathbb{R}$ satisfying

$$
\int_{\mathbb{R}} \int_{0}^{T}\left(\varphi_{t} u+\varphi_{x} k(x) f(u)\right) \mathrm{d} x \mathrm{~d} t+\int_{\mathbb{R}} \varphi(x, 0) u_{0}(x) \mathrm{d} x=0,
$$

for all $\varphi \in C_{0}^{\infty}(\mathbb{R} \times[0, T))$.

It is well-known that (weak) solutions may be discontinuous and they are not uniquely determined by their initial data. Consequently, an entropy condition must be imposed to single out the physically correct solution. If $k(x)$ is "smooth", a weak solution $u$ satisfies the entropy condition if for all convex $C^{2}$ functions $\eta: \mathbb{R} \rightarrow \mathbb{R}$

$$
\eta(u)_{t}+(k(x) Q(u))_{x}+k^{\prime}(x)\left(\eta^{\prime}(u) f(u)-Q(u)\right) \leq 0, \text { in } \mathcal{D}(\mathbb{R} \times[0, T]),
$$

where $Q: \mathbb{R} \rightarrow \mathbb{R}$ is defined by $Q^{\prime}(u)=\eta^{\prime}(u) f^{\prime}(u)$.

By standard limiting argument, this implies the Kružkov-type entropy condition

$$
|u-c|_{t}+\operatorname{sign}(u-c)(k(x)(f(u)-f(c)))_{x}+\operatorname{sign}(u-c) f(c) k^{\prime}(x) \leq 0, \text { in } \mathcal{D}(\mathbb{R} \times[0, T]),
$$

holds for all $c \in \mathbb{R}$.

However, the notion of entropy solution described above breaks down when $k(x)$ is discontinuous. In view of [14], we use the following notion of entropy solution for (one-dimensional) conservation laws with discontinuous flux equations with coefficients that are only spatially dependent. We assume that the spatially varying coefficient $k(x)$ is piecewise $C^{1}$ with finitely many jumps (in $k$ and $k^{\prime}$ ), located at $\xi_{1}, \xi_{2}, \ldots, \xi_{M}$. 
Definition 1.2 (Entropy solution). A weak solution $u$ of the initial value problem (1.4) is called an entropy solution, if the following Kružkov-type entropy inequality holds for all $c \in \mathbb{R}$ and all test functions $0 \leq \psi \in$ $\mathcal{D}(\mathbb{R} \times[0, T])$.

$$
\begin{aligned}
& \int_{\mathbb{R}} \int_{0}^{T}\left(|u-c| \psi_{t}+\operatorname{sign}(u-c) k(x)(f(u)-f(c)) \psi_{x}\right) \mathrm{d} x \mathrm{~d} t+\int_{\mathbb{R}}\left|u_{0}-c\right| \psi(x, 0) \mathrm{d} x \\
& \quad+\int_{\mathbb{R} \backslash\left\{\xi_{m}\right\}_{m=1}^{M}} \int_{0}^{T} \operatorname{sign}(u-c) k^{\prime}(x) f(c) \psi \mathrm{d} x \mathrm{~d} t+\sum_{m=1}^{M} \int_{0}^{T}\left|f(c)\left(k_{m}^{+}-k_{m}^{-}\right)\right| \psi\left(\xi_{m}, t\right) \mathrm{d} t \geq 0 .
\end{aligned}
$$

The last couple of decades have witnessed remarkable advances in the studies of conservation laws with discontinuous flux function. However, we will not be able to discuss the whole literature here, but only refer to the parts that are pertinent to the current paper. In case of "smooth" $k(x)$, the notion of entropy solution was introduced independently by Kružkov [19] and Vol'pert [32] (the latter author considered the smaller BV class). These authors also proved general existence, uniqueness, and stability results for the entropy solution, see also Olënik [25] for similar results in the convex case $f_{u u} \geq 0$.

\subsection{Diffusive dispersive approximation}

It is well-known that the conservation law (1.4) is derived by neglecting underlying small scale effects such as diffusion, dispersion, capillarity etc., and may admit physically relevant discontinuous solutions containing shock waves (non-classical shock) that may depend on underlying small-scale mechanisms. It has been successively recognized that a standard entropy inequality (due to Kružkov, Olënik, and others) does not suffice to single out such a physically relevant solution, and it is important to incorporate these small-scale effects in the entropy condition. In other words, additional admissibility criteria (a kinetic relation) are required in order to characterize these small-scale dependent non classical shock waves uniquely. In [9], the authors developed a framework for the existence and uniqueness of the non-classical shock waves that arise as limits of diffusivedispersive approximations.

Noting that the solutions to (1.4) can be different due to their explicit dependence on the underlying small scale effects, we focus on a concrete model of two phase flow in porous medium (for a brief derivation of this model consult [4]). The relevant small scale effect is a dynamic capillary pressure term, that was introduced in [7]. Compared to the standard capillary pressure models [1], the addition of the new term resulted in a model that contain higher-order mixed spatio-temporal derivatives ( $c f .(1.2))$.

The diffusive-dispersive model has a long tradition, starting with the analysis of linear diffusion-dispersion model (1.1). A pioneering study of the effect of vanishing diffusion and dispersion terms in scalar conservation laws, with $x$-independent flux function, can be found in Schonbek [27]. The technique of compensated compactness was used to prove convergence toward weak solutions. Kondo and LeFloch [18] studied zero diffusiondispersion limits for $x$-independent fluxes under an optimal balance between the sizes of the diffusion and dispersion parameters. LeFloch and Natalini [20] used the concept of measure-valued solution and established convergence results assuming that the diffusion dominates the dispersion. Subsequently, the approach of kinetic decomposition and velocity averaging [26] was introduced by Hwang and Tzavaras [11] to analyze singular limits including nonclassical shock waves. Moreover, we also mention related works by Wu [33] and Jacobs, McKinney, and Shearer [12] which provides the first existence result of undercompressive shocks for the modified Kortewegde Vries-Burgers equation. Furthermore, conservation laws with spatially discontinuous flux that are perturbed by diffusion and dispersion terms has been studied by Kissling and Karlsen in [17].

It is well-known that the relative scaling between $\varepsilon$ and $\mu(\varepsilon)$ determines the limiting behavior of solutions, and we can distinguish between three cases:

- Diffusion-dominant regime $\mu(\varepsilon) \ll \varepsilon^{2}$ : the qualitative behavior of solution is same as the solution of the conservation laws. 
- Dispersion-dominant regime $\mu(\varepsilon) \gg \varepsilon^{2}$ : In this case, high oscillations develop (as $\varepsilon \downarrow 0$ ) especially in regions of steep gradients of the solutions and only weak convergence is observed.

- Balanced diffusion-dispersion regime: This typically corresponds to the scenario where $\mu(\varepsilon)=\mathcal{O}\left(\varepsilon^{2}\right)$. Only mild oscillations are observed near shocks, and the limit solution is a weak solution to conservation laws. Most importantly, in this case, the solution exhibit non-classical behavior, as they contain undercompressive shocks. However, for the regime $\mu(\varepsilon)=\mathcal{O}\left(\varepsilon^{2}\right)$, the limit solution coincides with the entropy solution determined by Kružkov theory [19].

\subsection{Numerical schemes}

It is well-known that standard finite difference, finite volume and finite element methods have been very successful in computing solutions to hyperbolic conservation laws with discontinuous coefficients, including those containing shock waves. However, we mention that most of these well-established methods are proven to be not good enough to capture nonclassical shock wave solutions numerically. This well-known phenomena has been explained by many authers (Hou and LeFloch [10], Hayes and LeFloch [8], and others) in terms of the equivalent equation associated with discrete schemes through a formal Taylor expansion. The key idea behind capturing nonclassical shocks is to design finite difference schemes whose equivalent equation matches, both, the diffusive and the dispersive terms (cf. (1.3)) in the underlying model. However, these schemes fail to approximate nonclassical solutions with large amplitude, especially strong shocks due to lack of control on higher order error terms present in equivalent equation. A recent work by Ernest et al. [6] has overcome such problems by dominating higher order error terms in amplitude by the leading order terms of the equivalent equation.

In another paper by Chalons and Lefloch [2], the authors introduced a fully -discrete scheme for the numerical approximation of diffusive-dispersive hyperbolic conservation laws ( $c f .(1.1)-(1.3))$ in one-space dimension. An important feature of their scheme is that it satisfies a cell entropy inequality and, as a consequence, the space integral of the entropy is a decreasing function of time. Moreover, they showed that the limiting solutions generated by the scheme contains nonclassical undercompressive shock waves.

On the other hand, there is a sparsity of efficient numerical schemes for (1.1) and (1.2) available in the literature. In fact, to the best of our knowledge, this is the first systematic attempt to construct a provably convergent numerical scheme for (1.1) and (1.2). Having said this, there are some numerical experiments available in the final section of the recent paper by Coclite et al. [4] without rigorous analysis of the scheme.

\subsection{Scope and outline of the paper}

In view of the above discussion, it is fair to claim that there are no robust and provably stable numerical schemes currently available to simulate the vanishing capillarity approximations of scalar conservation laws equation (1.1) and (1.2). In this context, we consider a fully-discrete (in both space and time) finite difference scheme for (1.1) and (1.2) which is provably convergent and able to capture non classical shocks quite well. Since diffusion-dispersion model for the conservation laws with discontinuous flux has not been studied in detail, we analyze a fully-discrete scheme for (1.1) and (1.3) as well. While there are several numerical methods which perform well in practice, perhaps better than the one presented here, (see [21] for a recent comparison of diferent numerical methods) we emphasize that we prove the convergence of the schemes proposed in this paper. Here, we mention that a detailed analysis of the scheme introduced by Ernest et al. [6] is beyond the scope of this paper, and will be the topic of an upcoming paper.

To sum up, schemes in the present paper have the following properties:

(a) Approximate solutions for (1.1) and (1.2), generated by the scheme (3.2), converge to the unique entropy solution of (1.4) as long as $\mu(\Delta x)=\mathcal{O}\left(\Delta x^{2}\right)$. A scheme ( $\left.c f .(7.2)\right)$ has been formulated for (1.1) and (1.3) and the same techniques can be applied, mutatis mutandis, to prove convergence of approximate solutions to the unique entropy solution of (1.4). 
(b) Approximate solutions for (1.1) and (1.2) have been shown to converge to weak solutions of (1.4), when $\mu(\Delta x)=\mathcal{O}\left(\Delta x^{2}\right)$. Moreover, we show numerically that the limiting solutions generated by the schemes (3.2) and (7.2) contain nonclassical undercompressive shock waves.

The rest of the paper is organized as follows: In Section 2, we present the mathematical framework used in this paper. In particular, we have used a compensated compactness result in the spirit of Tartar [28] but the proof is based on div-curl lemma and does not rely on the Young measure. Section 3 introduces the fully-discrete finite difference scheme for (1.1) and (1.2). In Section 4, we derive a priori estimates for the approximate solutions and a detailed convergence analysis towards weak solutions of (1.4) has been discussed in Section 5. Convergence towards the unique entropy solution has been considered in Section 6, while a brief discussion on the results for diffusive-dispersive approximation (1.1) and (1.3) has been addressed in Section 7. Finally, numerical results are presented in Section 8 to illustrate the performance of the designed schemes.

\section{MATHEMATiCAL FRAMEWORK}

In this section, we list all the assumptions on the data for the problem (1.1), and present relevant mathematical tools to be used in the subsequent analysis. Throughout this paper we use the letters $C, K$ etc. to denote various generic constants independent of approximation parameters, which may change line to line, but the notation is kept unchanged so long as it does not impact the central idea.

The basic assumptions on the data of the problem (1.1) are as follows:

A.1 For the initial function $u_{0}: \mathbb{R} \mapsto \mathbb{R}$, we assume that

$$
u_{0} \in L^{2}(\mathbb{R}) \cap L^{\infty}(\mathbb{R}), \quad a \leq u_{0}(x) \leq b, \text { for a.e } x \in \mathbb{R} ;
$$

A.2 For the discontinuous coefficient $k: \mathbb{R} \mapsto \mathbb{R}$, we assume that

$$
k \in L^{\infty}(\mathbb{R}) \cap B V_{\text {loc }}(\mathbb{R}), \quad \alpha \leq k(x) \leq \beta, \text { for a.e } x \in \mathbb{R} ;
$$

A.3 Regarding the flux function $f:[\alpha, \beta] \times[a, b] \mapsto \mathbb{R}$, we assume that

$$
\begin{aligned}
& u \mapsto f(k, u) \in C^{2}([a, b]), \text { for all } k \in[\alpha, \beta], \\
& k \mapsto f(k, u) \in C^{1}([\alpha, \beta]), \text { for all } u \in[a, b] ;
\end{aligned}
$$

A.4 Furthermore, we assume that $u \mapsto f(k, u)$ is genuinely nonlinear a.e. in $\mathbb{R} \times[0, T]$, i.e., $f_{u u}(k(x), u) \neq 0$, for a.e. $u \in[a, b]$.

Remark 2.1. It is worth mentioning that Assumption A.4 is typically required in the compensated compactness framework. This condition also imposes a condition on the coefficient $k(x)$. In fact, it implies that $f(u)$ is genuinely nonlinear (i.e., $\left.f^{\prime \prime} \neq 0\right)$ and $|k(x)| \neq 0$, for a.e. $x \in \mathbb{R}$.

Next, we recapitulate the results required from the compensated compactness method due to Murat and Tartar $[24,28]$. For a nice overview of applications of the compensated compactness method to hyperbolic conservation laws, we refer to Chen $[3]$. Let $\mathcal{M}(\mathbb{R})$ denote the space of bounded Radon measures on $\mathbb{R}$ and

$$
C_{0}(\mathbb{R})=\left\{\psi \in C(\mathbb{R}) \mid \lim _{|x| \rightarrow \infty} \psi(x)=0\right\} .
$$

If $\mu \in \mathcal{M}(\mathbb{R})$, then

$$
\langle\mu, \psi\rangle=\int_{\mathbb{R}} \psi \mathrm{d} \mu, \quad \text { for all } \quad \psi \in C_{0}(\mathbb{R}) .
$$


Recall that $\mu \in \mathcal{M}(\mathbb{R})$ if and only if $|\langle\mu, \psi\rangle| \leq C\|\psi\|_{L^{\infty}(\mathbb{R})}$ for all $\psi \in C_{0}(\mathbb{R})$. We define the norm

$$
\|\mu\|_{\mathcal{M}(\mathbb{R})}:=\sup \left\{|\langle\mu, \psi\rangle|: \psi \in C_{0}(\mathbb{R}),\|\psi\|_{L^{\infty}(\mathbb{R})} \leq 1\right\}
$$

The space $\left(\mathcal{M}(\mathbb{R}),\|\cdot\|_{\mathcal{M}(\mathbb{R})}\right)$ is a Banach space and it is isometrically isomorphic to the dual space of $\left(C_{0}(\mathbb{R}),\|\cdot\|_{L^{\infty}(\mathbb{R})}\right)$. Furthermore, we define the space of probablity measures

$$
\operatorname{Prob}(\mathbb{R}):=\left\{\mu \in \mathcal{M}(\mathbb{R}): \mu \text { is nonnegative and }\|\mu\|_{\mathcal{M}(\mathbb{R})}=1\right\}
$$

Before we state the compensated compactness theorem, we shall recall the celebrated div-curl lemma [24].

Lemma 2.2 (div-curl lemma). Let $\Omega$ be a bounded open subset of $\mathbb{R}^{2}$. Suppose

$$
u_{\Delta x}^{1} \rightarrow \bar{u}^{1}, \quad u_{\Delta x}^{2} \rightarrow \bar{u}^{2}, \quad v_{\Delta x}^{1} \rightarrow \bar{v}^{1}, \text { and } v_{\Delta x}^{2} \rightarrow \bar{v}^{2},
$$

in $L^{2}(\Omega)$ as $\Delta x \quad \downarrow \quad 0$. Furthermore, assume that the two sequences $\left\{\operatorname{div}\left(u_{\Delta x}^{1}, u_{\Delta x}^{2}\right)\right\}_{\Delta x>0}$ and $\left\{\operatorname{curl}\left(v_{\Delta x}^{1}, v_{\Delta x}^{2}\right)\right\}_{\Delta x>0}$ lie in a (common) compact subset of $H_{\mathrm{loc}}^{-1}(\Omega)$, where $\operatorname{div}\left(u_{\Delta x}^{1}, u_{\Delta x}^{2}\right)=\partial_{x_{1}} u_{\Delta x}^{1}+\partial_{x_{2}} u_{\Delta x}^{2}$ and $\operatorname{curl}\left(v_{\Delta x}^{1}, v_{\Delta x}^{2}\right)=\partial_{x_{1}} v_{\Delta x}^{2}-\partial_{x_{2}} v_{\Delta x}^{1}$. Then along a subsequence

$$
\left(u_{\Delta x}^{1}, u_{\Delta x}^{2}\right) \cdot\left(v_{\Delta x}^{1}, v_{\Delta x}^{2}\right) \mapsto\left(\bar{u}^{1}, \bar{u}^{2}\right) \cdot\left(\bar{v}^{1}, \bar{v}^{2}\right) \text {, in } \mathcal{D}^{\prime}(\Omega) \text {, as } \Delta x \downarrow 0 .
$$

Suitably modified for our purpose, we shall use the following compensated compactness result. For a proof, we refer to the paper by Karlsen and Towers ([13], Lem. 3.2).

Theorem 2.3. Assume that A.2, A.3 and A.4 hold. Let $\Omega \subset \mathbb{R} \times[0, T]$ be a bounded open set, and assume that $\left\{u_{\Delta x}\right\}$ is a sequence of uniformly bounded functions such that $\left|u_{\Delta x}\right| \leq M$, for all $\Delta x$. Set

$$
\begin{aligned}
\left(\eta_{1}(s), q_{1}(k, s)\right) & =(s-c, f(k, s)-f(k, c)), \\
\left(\eta_{2}(k, s), q_{2}(k, s)\right) & =\left(f(k, s)-f(k, c), \int_{c}^{s}\left(f_{s}(k, \theta)\right)^{2} \mathrm{~d} \theta\right),
\end{aligned}
$$

where $c$ is an arbitrary constant. If the two sequences

$$
\left\{\eta_{1}\left(u_{\Delta x}\right)_{t}+q_{1}\left(k(x), u_{\Delta x}\right)_{x}\right\}_{\Delta x>0}, \quad \text { and } \quad\left\{\eta_{2}\left(k(x), u_{\Delta x}\right)_{t}+q_{2}\left(k(x), u_{\Delta x}\right)_{x}\right\}_{\Delta x>0}
$$

belong to a compact subset of $H_{\mathrm{loc}}^{-1}(\Omega)$, then there exists a subsequence of $\left\{u_{\Delta x}\right\}_{\Delta x>0}$ that converges a.e. to a function $u \in L^{\infty}(\Omega)$.

We remark that, a feature of the compensated compactness result above is that it avoids the use of the Young measure by following an approach developed by Chen and $\mathrm{Lu}[3,23]$ for the standard scalar conservation law. This is preferable as the fundamental theorem of Young measures applies most easily to functions that are continuous in all variables.

The following compactness interpolation result (known as Murat's lemma [24]) is useful in obtaining the $H_{\text {loc }}^{-1}$ compactness needed in Theorem 2.3.

Lemma 2.4. Let $\Omega$ be a bounded open subset of $\mathbb{R}^{2}$. Suppose that the sequence $\left\{\mathcal{L}_{\Delta x}\right\}_{\Delta x>0}$ of distributions is bounded in $W^{-1, \infty}(\Omega)$. Suppose also that

$$
\mathcal{L}_{\Delta x}=\mathcal{L}_{1, \Delta x}+\mathcal{L}_{2, \Delta x},
$$

where $\left\{\mathcal{L}_{1, \Delta x}\right\}_{\Delta x>0}$ is in a compact subset of $H_{\text {loc }}^{-1}(\Omega)$ and $\left\{\mathcal{L}_{2, \Delta x}\right\}_{\Delta x>0}$ is in a bounded subset of $\mathcal{M}_{\text {loc }}(\Omega)$. Then $\left\{\mathcal{L}_{\Delta x}\right\}_{\Delta x>0}$ is in a compact subset of $H_{\mathrm{loc}}^{-1}(\Omega)$. 


\section{A Fully-DiscRete FINITE DIFFERENCE SCHEME}

We begin by introducing some notation needed to define the fully-discrete finite difference scheme. Throughout this paper, we reserve $\Delta x, \Delta t$ to denote small positive numbers that represent the spatial and temporal discretizations parameter of the numerical scheme. Given $\Delta x>0$, we set $x_{j}=j \Delta x$ for $j \in \mathbb{Z}$, to denote the spatial mesh points. Similarly, we set $t^{n}=n \Delta t$ for $n=0,1, \ldots, N$, where $N \Delta t=T$ for some fixed time horizon $T>0$. Moreover, for any function $u=u(x, t)$ admitting point values, we write $u_{j}^{n}=u\left(x_{j}, t^{n}\right)$. Furthermore, let us introduce the spatial and spatial-temporal grid cells

$$
I_{j}=\left[x_{j-1 / 2}, x_{j+1 / 2}\right), \quad I_{j}^{n}=\left[x_{j-1 / 2}, x_{j+1 / 2}\right) \times\left[t^{n}, t^{n+1}\right) .
$$

where $x_{j \pm 1 / 2}=x_{j} \pm \Delta x / 2$. Let $D_{ \pm}$denote the discrete forward and backward differences in space, i.e.,

$$
D_{ \pm} u_{j}= \pm \frac{u_{j \pm 1}-u_{j}}{\Delta x}
$$

The discrete Leibnitz rule is given by

$$
D_{ \pm}\left(u_{j} v_{j}\right)=u_{j} D_{ \pm} v_{j}+v_{j \pm 1} D_{ \pm} u_{j}
$$

while the summation-by-parts formula is given by

$$
\sum_{j \in \mathbb{Z}} u_{j} D_{ \pm} v_{j}=-\sum_{j \in \mathbb{Z}} v_{j} D_{\mp} u_{j}
$$

Furthermore, for any $C^{2}$ function $f$, using the Taylor expansion on the sequence $f\left(u_{j}\right)$ we obtain

$$
D_{ \pm} f\left(u_{j}\right)=f^{\prime}\left(u_{j}\right) D_{ \pm} u_{j} \pm \frac{\Delta x}{2} f^{\prime \prime}\left(\xi_{j \pm \frac{1}{2}}\right)\left(D_{ \pm} u_{j}\right)^{2}
$$

for some $\xi_{j \pm \frac{1}{2}}$ between $u_{j \pm 1}$ and $u_{j}$. In other words, the discrete chain rule is accurate up to an error term of order $\Delta x\left(D_{ \pm} u_{j}\right)^{2}$.

Finally, let $D_{ \pm}^{t}$ denote the discrete forward and backward difference operator in the time, i.e.,

$$
D_{ \pm}^{t} u_{j}^{n}=\mp \frac{u_{j}^{n \pm 1}-u_{j}^{n}}{\Delta t}
$$

The following identity is readily verified:

$$
u_{j}^{n} D_{+}^{t} u_{j}^{n}=\frac{1}{2} D_{+}^{t}\left(u_{j}^{n}\right)^{2}-\frac{\Delta t}{2}\left(D_{+}^{t} u_{j}^{n}\right)^{2} .
$$

We propose the following fully-discrete (in space and time) finite difference scheme approximating the limiting solutions generated by equation (1.1)-(1.2)

$$
\begin{aligned}
D_{+}^{t} u_{j}^{n}+D_{-} h_{j+\frac{1}{2}}^{n} & =\beta \Delta x D_{+} D_{-} u_{j}^{n}+\gamma \mu(\Delta x) D_{+}^{t} D_{+} D_{-} u_{j}^{n}, \quad j \in \mathbb{Z}, n \in \mathbb{N}_{0}, \\
u_{j}^{0} & =\frac{1}{\Delta x} \int_{x_{j-\frac{1}{2}}}^{x_{j+\frac{1}{2}}} u_{0}(\theta) \mathrm{d} \theta, \quad j \in \mathbb{Z},
\end{aligned}
$$

where $\beta, \gamma>0$ are fixed parameters, and $\mu(\Delta x) \mapsto 0$ as $\Delta x \mapsto 0$. More specifically, we will either use $\mu(\Delta x)=$ $\mathcal{O}\left(\Delta x^{2}\right)$ or $\mu(\Delta x)=\mathcal{O}\left(\Delta x^{2}\right)$ depending on the quest for the convergence of approximate solution $u_{\Delta x}$ towards a weak solution or the entropy solution, respectively. 
Remark 3.1. Here we used the notation $\mathcal{O}(\Delta x)$ to denote quantities that depend on $\Delta x$ and are bounded above by $C \Delta x$, where $C$ is a constant independent of $\Delta x$. Likewise, we used the notation $\mu(\Delta x)=\mathcal{O}(\Delta x)$ to denote quantities that depend on $\Delta x$ and are bounded above by $C \Delta x^{\alpha}$, where $C$ is a constant independent of $\Delta x$ and $\alpha>1$.

The numerical flux corresponding to the flux function $k(x) f(u)$ is given by

$$
h_{j+\frac{1}{2}}^{n}=k_{j+\frac{1}{2}} \hat{f}_{j+\frac{1}{2}}^{n}, \quad \text { with } k_{j+\frac{1}{2}}=\frac{1}{\Delta x} \int_{x_{j}}^{x_{j+1}} k(x) \mathrm{d} x,
$$

where $\hat{f}_{j+\frac{1}{2}}^{n}:=\hat{f}^{n}\left(u_{j}, u_{j+1}\right)$ is based on a two-point monotone numerical flux, i.e., non-decreasing with respect to the first argument and non-increasing with respect to the second argument, and consistent with the actual flux, i.e., $\hat{f}(u, u)=f(u)$. Moreover, in order to maintain monotonicity of the scheme (3.2) without the higher order terms (corresponds to $\beta=\gamma=0$ ), the arguments of the numerical flux are transposed when the coefficient $k$ is negative. More specifically, we choose

$$
\hat{f}_{j+\frac{1}{2}}^{n}= \begin{cases}\hat{f}\left(u_{j}^{n}, u_{j+1}^{n}\right), & \text { if } k_{j+\frac{1}{2}} \geq 0 \\ \hat{f}\left(u_{j+1}^{n}, u_{j}^{n}\right), & \text { if } k_{j+\frac{1}{2}}<0 .\end{cases}
$$

Summing up, the numerical flux $h_{j+\frac{1}{2}}^{n}$ is given by

$$
h_{j+\frac{1}{2}}^{n}= \begin{cases}k_{j+\frac{1}{2}} \hat{f}\left(u_{j}^{n}, u_{j+1}^{n}\right), & \text { if } k_{j+\frac{1}{2}} \geq 0 \\ k_{j+\frac{1}{2}} \hat{f}\left(u_{j+1}^{n}, u_{j}^{n}\right), & \text { if } k_{j+\frac{1}{2}}<0 .\end{cases}
$$

In particular, we focus on Engquist-Osher (EO) numerical flux given by

$$
\hat{f}(u, v)=\frac{1}{2}(f(u)+f(v))-\frac{1}{2} \int_{u}^{v}\left|f^{\prime}(s)\right| \mathrm{d} s .
$$

Remark 3.2. We have chosen to analyse the scheme (3.2) and (3.3) with EO flux because of its apparent simplicity. One can, however, adopt the method of proof developed in this paper and obtain similar results for other schemes (e.g., all monotone schemes).

To this end, observe that the EO flux given by (3.4) is Lipschitz continuous. In fact, for $f \in C^{1}$, it has continuous partial derivatives satisfying

$$
f_{-}^{\prime}(v)=\hat{f}_{v}(v, u) \leq 0 \leq \hat{f}_{u}(v, u)=f_{+}^{\prime}(u)
$$

using the conventional notations that $a_{-}=\min (a, 0)$ and $a_{+}=\max (a, 0)$. It is also clear that $\left\|f^{\prime}\right\|_{\infty}$ serves as a Lipschitz constant for EO flux.

For a given initial data $u_{0}$, we define the initial grid function $\left\{u_{j}^{0}\right\}_{j \in \mathbb{Z}}$ by (3.3). Moreover, for the sequence $\left\{u_{j}^{n}\right\}_{j \in \mathbb{Z}, n \in \mathbb{N}_{0}}$, we associate the function $u_{\Delta x}$ defined by

$$
u_{\Delta x}(x, t)=\sum_{j \in \mathbb{Z}, n \geq 0} u_{j}^{n} \mathbb{1}_{I_{j}^{n}}(x, t),
$$

where $\mathbb{1}_{A}$ denotes the characteristic function of the set $A$. Similarly, for the coefficient $k$ approximated at each cell boundary, we associate the function $k_{\Delta x}$ defined by

$$
k_{\Delta x}(x)=\sum_{j \in \mathbb{Z}} k_{j+\frac{1}{2}} \mathbb{1}_{I_{j+\frac{1}{2}}}(x)
$$


Note that $k$ and $u$ are discretized on grids that are staggered with respect to each other. This indeed results in a reduction in complexity, compared with the approach where two discretizations are aligned.

Throughout this paper, we use the notation $u_{\Delta x}$ to denote the functions associated with the sequence $\left\{u_{j}^{n}\right\}_{j \in \mathbb{Z}, n \in \mathbb{N}_{0}}$. For later use, recall that the discrete $\ell^{\infty}(\mathbb{R}), \ell^{1}(\mathbb{R})$ and $\ell^{2}(\mathbb{R})$ norms, and BV semi-norm for a lattice function $u_{\Delta x}$ are defined respectively as

$$
\begin{aligned}
& \left\|u_{\Delta x}\left(\cdot, t^{n}\right)\right\|_{\ell^{\infty}(\mathbb{R})}=\sup _{j \in \mathbb{Z}}\left|u_{j}^{n}\right|, \quad\left\|u_{\Delta x}\left(\cdot, t^{n}\right)\right\|_{\ell^{1}(\mathbb{R})}=\Delta x \sum_{j \in \mathbb{Z}}\left|u_{j}^{n}\right|, \\
& \left\|u_{\Delta x}\left(\cdot, t^{n}\right)\right\|_{\ell^{2}(\mathbb{R})}=\sqrt{\Delta x \sum_{j \in \mathbb{Z}}\left|u_{j}^{n}\right|^{2}}, \quad\left|u_{\Delta x}\left(\cdot, t^{n}\right)\right|_{B V}=\sum_{j \in \mathbb{Z}}\left|u_{j+1}^{n}-u_{j}^{n}\right| .
\end{aligned}
$$

For the sake of simplified notations, unless specified, we shall use the notation $\|\cdot\|$ to denote the discrete $\ell^{2}(\mathbb{R})$ norm.

\section{A PRIORI ESTIMATes}

This section is devoted to the derivation of a priori estimates which turns out to be useful to prove "strong compactness" of the approximate solution $u_{\Delta x}$. To begin with, following Coclite et al. [4], we assume that the approximate solutions generated by the scheme are uniformly bounded, i.e., $u_{\Delta x} \in L^{\infty}(\mathbb{R} \times[0, T])$. In other words, we assume that

B.1 For almost every $(x, t) \in \mathbb{R} \times[0, T], a^{\prime} \leq u_{\Delta x} \leq b^{\prime}$, for some fixed constants $a^{\prime}, b^{\prime} \in \mathbb{R}$;

Remark 4.1. It is worth mentioning that the above assumption on the approximate solutions is the manifestation of the specific structure of the flux function (depends explicitly on space variable). In fact, to obtain $L^{2}$ bound on the solution, one requires a priori $L^{\infty}$ bound on the solution ( $c f$. (4.4)). This assumption can be toppled by replacing the "space dependent flux function" to a flux function which depends explicitly only on the solution. In such a scenario, one can use $L^{p}$ framework of the compensated compactness result [5], to reproduce all the results in this paper.

To proceed further, we first collect all the available estimates on the approximate solutions in the following lemma.

Lemma 4.2. Let $u_{\Delta x}$ be a sequence of approximations generated by the scheme (3.2). Moreover, assume that the initial data $u_{0}$ lies in $L^{2}(\mathbb{R})$. Then the following estimate holds

$$
\begin{aligned}
\frac{1}{2} D_{+}^{t}\left\|u^{n}\right\|^{2}+\left(\frac{\gamma \mu(\Delta x)}{2}+\frac{\beta \Delta x^{2}}{2}\right) D_{+}^{t}\left\|D_{-} u^{n}\right\|^{2} & +\delta \Delta x\left\|D_{-} u^{n}\right\|^{2} \\
& +\delta \Delta x\left\|D_{+}^{t} u^{n}\right\|^{2}+\delta \gamma \Delta x \mu(\Delta x)\left\|D_{+}^{t} D_{-} u^{n}\right\|^{2} \leq C,
\end{aligned}
$$

provided $\Delta t$ and $\Delta x$ satisfies the following CFL condition

$$
\max \left\{2 \max \left\{\|f\|_{\infty},\left\|f^{\prime}\right\|_{\infty},\|k\|_{\infty}\right\}+\frac{\lambda}{2}, \frac{\lambda}{2}\left(1+\frac{\beta \Delta x^{2}}{\gamma \mu(\Delta x)}\right)\right\} \leq \min (1-\delta, \beta-\delta),
$$

with $\delta \in(0, \min (1, \beta))$. Here $\lambda=\Delta t / \Delta x$ and the constant $C>0$ is independent of $\Delta x$. 
In particular, the estimate (4.1) guarantees following space-time estimates:

$$
\begin{array}{r}
\forall n \in \mathbb{N}, \quad \Delta x \sum_{j}\left(u_{j}^{n}\right)^{2} \leq C, \\
\Delta x^{2} \Delta t \sum_{j} \sum_{n}\left(D_{-} u_{j}^{n}\right)^{2} \leq C, \\
\Delta x^{2} \Delta t \sum_{j} \sum_{n}\left(D_{+}^{t} u_{j}^{n}\right)^{2} \leq C, \\
\Delta t \Delta x^{2} \mu(\Delta x) \sum_{j} \sum_{n}\left(D_{+}^{t} D_{-} u_{j}^{n}\right)^{2} \leq C .
\end{array}
$$

Remark 4.3. In light of the CFL condition (4.2), we want to emphasize that if $\mu(\Delta x)=\mathcal{O}\left(\Delta x^{2}\right)$ (required to prove convergence towards a weak solution, $c f$. Thm. 5.8), then we need $\zeta:=\frac{\lambda(\Delta x)^{2}}{\mu(\Delta x)}=\frac{\Delta t}{\Delta x}$ to be kept fixed. On the other hand, if $\mu(\Delta x)=\mathcal{O}\left(\Delta x^{2}\right)$ (required to prove convergence towards the entropy solution, $c f$. Thm. 6.4), then we need $\zeta=\frac{\Delta t}{(\Delta x)^{1+\alpha}}$, with $\alpha>0$, to be kept fixed. To sum up, we need a stronger CFL condition to prove convergence of approximate solutions towards the unique entropy solution of (1.1). To this end, we mention that in the subsequent analysis the CFL condition (4.2) is always assumed to hold.

Proof. To start with, we multiply the scheme (3.2) by $\Delta x u_{j}^{n}$ and subsequently sum over $j \in \mathbb{Z}$. Then, using summation-by-parts formula and the identity (3.1), we obtain

$$
\begin{aligned}
\frac{1}{2} D_{+}^{t} \sum_{j} \Delta x\left(u_{j}^{n}\right)^{2}-\frac{\Delta t}{2} \sum_{j} \Delta x\left(D_{+}^{t} u_{j}^{n}\right)^{2}+\underbrace{\Delta x \sum_{j} u_{j}^{n} D_{-} h_{j+\frac{1}{2}}^{n}}_{\mathcal{I}_{\Delta x}(f)} \\
=-\beta \Delta x \sum_{j} \Delta x\left|D_{-} u_{j}\right|^{2}-\gamma \mu(\Delta x) \Delta x \sum_{j} D_{-} u_{j}^{n} D_{+}^{t}\left(D_{-} u_{j}^{n}\right) .
\end{aligned}
$$

Note that, the identity (3.1) also implies that

$$
\mu(\Delta x) \Delta x \sum_{j} D_{-} u_{j}^{n} D_{+}^{t}\left(D_{-} u_{j}^{n}\right)=\frac{\Delta x \mu(\Delta x)}{2} \sum_{j} D_{+}^{t}\left(D_{-} u_{j}^{n}\right)^{2}-\frac{\Delta x \mu(\Delta x) \Delta t}{2} \sum_{j}\left(D_{+}^{t} D_{-} u_{j}^{n}\right)^{2} .
$$

Next, we move on to estimate the term $\mathcal{I}_{\Delta x}(f)$. Using summation-by-parts formula, we obtain

$$
\begin{aligned}
-\mathcal{I}_{\Delta x}(f) & =-\sum_{j} \Delta x u_{j}^{n} D_{+} h_{j-\frac{1}{2}}^{n}=\sum_{j}\left(u_{j}^{n}-u_{j-1}^{n}\right) k_{j-\frac{1}{2}} \hat{f}_{j-\frac{1}{2}}^{n} \\
& =\sum_{j} k_{j-\frac{1}{2}}\left[\left(u_{j}^{n}-u_{j-1}^{n}\right) \hat{f}_{j-\frac{1}{2}}^{n}-\left(F\left(u_{j}^{n}\right)-F\left(u_{j-1}^{n}\right)\right)\right]+\sum_{j} k_{j-\frac{1}{2}}\left(F\left(u_{j}^{n}\right)-F\left(u_{j-1}^{n}\right)\right) \\
& =\sum_{j} \underbrace{k_{j-\frac{1}{2}}\left[\left(u_{j}^{n}-u_{j-1}^{n}\right) \hat{f}_{j-\frac{1}{2}}^{n}-\left(F\left(u_{j}^{n}\right)-F\left(u_{j-1}^{n}\right)\right)\right]}_{\mathcal{E}_{j, n}(f)}-\sum_{j} F\left(u_{j}^{n}\right)\left(k_{j+\frac{1}{2}}-k_{j-\frac{1}{2}}\right),
\end{aligned}
$$

where $F$ is the primitive of $f$, i.e., $F^{\prime}=f$. A first order Taylor's expansion together with the monotonicity of the numerical flux function $\hat{f}\left(u_{j}^{n}, u_{j+1}^{n}\right)$ gives us an estimate of $\mathcal{E}_{j, n}(f)$. To see this, notice that

$$
\mathcal{E}_{j, n}(f)=k_{j-\frac{1}{2}}\left(u_{j}^{n}-u_{j-1}^{n}\right)\left(\hat{f}_{j-\frac{1}{2}}^{n}-f\left(u_{j-\frac{1}{2}}^{n}\right)\right) .
$$


where $u_{j-\frac{1}{2}}^{n}$ lies in between $u_{j}^{n}$ and $u_{j-1}^{n}$. To proceed further, we consider the following two cases:

Case 1. Assume that $k_{j-\frac{1}{2}} \geq 0$, then by the definition of numerical flux $\hat{f}_{j-\frac{1}{2}}^{n}=\hat{f}\left(u_{j-1}^{n}, u_{j}^{n}\right)$. If $u_{j}^{n} \leq u_{j-\frac{1}{2}}^{n} \leq$ $u_{j-1}^{n}$, then

$$
\hat{f}_{j-\frac{1}{2}}^{n} \geq \hat{f}\left(u_{j-\frac{1}{2}}^{n}, u_{j}^{n}\right) \geq \hat{f}\left(u_{j-\frac{1}{2}}^{n}, u_{j-\frac{1}{2}}^{n}\right)=f\left(u_{j-\frac{1}{2}}^{n}\right) .
$$

On the other hand, if $u_{j-1}^{n} \leq u_{j-\frac{1}{2}}^{n} \leq u_{j}^{n}$, then

$$
\hat{f}_{j-\frac{1}{2}}^{n} \leq \hat{f}\left(u_{j-\frac{1}{2}}^{n}, u_{j}^{n}\right) \leq \hat{f}\left(u_{j-\frac{1}{2}}^{n}, u_{j-\frac{1}{2}}^{n}\right)=f\left(u_{j-\frac{1}{2}}^{n}\right) .
$$

Thus, in any case we have $\mathcal{E}_{j, n}(f) \leq 0$.

Case 2. Assume that $k_{j-\frac{1}{2}}<0$, i.e., $\hat{f}_{j-\frac{1}{2}}^{n}=\hat{f}\left(u_{j}^{n}, u_{j-1}^{n}\right)$. If $u_{j}^{n} \leq u_{j-\frac{1}{2}}^{n} \leq u_{j-1}^{n}$, then

$$
\hat{f}_{j-\frac{1}{2}}^{n} \leq \hat{f}\left(u_{j-\frac{1}{2}}^{n}, u_{j-1}^{n}\right) \leq \hat{f}\left(u_{j-\frac{1}{2}}^{n}, u_{j-\frac{1}{2}}^{n}\right)=f\left(u_{j-\frac{1}{2}}^{n}\right) .
$$

On the other hand, if $u_{j-1}^{n} \leq u_{j-\frac{1}{2}}^{n} \leq u_{j}^{n}$, then

$$
\hat{f}_{j-\frac{1}{2}}^{n} \geq \hat{f}\left(u_{j-\frac{1}{2}}^{n}, u_{j-1}^{n}\right) \geq \hat{f}\left(u_{j-\frac{1}{2}}^{n}, u_{j-\frac{1}{2}}^{n}\right)=f\left(u_{j-\frac{1}{2}}^{n}\right) .
$$

Therefore, in this case also, we have $\mathcal{E}_{j, n}(f) \leq 0$. Having this in mind and making use of the assumption B.1, we conclude that

$$
-\mathcal{I}_{\Delta x}(f) \leq\left|-\sum_{j}\left(k_{j-\frac{1}{2}}-k_{j+\frac{1}{2}}\right) F\left(u_{j}^{n}\right)\right| \leq \mathrm{BV}(k) \max _{j}\left|F\left(u_{j}^{n}\right)\right| \leq C,
$$

where $C$ is a constant independent of $\Delta x$. Finally, combining all the above estimates, we obtain

$$
\frac{1}{2} D_{+}^{t}\left\|u^{n}\right\|^{2}-\frac{\Delta t}{2}\left\|D_{+}^{t} u^{n}\right\|^{2}+\frac{\gamma \mu(\Delta x)}{2} D_{+}^{t}\left\|D_{-} u^{n}\right\|^{2}-\frac{\gamma \mu(\Delta x) \Delta t}{2}\left\|D_{+}^{t} D_{-} u^{n}\right\|^{2}+\beta \Delta x\left\|D_{-} u^{n}\right\|^{2} \leq C .
$$

Next, we multiply the scheme (3.2) by $\Delta x^{2} D_{+}^{t} u_{j}^{n}$ and sum over $j \in \mathbb{Z}$ to obtain,

$$
\Delta x^{2} \sum_{j}\left(D_{+}^{t} u_{j}^{n}\right)^{2}+\Delta x^{2} \sum_{j} D_{-} h_{j+\frac{1}{2}}^{n} D_{+}^{t} u_{j}^{n}=-\beta(\Delta x)^{3} \sum_{j} D_{-} u_{j}^{n} D_{+}^{t}\left(D_{-} u_{j}^{n}\right)-\gamma \mu(\Delta x) \Delta x^{2} \sum_{j}\left(D_{+}^{t} D_{-} u_{j}^{n}\right)^{2} .
$$

Again, use of the identity (3.1) reveals that

$$
\beta(\Delta x)^{3} \sum_{j} D_{-} u_{j}^{n} D_{+}^{t}\left(D_{-} u_{j}^{n}\right)=\frac{\beta \Delta x^{3}}{2} \sum_{j} D_{+}^{t}\left(D_{-} u_{j}^{n}\right)^{2}-\frac{\beta \Delta x^{3} \Delta t}{2} \sum_{j}\left(D_{+}^{t} D_{-} u_{j}^{n}\right)^{2} .
$$

Next, considering the term which involves flux function, we see that

$$
\begin{aligned}
& \Delta x^{2} \sum_{j} D_{-} h_{j+\frac{1}{2}} D_{+}^{t} u_{j}^{n}=\Delta x \sum_{j}\left(k_{j+\frac{1}{2}} \hat{f}_{j+\frac{1}{2}}^{n}-k_{j-\frac{1}{2}} \hat{f}_{j-\frac{1}{2}}^{n}\right) D_{+}^{t} u_{j}^{n} \\
& \quad=\Delta x \sum_{j}\left(k_{j+\frac{1}{2}}-k_{j-\frac{1}{2}}\right) \hat{f}_{j+\frac{1}{2}}^{n} D_{+}^{t} u_{j}^{n}+\Delta x \sum_{j} k_{j-\frac{1}{2}}\left(\hat{f}_{j+\frac{1}{2}}^{n}-\hat{f}_{j-\frac{1}{2}}^{n}\right) D_{+}^{t} u_{j}^{n} .
\end{aligned}
$$

Recall that we have chosen to work with a specific monotone flux, i.e., Engquist Osher flux. Since EO flux is Lipschitz continuous with Lipschitz constant $\left\|f^{\prime}\right\|_{\infty}(c f$. (3.5)), we conclude that

$$
\operatorname{supsup}_{j}\left|\hat{f}_{j+\frac{1}{2}}^{n}\right| \leq\|f\|_{\infty} \text {, and }\left|\hat{f}_{j+\frac{1}{2}}^{n}-\hat{f}_{j-\frac{1}{2}}^{n}\right| \leq\left\|f^{\prime}\right\|_{\infty}\left(\left|u_{j}^{n}-u_{j-1}^{n}\right|+\left|u_{j+1}^{n}-u_{j}^{n}\right|\right) .
$$


An application of Young's inequality: for any two real numbers $a$ and $b$ and for every $\varepsilon>0$

$$
a b \leq \varepsilon a^{2}+\frac{b^{2}}{4 \varepsilon}
$$

leads to the estimates

$$
\begin{aligned}
\Delta x \sum_{j}\left(k_{j+\frac{1}{2}}-k_{j-\frac{1}{2}}\right) \hat{f}_{j+\frac{1}{2}}^{n} D_{+}^{t} u_{j}^{n} & \leq \Delta x\|f\|_{\infty} \sum_{j}\left|\left(k_{j+\frac{1}{2}}-k_{j-\frac{1}{2}}\right)\right|\left|D_{+}^{t} u_{j}^{n}\right| \\
& \leq \varepsilon \Delta x\|f\|_{\infty}\left\|D_{+}^{t} u^{n}\right\|^{2}+\frac{\|f\|_{\infty} \Delta x}{4 \varepsilon}\left\|D_{+} k\right\|^{2}
\end{aligned}
$$

and

$$
\begin{aligned}
\Delta x \sum_{j} k_{j-\frac{1}{2}}\left(\hat{f}_{j+\frac{1}{2}}^{n}-\hat{f}_{j-\frac{1}{2}}^{n}\right) D_{+}^{t} u_{j}^{n} & \leq \Delta x^{2}\left\|f^{\prime}\right\|_{\infty}\|k\|_{\infty} \sum_{j}\left(\left|D_{-} u_{j}^{n}\right|+\left|D_{-} u_{j+1}^{n}\right|\right)\left|D_{+}^{t} u_{j}^{n}\right| \\
& \leq 2 \varepsilon_{1} \Delta x\left\|f^{\prime}\right\|_{\infty}\|k\|_{\infty}\left\|D_{+}^{t} u^{n}\right\|^{2}+\frac{\left\|f^{\prime}\right\|_{\infty}\|k\|_{\infty} \Delta x}{2 \varepsilon_{1}}\left\|D_{-} u^{n}\right\|^{2} .
\end{aligned}
$$

For notational simplification, we define $M:=\max \left\{\|f\|_{\infty},\left\|f^{\prime}\right\|_{\infty},\|k\|_{\infty}\right\}$. Combining all above estimates, we arrive

$$
\begin{aligned}
\Delta x\left\|D_{+}^{t} u^{n}\right\|^{2}+\gamma \Delta x \mu(\Delta x) & \left\|D_{+}^{t} D_{-} u^{n}\right\|^{2}+\frac{\beta \Delta x^{2}}{2} D_{+}^{t}\left\|D_{-} u^{n}\right\|^{2} \\
-\frac{\beta \Delta x^{2} \Delta t}{2}\left\|D_{+}^{t} D_{-} u^{n}\right\|^{2} & \leq \varepsilon \Delta x M\left\|D_{+}^{t} u^{n}\right\|^{2}+\frac{M \Delta x}{4 \varepsilon}\left\|D_{+} k\right\|^{2} \\
& +2 \varepsilon_{1} \Delta x M^{2}\left\|D_{+}^{t} u^{n}\right\|^{2}+\frac{M^{2} \Delta x}{2 \varepsilon_{1}}\left\|D_{-} u^{n}\right\|^{2} .
\end{aligned}
$$

Finally, adding (4.5) and (4.7) yields

$$
\begin{aligned}
\frac{1}{2} D_{+}^{t}\left\|u^{n}\right\|^{2}+\left(\frac{\gamma \mu(\Delta x)}{2}+\frac{\beta \Delta x^{2}}{2}\right) D_{+}^{t} & \left\|D_{-} u^{n}\right\|^{2}+\Delta x\left(\beta-\frac{M^{2}}{2 \varepsilon_{1}}\right)\left\|D_{-} u^{n}\right\|^{2} \\
+ & \Delta x\left(1-M \varepsilon-2 M^{2} \varepsilon_{1}-\frac{\lambda}{2}\right)\left\|D_{+}^{t} u^{n}\right\|^{2} \\
& +\gamma \Delta x \mu(\Delta x)\left(1-\frac{\lambda}{2}-\frac{\beta \lambda \Delta x^{2}}{2 \gamma \mu(\Delta x)}\right)\left\|D_{+}^{t} D_{-} u^{n}\right\|^{2} \leq C,
\end{aligned}
$$

where $\lambda=\frac{\Delta t}{\Delta x}$.

We must now use the CFL condition (4.2) to conclude that for some $\delta \in(0,1)$

$$
1-\frac{\lambda}{2}-\frac{\beta \lambda \Delta x^{2}}{2 \gamma \mu(\Delta x)}>\delta, \beta-\frac{M^{2}}{2 \varepsilon_{1}}>\delta, \text { and } 1-M \varepsilon-2 M^{2} \varepsilon_{1}-\frac{\lambda}{2}>\delta .
$$

Note that $\beta>\delta$ must hold. Furthermore, choosing $\varepsilon=1, \varepsilon_{1}=0.5$, the third contraint in (4.9) can be written as

$$
M+M^{2}+\frac{\lambda}{2}<1-\delta
$$

which would require $M<1$. Assuming $M$ is small enough (this can be done upto rescaling) to ensure the existence of a $\delta \in(0,1)$, such that $2 M+\frac{\lambda}{2}<\min (1-\delta, \beta-\delta)$. This in turn would imply

$$
\begin{gathered}
M^{2}+M+\frac{\lambda}{2}<2 M+\frac{\lambda}{2}<1-\delta, \\
\beta-M^{2}>\beta-2 M>\delta .
\end{gathered}
$$


In other words, we get the last two conditions of (4.9). Thus, the CFL condition (4.2) ensures all the conditions of (4.9) are satisfied. Consequently, we see that estimate (4.1) holds.

In order to prove estimates $(4.3 \mathrm{a}),(4.3 \mathrm{~b}),(4.3 \mathrm{c})$, and $(4.3 \mathrm{~d})$, we multiply the inequality (4.8) by $\Delta t$ and subsequently sum over all $n=0,1, \ldots, N-1$ to reach

$$
\begin{aligned}
\frac{1}{2}\left\|u^{N}\right\|^{2}+\left(\frac{\gamma \mu(\Delta x)}{2}\right. & \left.+\frac{\beta \Delta x^{2}}{2}\right)\left\|D_{-} u^{N}\right\|^{2}+\delta \Delta x \Delta t \sum_{n}\left\|D_{-} u^{n}\right\|^{2} \\
& +\delta \Delta x \Delta t \sum_{n}\left\|D_{+}^{t} u^{n}\right\|^{2}+\delta \gamma \Delta x \mu(\Delta x) \Delta t \sum_{n}\left\|D_{+}^{t} D_{-} u^{n}\right\|^{2} \leq \mathcal{F}\left(u_{0}\right)+C T,
\end{aligned}
$$

where

$$
\mathcal{F}\left(u_{0}\right):=\frac{1}{2}\left\|u_{0}\right\|^{2}+\left(\frac{\gamma \mu(\Delta x)}{2}+\frac{\beta \Delta x^{2}}{2}\right)\left\|D_{-} u_{0}\right\|^{2} \leq \frac{1}{2}\left(\left\|u_{0}\right\|^{2}+\left(\frac{\gamma \mu(\Delta x)}{2}+\frac{\beta \Delta x^{2}}{2}\right) \frac{2}{\Delta x^{2}}\left\|u_{0}\right\|^{2}\right) \leq C .
$$

This essentially finishes the proof of the lemma.

Remark 4.4. The assumption B.1 was required to prove the estimate (4.4). This assumption can be relaxed if the primitive $F$ of the given flux function is bounded.

\section{Convergence analysis}

Having obtained all the necessary a priori bounds in the previous section, we are ready to prove that the approximate solutions generated by the scheme (3.2) converge strongly to a weak solution of (1.4), at least along a subsequence. The general strategy of the convergence proof is in the spirit of the one used by DiPerna [5], and it has been used in various contexts by several different authors. However, as we pointed out earlier, we shall use a simplified compensated compactness result in the spirit of ([13], Lem. 3.2). In what follows, using a priori estimates derived in Section 4, we first demonstrate the desired $H_{\text {loc }}^{-1}$ compactness of $\left\{u_{\Delta x}\right\}_{\Delta x>0}$. Then, an application of the compensated compactness Theorem 2.3 gives the desired strong convergence in $L_{\text {loc }}^{p}$ for any $p<\infty$. To achieve our goal, we start with the following crucial lemma:

Lemma 5.1 ( $H_{\mathrm{loc}}^{-1}$ compactness). The sequence

$$
\left\{\eta_{i}\left(k(x), u_{\Delta x}\right)_{t}+q_{i}\left(k(x), u_{\Delta x}\right)_{x}\right\}_{\Delta x>0} \text { is compact in } H_{\text {loc }}^{-1}(\mathbb{R} \times[0, T]),
$$

where $\eta_{i}$ and $q_{i}$ are given by $(2.1)$, for $i=1,2$.

Proof. To begin with, let us assume that $\eta=\eta_{i}$, for $i=1$ or $i=2$, and $\varphi$ be a test function with compact support such that $\varphi(x)=0$, for all $|x|>\left|x_{J+\frac{1}{2}}\right|$, and for $t \geq N \Delta t$, for some $J$ and $N$. With this $\varphi$, we define the following functional

$$
\begin{aligned}
\left\langle\mathcal{L}_{\Delta x}(\eta, q), \varphi\right\rangle & :=-\left\langle\eta\left(k(x), u_{\Delta x}\right)_{t}+q\left(k(x), u_{\Delta x}\right)_{x}, \varphi\right\rangle \\
& =\int_{0}^{T} \int_{\mathbb{R}} \eta\left(k(x), u_{\Delta x}\right) \varphi_{t}+q\left(k(x), u_{\Delta x}\right) \varphi_{x} \mathrm{~d} x \mathrm{~d} t:=\left\langle\mathcal{E}_{\Delta x}(\eta, q), \varphi\right\rangle+\left\langle\mathcal{E}_{\Delta x}^{\prime}(\eta, q), \varphi\right\rangle,
\end{aligned}
$$

where

$$
\left\langle\mathcal{E}_{\Delta x}^{\prime}(\eta, q), \varphi\right\rangle=\int_{0}^{T} \int_{\mathbb{R}}\left(\eta\left(k(x), u_{\Delta x}\right)-\eta\left(k_{\Delta x}, u_{\Delta x}\right)\right) \varphi_{t} \mathrm{~d} x \mathrm{~d} t+\int_{0}^{T} \int_{\mathbb{R}}\left(q\left(k(x), u_{\Delta x}\right)-q\left(k_{\Delta x}, u_{\Delta x}\right)\right) \varphi_{x} \mathrm{~d} x \mathrm{~d} t
$$

and

$$
\left\langle\mathcal{E}_{\Delta x}(\eta, q), \varphi\right\rangle=\int_{0}^{T} \int_{\mathbb{R}} \eta\left(k_{\Delta x}, u_{\Delta x}\right) \varphi_{t}+q\left(k_{\Delta x}, u_{\Delta x}\right) \varphi_{x} \mathrm{~d} x \mathrm{~d} t
$$


In what follows, we let $\Pi_{T}$ denote an arbitrary but fixed bounded open subset of $\mathbb{R} \times[0, T]$. Let $r \in(1,2]$ and set $p=\frac{r}{r-1} \in[1, \infty)$. With $\varphi \in W_{0}^{1, r}\left(\Pi_{T}\right)$, we have by Hölder's inequality

$$
\left|\left\langle\mathcal{E}_{\Delta x}^{\prime}(\eta, q), \varphi\right\rangle\right| \leq C\left\|k-k_{\Delta x}\right\|_{L^{p}\left(\Pi_{T}\right)}\|\varphi\|_{W_{0}^{1, r}\left(\Pi_{T}\right)} \rightarrow 0 \quad \text { as } \Delta x \downarrow 0,
$$

so that

$$
\left\{\mathcal{E}_{\Delta x}^{\prime}(\eta, q)\right\}_{\Delta x>0} \text { is compact in } W^{-1, r}\left(\Pi_{T}\right), \quad r \in(1,2] .
$$

Next we focus on the other term

$$
\begin{aligned}
\left\langle\mathcal{E}_{\Delta x}(\eta, q), \varphi\right\rangle= & \sum_{j} \sum_{n=0}^{N-1} \int_{t^{n}}^{t^{n+1}} \int_{x_{j-\frac{1}{2}}}^{x_{j+\frac{1}{2}}} \eta\left(k^{\Delta x}, u_{j}^{n}\right) \varphi_{t}+q\left(k^{\Delta x}, u_{j}^{n}\right) \varphi_{x} \mathrm{~d} x \mathrm{~d} t \\
= & \sum_{j} \sum_{n=0}^{N-1} \int_{t^{n}}^{t^{n+1}} \int_{x_{j-\frac{1}{2}}}^{x_{j}} \eta\left(k_{j-\frac{1}{2}}, u_{j}^{n}\right) \varphi_{t}+q\left(k_{j-\frac{1}{2}}, u_{j}^{n}\right) \varphi_{x} \mathrm{~d} x \mathrm{~d} t \\
& +\sum_{j} \sum_{n=0}^{N-1} \int_{t^{n}}^{t^{n+1}} \int_{x_{j}}^{x_{j+\frac{1}{2}}} \eta\left(k_{j+\frac{1}{2}}, u_{j}^{n}\right) \varphi_{t}+q\left(k_{j+\frac{1}{2}}, u_{j}^{n}\right) \varphi_{x} \mathrm{~d} x \mathrm{~d} t \\
:= & \left\langle\mathcal{E}_{\Delta x}^{1}(\eta, q), \varphi\right\rangle+\left\langle\mathcal{E}_{\Delta x}^{2}(\eta, q), \varphi\right\rangle
\end{aligned}
$$

where

$$
\left\langle\mathcal{E}_{\Delta x}^{1}(\eta, q), \varphi\right\rangle=\sum_{j} \sum_{n=0}^{N-1} \int_{t^{n}}^{t^{n+1}} \int_{x_{j-\frac{1}{2}}}^{x_{j+\frac{1}{2}}} \eta\left(k_{j-\frac{1}{2}}, u_{j}^{n}\right) \varphi_{t}+q\left(k_{j-\frac{1}{2}}, u_{j}^{n}\right) \varphi_{x} \mathrm{~d} x \mathrm{~d} t
$$

and

$$
\begin{aligned}
\left\langle\mathcal{E}_{\Delta x}^{2}(\eta, q), \varphi\right\rangle= & \sum_{j} \sum_{n=0}^{N-1} \int_{t^{n}}^{t^{n+1}} \int_{x_{j}}^{x_{j+\frac{1}{2}}}\left(\eta\left(k_{j+\frac{1}{2}}, u_{j}^{n}\right)-\eta\left(k_{j-\frac{1}{2}}, u_{j}^{n}\right)\right) \varphi_{t} \mathrm{~d} x \mathrm{~d} t \\
& +\sum_{j} \sum_{n=0}^{N-1} \int_{t^{n}}^{t^{n+1}} \int_{x_{j}}^{x_{j+\frac{1}{2}}}\left(q\left(k_{j+\frac{1}{2}}, u_{j}^{n}\right)-q\left(k_{j-\frac{1}{2}}, u_{j}^{n}\right)\right) \varphi_{x} \mathrm{~d} x \mathrm{~d} t .
\end{aligned}
$$

The proof is essentially complete if we assume that both $\left\{\mathcal{E}_{\Delta x}^{1}(\eta, q)\right\}_{\Delta x>0}$ and $\left\{\mathcal{E}_{\Delta x}^{2}(\eta, q)\right\}_{\Delta x>0}$ are compact in $H_{\mathrm{loc}}^{-1}\left(\Pi_{T}\right)$.

In the proof Lemma 5.1, we claimed the compactness of $\left\{\mathcal{E}_{\Delta x}^{1}(\eta, q)\right\}_{\Delta x>0}$ and $\left\{\mathcal{E}_{\Delta x}^{2}(\eta, q)\right\}_{\Delta x>0}$. We try to justify this claim below. For the rest of this section, we assume that the conditions stated in Lemma 5.1 hold. We will also continue to use $\Pi_{T}$ introduced in the proof of the above lemma.

\section{Lemma 5.2.}

$$
\left\{\mathcal{E}_{\Delta x}^{2}(\eta, q)\right\}_{\Delta x>0} \text { is compact in } H_{\mathrm{loc}}^{-1}\left(\Pi_{T}\right) .
$$

Proof. Consider the expression given by (5.3), which is split as

$$
\left\langle\mathcal{E}_{\Delta x}^{2}(\eta, q), \varphi\right\rangle:=\left\langle\mathcal{E}_{\Delta x}^{2,1}(\eta, q), \varphi\right\rangle+\left\langle\mathcal{E}_{\Delta x}^{2,2}(\eta, q), \varphi\right\rangle
$$


where

$$
\begin{aligned}
& \left\langle\mathcal{E}_{\Delta x}^{2,1}(\eta, q), \varphi\right\rangle=\sum_{j} \sum_{n=0}^{N-1} \int_{t^{n}}^{t^{n+1}} \int_{x_{j}}^{x_{j+\frac{1}{2}}}\left(\eta\left(k_{j+\frac{1}{2}}, u_{j}^{n}\right)-\eta\left(k_{j-\frac{1}{2}}, u_{j}^{n}\right)\right) \varphi_{t} \mathrm{~d} x \mathrm{~d} t \\
& \left\langle\mathcal{E}_{\Delta x}^{2,2}(\eta, q), \varphi\right\rangle=\sum_{j} \sum_{n=0}^{N-1} \int_{t^{n}}^{t^{n+1}} \int_{x_{j}}^{x_{j+\frac{1}{2}}}\left(q\left(k_{j+\frac{1}{2}}, u_{j}^{n}\right)-q\left(k_{j-\frac{1}{2}}, u_{j}^{n}\right)\right) \varphi_{x} \mathrm{~d} x \mathrm{~d} t .
\end{aligned}
$$

Since $\eta^{\prime}$ is bounded, a simple application of Cauchy-Schwartz inequality implies

$$
\begin{aligned}
\left|\left\langle\mathcal{E}_{\Delta x}^{2,1}(\eta, q), \varphi\right\rangle\right| & \leq \sum_{j} \sum_{n=0}^{N-1} \int_{t^{n}}^{t^{n+1}} \int_{x_{j}}^{x_{j+\frac{1}{2}}}\left|\eta\left(k_{j+\frac{1}{2}}, u_{j}^{n}\right)-\eta\left(k_{j-\frac{1}{2}}, u_{j}^{n}\right)\right|\left|\varphi_{t}\right| \mathrm{d} x \mathrm{~d} t \\
& \leq C \sum_{j} \int_{0}^{T} \int_{x_{j}}^{x_{j+\frac{1}{2}}}\left|k_{j+\frac{1}{2}}-k_{j-\frac{1}{2}}\right|\left|\varphi_{t}\right| \mathrm{d} x \mathrm{~d} t \\
& \leq C \sum_{j} \int_{0}^{T}\left|k_{j+\frac{1}{2}}-k_{j-\frac{1}{2}}\right|(\Delta x)^{\frac{1}{2}}\left(\int_{x_{j}}^{x_{j+\frac{1}{2}}}\left|\varphi_{t}\right|^{2} \mathrm{~d} x\right)^{\frac{1}{2}} \mathrm{~d} t
\end{aligned}
$$

Applying Cauchy-Schwartz a few more times, we get the estimate

$$
\begin{aligned}
\left|\left\langle\mathcal{E}_{\Delta x}^{2,1}(\eta, q), \varphi\right\rangle\right| & \leq C(\Delta x)^{\frac{1}{2}} \int_{0}^{T}\left(\sum_{j}\left|k_{j+\frac{1}{2}}-k_{j-\frac{1}{2}}\right|^{2}\right)^{\frac{1}{2}}\left(\sum_{j} \int_{x_{j}}^{x_{j+\frac{1}{2}}}\left|\varphi_{t}\right|^{2} \mathrm{~d} x\right)^{\frac{1}{2}} \mathrm{~d} t \\
& \leq C(\Delta x)^{\frac{1}{2}}\left(\int_{0}^{T} \sum_{j}\left|k_{j+\frac{1}{2}}-k_{j-\frac{1}{2}}\right|^{2} \mathrm{~d} t\right)^{\frac{1}{2}}\left(\int_{0}^{T} \sum_{j} \int_{x_{j}}^{x_{j+\frac{1}{2}}}\left|\varphi_{t}\right|^{2} \mathrm{~d} x \mathrm{~d} t\right)^{\frac{1}{2}} \\
& \leq C(\Delta x)^{\frac{1}{2}}|k|_{B V} T^{1 / 2}\|\varphi\|_{H^{1}\left(\Pi_{T}\right)} .
\end{aligned}
$$

A similar argument can be used to show almost verbatim

$$
\left|\left\langle\mathcal{E}_{\Delta x}^{2,2}(\eta, q), \varphi\right\rangle\right| \leq C(\Delta x)^{\frac{1}{2}}|k|_{B V} T^{1 / 2}\|\varphi\|_{H^{1}\left(\Pi_{T}\right)} .
$$

Therefore, summing up, we have

$$
\left|\left\langle\mathcal{E}_{\Delta x}^{2}(\eta, q), \varphi\right\rangle\right| \leq C(\Delta x)^{\frac{1}{2}}|k|_{B V} T^{1 / 2}\|\varphi\|_{H^{1}\left(\Pi_{T}\right)} .
$$

Thus,

$$
\left\{\mathcal{E}_{\Delta x}^{2}(\eta, q)\right\}_{\Delta x>0} \text { is compact in } H_{\mathrm{loc}}^{-1}\left(\Pi_{T}\right) .
$$

Next, we need to show the compactness of $\left\{\mathcal{E}_{\Delta x}^{1}(\eta, q)\right\}_{\Delta x>0}$. First note that we can express (5.2) as

$$
\begin{aligned}
\left\langle\mathcal{E}_{\Delta x}^{1}(\eta, q), \varphi\right\rangle= & -\Delta t \sum_{j} \sum_{n=0}^{N-1} \int_{x_{j-\frac{1}{2}}}^{x_{j+\frac{1}{2}}} D_{+}^{t} \eta\left(k_{j-\frac{1}{2}}, u_{j}^{n}\right) \varphi^{n+1}(x) \mathrm{d} x \\
& -\sum_{j} \int_{I_{j}} \eta\left(k_{j-\frac{1}{2}}, u_{j}^{0}\right) \varphi^{0}(x) \mathrm{d} x-\Delta x \sum_{j} \sum_{n=0}^{N-1} \int_{t^{n}}^{t^{n+1}} D_{-} q\left(k_{j-\frac{1}{2}}, u_{j}^{n}\right) \varphi_{j-\frac{1}{2}}(t) \mathrm{d} t,
\end{aligned}
$$


where $\varphi_{j-\frac{1}{2}}(t):=\varphi\left(x_{j-\frac{1}{2}}, t\right)$, and $\varphi^{n+1}(x):=\varphi\left(x, t^{n+1}\right)$. This further implies that

$$
\begin{aligned}
-\left\langle\mathcal{E}_{\Delta x}^{1}(\eta, q), \varphi\right\rangle= & \sum_{j} \sum_{n=0}^{N-1} \int_{t^{n}}^{t^{n+1}} \int_{x_{j-\frac{1}{2}}}^{x_{j+\frac{1}{2}}}\left(D_{+}^{t} \eta\left(k_{j-\frac{1}{2}}, u_{j}^{n}\right)+D_{-} q\left(k_{j-\frac{1}{2}}, u_{j}^{n}\right)\right) \varphi \mathrm{d} x \mathrm{~d} t \\
& +\sum_{j} \sum_{n=0}^{N-1} \int_{t^{n}}^{t^{n+1}} \int_{x_{j-\frac{1}{2}}}^{x_{j+\frac{1}{2}}} D_{-} q\left(k_{j-\frac{1}{2}}, u_{j}^{n}\right)\left(\varphi_{j-\frac{1}{2}}(t)-\varphi(x, t)\right) \mathrm{d} x \mathrm{~d} t \\
& +\sum_{j} \sum_{n=0}^{N-1} \int_{t^{n}}^{t^{n+1}} \int_{x_{j-\frac{1}{2}}}^{x_{j+\frac{1}{2}}} D_{+}^{t} \eta\left(k_{j-\frac{1}{2}}, u_{j}^{n}\right)\left(\varphi^{n+1}(x)-\varphi(x, t)\right) \mathrm{d} x \mathrm{~d} t \\
& +\sum_{j} \int_{I_{j}} \eta\left(k_{j-\frac{1}{2}}, u_{j}^{0}\right) \varphi^{0}(x) \mathrm{d} x \\
:= & \left\langle\mathcal{E}_{\Delta x}^{1,1}(\eta, q), \varphi\right\rangle+\left\langle\mathcal{E}_{\Delta x}^{1,2}(\eta, q), \varphi\right\rangle
\end{aligned}
$$

where

$$
\left\langle\mathcal{E}_{\Delta x}^{1,1}(\eta, q), \varphi\right\rangle=\sum_{j} \sum_{n=0}^{N-1} \int_{t^{n}}^{t^{n+1}} \int_{x_{j-\frac{1}{2}}}^{x_{j+\frac{1}{2}}}\left(D_{+}^{t} \eta\left(k_{j-\frac{1}{2}}, u_{j}^{n}\right)+D_{-} q\left(k_{j-\frac{1}{2}}, u_{j}^{n}\right)\right) \varphi \mathrm{d} x \mathrm{~d} t
$$

while $\left\langle\mathcal{E}_{\Delta x}^{1,2}(\eta, q), \varphi\right\rangle$ corresponds to the three remaining summation term. The compactness of $\left.\left\{\mathcal{E}_{\Delta x}^{1,1}(\eta, q)\right\}\right\}_{\Delta x>0}$ and $\left\{\mathcal{E}_{\Delta x}^{1,2}(\eta, q)\right\}_{\Delta x>0}$ will ensure the compactness of $\left\{\mathcal{E}_{\Delta x}^{1}(\eta, q)\right\}_{\Delta x>0}$

\section{Lemma 5.3.}

$$
\left\{\mathcal{E}_{\Delta x}^{1,2}(\eta, q)\right\}_{\Delta x>0} \text { is compact in } H_{\mathrm{loc}}^{-1}\left(\Pi_{T}\right)
$$

Proof. Firstly, we split the $\mathcal{E}_{\Delta x}^{1,2}(\eta, q)$ as

$$
\left.\left\langle\mathcal{E}_{\Delta x}^{1,2}(\eta, q), \varphi\right\rangle:=\left\langle\mathcal{E}_{\Delta x}^{1,2,1}(\eta, q), \varphi\right\rangle+\left\langle\mathcal{E}_{\Delta x}^{1,2,2}(\eta, q), \varphi\right\rangle+\mathcal{E}_{\Delta x}^{1,2,3}(\eta, q), \varphi\right\rangle
$$

where

$$
\begin{aligned}
& \left\langle\mathcal{E}_{\Delta x}^{1,2,1}(\eta, q), \varphi\right\rangle=\sum_{j} \sum_{n=0}^{N-1} \int_{t^{n}}^{t^{n+1}} \int_{x_{j-\frac{1}{2}}}^{x_{j+\frac{1}{2}}} D_{-} q\left(k_{j-\frac{1}{2}}, u_{j}^{n}\right)\left(\varphi_{j-\frac{1}{2}}(t)-\varphi(x, t)\right) \mathrm{d} x \mathrm{~d} t \\
& \left\langle\mathcal{E}_{\Delta x}^{1,2,2}(\eta, q), \varphi\right\rangle=\sum_{j} \sum_{n=0}^{N-1} \int_{t^{n}}^{t^{n+1}} \int_{x_{j-\frac{1}{2}}}^{x_{j+\frac{1}{2}}} D_{+}^{t} \eta\left(k_{j-\frac{1}{2}}, u_{j}^{n}\right)\left(\varphi^{n+1}(x)-\varphi(x, t)\right) \mathrm{d} x \mathrm{~d} t \\
& \left\langle\mathcal{E}_{\Delta x}^{1,2,3}(\eta, q), \varphi\right\rangle=\sum_{j} \int_{I_{j}} \eta\left(k_{j-\frac{1}{2}}, u_{j}^{0}\right) \varphi^{0}(x) \mathrm{d} x .
\end{aligned}
$$


We first estimate the term $\mathcal{E}_{\Delta x}^{1,2,1}(\eta, q)$ as follows:

$$
\begin{aligned}
\left\langle\mathcal{E}_{\Delta x}^{1,2,1}(\eta, q), \varphi\right\rangle= & \sum_{j, n} \int_{t^{n}}^{t^{n+1}} \int_{I_{j}} \frac{q\left(k_{j-\frac{1}{2}}, u_{j}^{n}\right)-q\left(k_{j-\frac{3}{2}}, u_{j-1}^{n}\right)}{\Delta x}\left(\varphi_{j-\frac{1}{2}}-\varphi(x, t)\right) \mathrm{d} x \mathrm{~d} t \\
= & \underbrace{\sum_{j} \sum_{n=0}^{N-1} \int_{t^{n}}^{t^{n+1}} \int_{x_{j-\frac{1}{2}}}^{x_{j+\frac{1}{2}}} \frac{q\left(k_{j-\frac{1}{2}}, u_{j}^{n}\right)-q\left(k_{j-\frac{1}{2}}, u_{j-1}^{n}\right)}{\Delta x}\left(\varphi_{j-\frac{1}{2}}-\varphi(x, t)\right) \mathrm{d} x \mathrm{~d} t}_{\left\langle I_{\Delta x}(\eta, q), \varphi\right\rangle} \\
& +\underbrace{\sum_{j} \sum_{n=0}^{N-1} \int_{t^{n}}^{t^{n+1}} \int_{x_{j-\frac{1}{2}}}^{x_{j+\frac{1}{2}}} \frac{q\left(k_{j-\frac{1}{2}}, u_{j-1}^{n}\right)-q\left(k_{j-\frac{3}{2}}, u_{j-1}^{n}\right)}{\Delta x}\left(\varphi_{j-\frac{1}{2}}-\varphi(x, t)\right) \mathrm{d} x \mathrm{~d} t}_{\left\langle I_{\Delta x}(\eta), \varphi\right\rangle} .
\end{aligned}
$$

Since $q^{\prime}$ and $\varphi$ are both bounded, and $k$ is of bounded variation, we see that

$$
\left|\left\langle I I_{\Delta x}(\eta, q), \varphi\right\rangle\right| \leq C(T)\|\varphi\|_{L^{\infty}\left(\Pi_{T}\right)}|k|_{B V} .
$$

Hence we conclude that

$$
I I_{\Delta x}(\eta, q) \in \mathcal{M}_{\mathrm{loc}}\left(\Pi_{T}\right) .
$$

Now the term $I_{\Delta x}(\eta, q)$ can be estimated, using Cauchy-Schwartz inequality repeatedly, as follows

$$
\begin{gathered}
\left|\left\langle I_{\Delta x}(\eta, q), \varphi\right\rangle\right| \leq C \sum_{j} \sum_{n=0}^{N-1} \int_{t^{n}}^{t^{n+1}} \int_{x_{j-\frac{1}{2}}}^{x_{j+\frac{1}{2}}}\left|\frac{u_{j}^{n}-u_{j-1}^{n}}{\Delta x}\right| \int_{x_{j-\frac{1}{2}}}^{x}\left|\varphi_{x}(\theta)\right| d \theta \mathrm{d} x \mathrm{~d} t \\
\leq(\Delta x)^{\frac{1}{2}} C \sum_{j} \sum_{n=0}^{N-1} \int_{t^{n}}^{t^{n+1}} \int_{x_{j-\frac{1}{2}}}^{x_{j+\frac{1}{2}}}\left|\frac{u_{j}^{n}-u_{j-1}^{n}}{\Delta x}\right|\left(\int_{x_{j-\frac{1}{2}}}^{x}\left|\varphi_{x}(\theta)\right|^{2} d \theta\right)^{1 / 2} \mathrm{~d} x \mathrm{~d} t \\
\leq(\Delta x)^{\frac{1}{2}} C \sum_{j} \sum_{n=0}^{N-1} \int_{t^{n}}^{t^{n+1}}\left|u_{j}^{n}-u_{j-1}^{n}\right|\left(\int_{x_{j-\frac{1}{2}}}^{x_{j+\frac{1}{2}}}\left|\varphi_{x}\right|^{2} \mathrm{~d} x\right)^{1 / 2} \mathrm{~d} t \\
\leq(\Delta x)^{\frac{1}{2}} C\left(\Delta t \sum_{n=0}^{N-1} \sum_{j} \int_{t^{n}}^{t^{n+1}}\left|u_{j}^{n}-u_{j-1}^{n}\right|^{2} \mathrm{~d} t\right)^{\frac{1}{2}}\|\varphi\|_{H^{1}\left(\Pi_{T}\right)} .
\end{gathered}
$$

Therefore, in view of the a priori bound $(4.3 \mathrm{~b})$, we reach to the conclusion that

$$
\left\{I_{\Delta x}(\eta, q)\right\}_{\Delta x>0} \text { is compact in } H_{\mathrm{loc}}^{-1}\left(\Pi_{T}\right) .
$$

To sum up, making use of (5.7) and (5.8), along with Lemma 2.4, we conclude that

$$
\left\{\mathcal{E}_{\Delta x}^{1,2,1}(\eta, q)\right\}_{\Delta x>0} \text { is compact in } H_{\text {loc }}^{-1}\left(\Pi_{T}\right) .
$$

A similar type of argument, with the use of Cauchy-Schwartz inequality, yields

$$
\left|\left\langle\mathcal{E}_{\Delta x}^{1,2,2}(\eta, q), \varphi\right\rangle\right| \leq C(\Delta t)^{1 / 2}\left(\Delta t \Delta x^{2} \sum_{n=0}^{N} \sum_{j}\left(D_{+}^{t} u_{j}^{n}\right)^{2}\right)^{1 / 2}\|\varphi\|_{H^{1}\left(\Pi_{T}\right)} .
$$


Making use of the a priori bound (4.3c), we conclude that

$$
\left\{\mathcal{E}_{\Delta x}^{1,2,2}(\eta, q)\right\}_{\Delta x>0} \text { is compact in } H_{\text {loc }}^{-1}\left(\Pi_{T}\right) .
$$

Moreover, note that the bound

$$
\left|\left\langle\mathcal{E}_{\Delta x}^{1,2,3}(\eta, q), \varphi\right\rangle\right| \leq C\|\varphi\|_{L^{\infty}(\mathbb{R})}
$$

ensures that

$$
\left\{\mathcal{E}_{\Delta x}^{1,2,3}(\eta, q)\right\}_{\Delta x>0} \in \mathcal{M}_{\mathrm{loc}}\left(\Pi_{T}\right)
$$

Using (5.9),(5.10) and (5.11) along the lines of Lemma 2.4, proves that $\left\{\mathcal{E}_{\Delta x}^{1,2}(\eta, q)\right\}_{\Delta x>0}$ is compact.

Next we estimate the remaining term $\mathcal{E}_{\Delta x}^{1,1}(\eta, q)$. We first note that Taylor's series expansion yields

$$
\eta_{u}\left(k_{j-\frac{1}{2}}, u_{j}^{n}\right) D_{+}^{t} u_{j}^{n}=D_{+}^{t} \eta\left(k_{j-\frac{1}{2}}, u_{j}^{n}\right)-\frac{\Delta t}{2} \eta_{u u}\left(k_{j-\frac{1}{2}}, \theta_{j}^{n+\frac{1}{2}}\right)\left(D_{+}^{t} u_{j}^{n}\right)^{2},
$$

for some $\theta_{j}^{n+\frac{1}{2}}$ between $u_{j}^{n}$ and $u_{j}^{n+1}$. At this point, we shall make use of the fully-discrete scheme (3.2) and (5.12) to decompose the term $\mathcal{E}_{\Delta x}^{1,1}(\eta, q)$ as follows:

$$
\begin{aligned}
\left\langle\mathcal{E}_{\Delta x}^{1,1}(\eta, q), \varphi\right\rangle= & \sum_{j} \sum_{n=0}^{N-1} \int_{t^{n}}^{t^{n+1}} \int_{x_{j-\frac{1}{2}}}^{x_{j+\frac{1}{2}}}\left(D_{+}^{t} \eta\left(k_{j-\frac{1}{2}}, u_{j}^{n}\right)+D_{-} q\left(k_{j-\frac{1}{2}}, u_{j}^{n}\right)\right) \varphi \mathrm{d} x \mathrm{~d} t \\
& :=\left\langle\mathcal{E}_{\Delta x}^{1,1,1}(\eta, q), \varphi\right\rangle+\left\langle\mathcal{E}_{\Delta x}^{1,1,2}(\eta, q), \varphi\right\rangle+\left\langle\mathcal{E}_{\Delta x}^{1,1,3}(\eta, q), \varphi\right\rangle+\left\langle\mathcal{E}_{\Delta x}^{1,1,4}(\eta, q), \varphi\right\rangle,
\end{aligned}
$$

where

$$
\begin{aligned}
\left\langle\mathcal{E}_{\Delta x}^{1,1,1}(\eta, q), \varphi\right\rangle & =\sum_{j} \sum_{n=0}^{N-1} \int_{t^{n}}^{t^{n+1}} \int_{I_{j}}\left[-\eta_{u}\left(k_{j-\frac{1}{2}}, u_{j}^{n}\right) D_{-}\left(k_{j+\frac{1}{2}} \hat{f}_{j+\frac{1}{2}}^{n}\right)+D_{-} q\left(k_{j-\frac{1}{2}}, u_{j}^{n}\right)\right] \varphi \mathrm{d} x \mathrm{~d} t \\
\left\langle\mathcal{E}_{\Delta x}^{1,1,2}(\eta, q), \varphi\right\rangle & =\beta \sum_{j} \sum_{n=0}^{N-1} \int_{t^{n}}^{t^{n+1}} \int_{I_{j}} \eta_{u}\left(k_{j-\frac{1}{2}}, u_{j}^{n}\right) \Delta x\left(D_{+} D_{-} u_{j}^{n}\right) \varphi \mathrm{d} x \mathrm{~d} t \\
\left\langle\mathcal{E}_{\Delta x}^{1,1,3}(\eta, q), \varphi\right\rangle & =\gamma \sum_{j} \sum_{n=0}^{N-1} \int_{t^{n}}^{t^{n+1}} \int_{I_{j}} \eta_{u}\left(k_{j-\frac{1}{2}}, u_{j}^{n}\right) \mu(\Delta x)\left(D_{+}^{t} D_{+} D_{-} u_{j}^{n}\right) \varphi \mathrm{d} x \mathrm{~d} t \\
\left\langle\mathcal{E}_{\Delta x}^{1,1,4}(\eta, q), \varphi\right\rangle & =\sum_{j} \sum_{n=0}^{N-1} \int_{t^{n}}^{t^{n+1}} \int_{I_{j}} \frac{\Delta t}{2} \eta_{u u}\left(k_{j-\frac{1}{2}}, \theta_{j}^{n+\frac{1}{2}}\right)\left(D_{+}^{t} u_{j}^{n}\right)^{2} \varphi \mathrm{d} x \mathrm{~d} t .
\end{aligned}
$$

Our aim is to estimate each of the above terms suitably. In order to do so, we introduce the notation $\Phi_{j}:=$ $\int_{x_{j-\frac{1}{2}}}^{x_{j+\frac{1}{2}}} \varphi(x, t) \mathrm{d} x$.

\section{Lemma 5.4.}

$$
\left\{\mathcal{E}_{\Delta x}^{1,1,1}(\eta, q)\right\}_{\Delta x>0} \text { is compact in } H_{\mathrm{loc}}^{-1}\left(\Pi_{T}\right) \text {. }
$$


Proof. We rewrite $\mathcal{E}_{\Delta x}^{1,1,1}(\eta, q)$ as

$$
\begin{aligned}
\left\langle\mathcal{E}_{\Delta x}^{1,1,1}(\eta, q), \varphi\right\rangle= & \sum_{j} \sum_{n=0}^{N-1} \int_{t^{n}}^{t^{n+1}}\left[-\eta_{u}\left(k_{j-\frac{1}{2}}, u_{j}^{n}\right) D_{-}\left(k_{j+\frac{1}{2}} \hat{f}_{j+\frac{1}{2}}^{n}\right)+D_{-} q\left(k_{j-\frac{1}{2}}, u_{j}^{n}\right)\right] \Phi_{j} \mathrm{~d} t \\
= & \sum_{n=0}^{N-1} \int_{t^{n}}^{t^{n+1}} \sum_{j}\left[-\eta_{u}\left(k_{j-\frac{1}{2}}, u_{j}^{n}\right)\left(k_{j-\frac{1}{2}} D_{-} \hat{f}_{j+\frac{1}{2}}^{n}+\hat{f}_{j+\frac{1}{2}}^{n} D_{-} k_{j+\frac{1}{2}}\right)\right. \\
& \left.+\frac{q\left(k_{j-\frac{1}{2}}, u_{j}^{n}\right)-q\left(k_{j-\frac{3}{2}}, u_{j-1}^{n}\right)}{\Delta x}\right] \Phi_{j} \mathrm{~d} t \\
= & \sum_{n=0}^{N-1} \int_{t^{n}}^{t^{n+1}} \sum_{j}\left[-\eta_{u}\left(k_{j-\frac{1}{2}}, u_{j}^{n}\right) k_{j-\frac{1}{2}} D_{-} \hat{f}_{j+\frac{1}{2}}^{n}\right. \\
& +\sum_{n=0}^{N-1} \int_{t^{n}}^{t^{n+1}} \sum_{j}\left[-\eta_{u}\left(k_{j-\frac{1}{2}}, u_{j}^{n}\right) \hat{f}_{j+\frac{1}{2}}^{n} D_{-} k_{j+\frac{1}{2}}\right. \\
& \left.+\frac{q\left(k_{j-\frac{1}{2}}, u_{j}^{n}\right)-q\left(k_{j-\frac{1}{2}}, u_{j-1}^{n}\right)}{\Delta x}\right] \Phi_{j} \mathrm{~d} t \\
:= & \left\langle\mathcal{E}_{\Delta x}^{1,1,1,1}(\eta, q), \varphi\right\rangle+\left\langle\mathcal{E}_{\Delta x}^{1,1,1,2}(\eta, q), \varphi\right\rangle
\end{aligned}
$$

where

$$
\begin{aligned}
\left\langle\mathcal{E}_{\Delta x}^{1,1,1,1}(\eta, q), \varphi\right\rangle & :=\sum_{n=0}^{N-1} \int_{t^{n}}^{t^{n+1}} \sum_{j}\left[-\eta_{u}\left(k_{j-\frac{1}{2}}, u_{j}^{n}\right) k_{j-\frac{1}{2}} D_{-} \hat{f}_{j+\frac{1}{2}}^{n}+\frac{q\left(k_{j-\frac{1}{2}}, u_{j}^{n}\right)-q\left(k_{j-\frac{1}{2}}, u_{j-1}^{n}\right)}{\Delta x}\right] \Phi_{j} \mathrm{~d} t \\
\left\langle\mathcal{E}_{\Delta x}^{1,1,1,2}(\eta, q), \varphi\right\rangle & :=\sum_{n=0}^{N-1} \int_{t^{n}}^{t^{n+1}} \sum_{j}\left[-\eta_{u}\left(k_{j-\frac{1}{2}}, u_{j}^{n}\right) \hat{f}_{j+\frac{1}{2}}^{n} D_{-} k_{j+\frac{1}{2}}+\frac{q\left(k_{j-\frac{1}{2}}, u_{j-1}^{n}\right)-q\left(k_{j-\frac{3}{2}}, u_{j-1}^{n}\right)}{\Delta x}\right] \Phi_{j} \mathrm{~d} t .
\end{aligned}
$$

Since $\eta^{\prime}$ and $q^{\prime}$ are bounded, making use the total variation bound for $k$, we conclude that

$$
\left|\left\langle\mathcal{E}_{\Delta x}^{1,1,1,2}(\eta, q), \varphi\right\rangle\right| \leq C|k|_{B V}\|\varphi\|_{L^{\infty}\left(\Pi_{T}\right)} .
$$

Hence this implies

$$
\left\{\mathcal{E}_{\Delta x}^{1,1,1,2}(\eta, q)\right\}_{\Delta x>0} \in \mathcal{M}_{\mathrm{loc}}\left(\Pi_{T}\right)
$$

Next we move on to estimate $\mathcal{E}_{\Delta x}^{1,1,1,1}(\eta, q)$. At this point we shall make use of specific structure of $\eta$ and $q$ given by (2.1).

Estimate for $(\eta, q):=\left(\eta_{1}, q_{1}\right):$ First, we recall that

$$
\eta_{1}(u)=u-c, \quad q_{1}(k, u)=f(k, u)-f(k, c)=k(x)(f(u)-f(c)) .
$$


Thus, we find that

$$
\begin{aligned}
-\frac{\partial}{\partial u} \eta_{1}\left(k_{j-\frac{1}{2}}, u_{j}^{n}\right) & k_{j-\frac{1}{2}} D_{-} \hat{f}_{j+\frac{1}{2}}^{n}+\frac{q_{1}\left(k_{j-\frac{1}{2}}, u_{j}^{n}\right)-q_{1}\left(k_{j-\frac{1}{2}}, u_{j-1}^{n}\right)}{\Delta x} \\
& =-k_{j-\frac{1}{2}} \frac{\hat{f}_{j+\frac{1}{2}}^{n}-\hat{f}_{j-\frac{1}{2}}^{n}}{\Delta x}+k_{j-\frac{1}{2}} \frac{f\left(u_{j}^{n}\right)-f\left(u_{j-1}^{n}\right)}{\Delta x} \\
& =\frac{k_{j-\frac{1}{2}}}{\Delta x}\left[\left(f\left(u_{j}^{n}\right)-\hat{f}_{j+\frac{1}{2}}^{n}\right)-\left(f\left(u_{j-1}^{n}\right)-\hat{f}_{j-\frac{1}{2}}^{n}\right)\right]=k_{j-\frac{1}{2}} D_{-}\left(f\left(u_{j}^{n}\right)-\hat{f}_{j+\frac{1}{2}}^{n}\right) .
\end{aligned}
$$

We insert this in the expression of $\left\langle\mathcal{E}_{\Delta x}^{1,1,1,1}(\eta, q), \varphi\right\rangle$. Then, using summation by parts we obtain

$$
\begin{aligned}
\left\langle\mathcal{E}_{\Delta x}^{1,1,1,1}\left(\eta_{1}, q_{1}\right), \varphi\right\rangle & =\sum_{n=0}^{N-1} \int_{t^{n}}^{t^{n+1}} \sum_{j} k_{j-\frac{1}{2}} D_{-}\left(f\left(u_{j}^{n}\right)-\hat{f}_{j+\frac{1}{2}}^{n}\right) \Phi_{j} \mathrm{~d} t \\
& =-\sum_{n=0}^{N-1} \int_{t^{n}}^{t^{n+1}} \sum_{j}\left(f\left(u_{j}^{n}\right)-\hat{f}_{j+\frac{1}{2}}^{n}\right) D_{+}\left(k_{j-\frac{1}{2}} \Phi_{j}\right) \mathrm{d} t \\
& =-\sum_{n=0}^{N-1} \int_{t^{n}}^{t^{n+1}} \sum_{j}\left(f\left(u_{j}^{n}\right)-\hat{f}_{j+\frac{1}{2}}^{n}\right)\left[k_{j+\frac{1}{2}} D_{+} \Phi_{j}+\Phi_{j} D_{+} k_{j-\frac{1}{2}}\right] \mathrm{d} t \\
& :=\left\langle I_{\Delta x}\left(\eta_{1}, q_{1}\right), \varphi\right\rangle+\left\langle I I_{\Delta x}\left(\eta_{1}, q_{1}\right), \varphi\right\rangle .
\end{aligned}
$$

To proceed further, we first estimate the term $I_{\Delta x}\left(\eta_{1}, q_{1}\right)$ as follows

$$
\begin{aligned}
\left|\left\langle I_{\Delta x}\left(\eta_{1}, q_{1}\right), \varphi\right\rangle\right| & =\left|-\sum_{n=0}^{N-1} \int_{t^{n}}^{t^{n+1}} \sum_{j}\left(f\left(u_{j}^{n}\right)-\hat{f}_{j+\frac{1}{2}}^{n}\right) k_{j+\frac{1}{2}} D_{+} \Phi_{j} \mathrm{~d} t\right| \\
& \leq\|k\|_{\infty}\left\|f^{\prime}\right\|_{\infty} \sum_{n=0}^{N-1} \int_{t^{n}}^{t^{n+1}} \sum_{j}\left|u_{j}^{n}-u_{j+1}^{n}\right|\left|D_{+} \Phi_{j}\right| \mathrm{d} t \\
& =\|k\|_{\infty}\left\|f^{\prime}\right\|_{\infty} \sum_{n=0}^{N-1} \int_{t^{n}}^{t^{n+1}} \sum_{j}\left|u_{j}^{n}-u_{j-1}^{n}\right|\left|D_{-} \Phi_{j}\right| \mathrm{d} t .
\end{aligned}
$$

Next, we estimate $\left|D_{-} \Phi_{j}\right|$ as follows

$$
\begin{aligned}
\Phi_{j}-\Phi_{j-1} & =\int_{x_{j-\frac{1}{2}}}^{x_{j+\frac{1}{2}}} \varphi \mathrm{d} x-\int_{x_{j-\frac{3}{2}}}^{x_{j-\frac{1}{2}}} \varphi \mathrm{d} x=\int_{x_{j-\frac{1}{2}}}^{x_{j+\frac{1}{2}}}\left(\varphi-\varphi_{j-\frac{1}{2}}\right) \mathrm{d} x-\int_{x_{j-\frac{3}{2}}}^{x_{j-\frac{1}{2}}}\left(\varphi-\varphi_{j-\frac{1}{2}}\right) \mathrm{d} x \\
& =\int_{x_{j-\frac{1}{2}}}^{x_{j+\frac{1}{2}}} \int_{x_{j-\frac{1}{2}}}^{x} \varphi_{x} \mathrm{~d} \theta \mathrm{d} x-\int_{x_{j-\frac{3}{2}}}^{x_{j-\frac{1}{2}}} \int_{x_{j-\frac{1}{2}}}^{x} \varphi_{x} \mathrm{~d} \theta \mathrm{d} x .
\end{aligned}
$$

Therefore,

$$
\begin{aligned}
\left|\Phi_{j}-\Phi_{j-1}\right| & \leq \int_{x_{j-\frac{1}{2}}}^{x_{j+\frac{1}{2}}} \int_{x_{j-\frac{1}{2}}}^{x}\left|\varphi_{x}\right| \mathrm{d} \theta \mathrm{d} x+\int_{x_{j-\frac{3}{2}}}^{x_{j-\frac{1}{2}}} \int_{x_{j-\frac{1}{2}}}^{x}\left|\varphi_{x}\right| \mathrm{d} \theta \mathrm{d} x \\
& \leq 2 \Delta x \int_{x_{j-\frac{1}{2}}}^{x_{j+\frac{1}{2}}}\left|\varphi_{x}\right| \mathrm{d} x \leq 2(\Delta x)^{\frac{3}{2}}\left(\int_{x_{j-\frac{1}{2}}}^{x_{j+\frac{1}{2}}}\left|\varphi_{x}\right|^{2} \mathrm{~d} x\right)^{1 / 2}
\end{aligned}
$$


Thus, we conclude that

$$
\left|D \_\Phi_{j}\right| \leq 2(\Delta x)^{1 / 2}\left(\int_{x_{j-\frac{1}{2}}}^{x_{j+\frac{1}{2}}}\left|\varphi_{x}\right|^{2} \mathrm{~d} x\right)^{1 / 2} .
$$

Therefore, applying Cauchy-Schwartz inequality and using the a priori estimate (4.3b), we obtain

$$
\begin{aligned}
\left|\left\langle I_{\Delta x}\left(\eta_{1}, q_{1}\right), \varphi\right\rangle\right| & \leq 2\|k\|_{\infty}\left\|f^{\prime}\right\|_{\infty}(\Delta x)^{1 / 2}\left(\sum_{n=0}^{N} \Delta t \Delta x\left\|D_{-} u^{n}\right\|^{2}\right)^{1 / 2}\|\varphi\|_{H^{1}\left(\Pi_{T}\right)} \\
& \leq C(\Delta x)^{1 / 2}\|\varphi\|_{H^{1}\left(\Pi_{T}\right)} .
\end{aligned}
$$

Hence

$$
\left\{I_{\Delta x}\left(\eta_{1}, q_{1}\right)\right\}_{\Delta x>0} \text { is compact in } H_{\mathrm{loc}}^{-1}\left(\Pi_{T}\right) .
$$

On the other hand, making use of the total variation bound for $k$, we have

$$
\left|\left\langle I I_{\Delta x}\left(\eta_{1}, q_{1}\right), \varphi\right\rangle\right| \leq C\|\varphi\|_{L^{\infty}\left(\Pi_{T}\right)}|k|_{B V} .
$$

Therefore

$$
\left\{I I_{\Delta x}\left(\eta_{1}, q_{1}\right)\right\}_{\Delta x>0} \in \mathcal{M}_{\mathrm{loc}}\left(\Pi_{T}\right) .
$$

Using (5.16), (5.17) in tandem with Lemma 2.4, we get the compactness of $\left\{\mathcal{E}_{\Delta x}^{1,1,1,1}(\eta, q)\right\}_{\Delta x>0}$ in $H_{\text {loc }}^{-1}\left(\Pi_{T}\right)$, for $(\eta, q):=\left(\eta_{1}, q_{1}\right)$.

Estimate of $\mathcal{E}_{\Delta x}^{1,1,1,1}$ for $(\eta, q):=\left(\eta_{2}, q_{2}\right):$ We recall that

$$
\eta_{2}(k, u)=k(f(u)-f(c)), \quad q_{2}(k, u)=\int_{c}^{u}\left(k f^{\prime}(\xi)\right)^{2} d \xi
$$

hence,

$$
\frac{\partial}{\partial u} \eta_{2}(k, u)=k f^{\prime}(u), \quad \frac{\partial}{\partial u} q_{2}(k, u)=k^{2} f^{\prime}(u)^{2} .
$$

This implies that

$$
\begin{aligned}
-\frac{\partial}{\partial u} \eta_{2}\left(k_{j-\frac{1}{2}}, u_{j}^{n}\right) k_{j-\frac{1}{2}} & D_{-} \hat{f}_{j+\frac{1}{2}}^{n}+\frac{1}{\Delta x}\left[q_{2}\left(k_{j-\frac{1}{2}}, u_{j}^{n}\right)-q_{2}\left(k_{j-\frac{1}{2}}, u_{j-1}^{n}\right)\right] \\
& =-k_{j-\frac{1}{2}}^{2} f^{\prime}\left(u_{j}^{n}\right) D_{-} \hat{f}_{j+\frac{1}{2}}^{n}+\frac{1}{\Delta x}\left[q_{2}\left(k_{j-\frac{1}{2}}, u_{j}^{n}\right)-q_{2}\left(k_{j-\frac{1}{2}}, u_{j-1}^{n}\right)\right] .
\end{aligned}
$$

Again, by Taylor's theorem

$$
\begin{aligned}
q_{2}\left(k_{j-\frac{1}{2}}, u_{j}^{n}\right)-q_{2}\left(k_{j-\frac{1}{2}}, u_{j-1}^{n}\right) & =\left(u_{j}^{n}-u_{j-1}^{n}\right) \frac{\partial}{\partial u} q_{2}\left(k_{j-\frac{1}{2}}, u_{j}^{n}\right)+\frac{1}{2} \frac{\partial^{2}}{\partial u^{2}} q_{2}\left(k_{j-\frac{1}{2}}, \theta_{j-\frac{1}{2}}^{n}\right)\left(u_{j}^{n}-u_{j-1}^{n}\right)^{2} \\
& =k_{j-\frac{1}{2}}^{2} f^{\prime}\left(u_{j}^{n}\right)^{2}\left(u_{j}^{n}-u_{j-1}^{n}\right)+\frac{1}{2} \frac{\partial^{2}}{\partial u^{2}} q_{2}\left(k_{j-\frac{1}{2}}, \theta_{j-\frac{1}{2}}^{n}\right)\left(u_{j}^{n}-u_{j-1}^{n}\right)^{2} \\
& =k_{j-\frac{1}{2}}^{2} f^{\prime}\left(u_{j}^{n}\right)\left(f\left(u_{j}^{n}\right)-f\left(u_{j-1}^{n}\right)\right)+\frac{1}{2} \frac{\partial^{2}}{\partial u^{2}} q_{2}\left(k_{j-\frac{1}{2}}, \theta_{j-\frac{1}{2}}^{n}\right)\left(u_{j}^{n}-u_{j-1}^{n}\right)^{2},
\end{aligned}
$$


where $\theta_{j-\frac{1}{2}}^{n}$ lies between $u_{j}^{n}$ and $u_{j-1}^{n}$. Thus

$$
\begin{aligned}
-\frac{\partial}{\partial u} \eta_{2}\left(k_{j-\frac{1}{2}}, u_{j}^{n}\right) k_{j-\frac{1}{2}} & D_{-} \hat{f}_{j+\frac{1}{2}}^{n}+\frac{1}{\Delta x}\left[q_{2}\left(k_{j-\frac{1}{2}}, u_{j}^{n}\right)-q_{2}\left(k_{j-\frac{1}{2}}, u_{j-1}^{n}\right)\right] \\
& =k_{j-\frac{1}{2}}^{2} f^{\prime}\left(u_{j}^{n}\right) D_{-}\left(f\left(u_{j}^{n}\right)-\hat{f}_{j+\frac{1}{2}}^{n}\right)+\frac{1}{2 \Delta x} \frac{\partial^{2}}{\partial u^{2}} q_{2}\left(k_{j-\frac{1}{2}}, \theta_{j-\frac{1}{2}}^{n}\right)\left(u_{j}^{n}-u_{j-1}^{n}\right)^{2} .
\end{aligned}
$$

Therefore

$$
\begin{aligned}
\left\langle\mathcal{E}_{\Delta x}^{1,1,1,1}\left(\eta_{2}, q_{2}\right), \varphi\right\rangle= & \underbrace{\sum_{n=0}^{N-1} \int_{t^{n}}^{t^{n+1}} \sum_{j}\left(k_{j-\frac{1}{2}}^{2} f^{\prime}\left(u_{j}^{n}\right) D_{-}\left(f\left(u_{j}^{n}\right)-\hat{f}_{j+\frac{1}{2}}^{n}\right)\right) \Phi_{j} \mathrm{~d} t}_{\left\langle\mathcal{Q}_{\Delta x}^{1}\left(\eta_{2}, q_{2}\right), \varphi\right\rangle} \\
& +\underbrace{\sum_{n=0}^{N-1} \int_{t^{n}}^{t^{n+1}} \sum_{j} \frac{1}{2 \Delta x} \frac{\partial^{2}}{\partial u^{2}} q_{2}\left(k_{j-\frac{1}{2}}, \theta_{j-\frac{1}{2}}^{n}\right)\left(u_{j}^{n}-u_{j-1}^{n}\right)^{2} \Phi_{j} \mathrm{~d} t}_{\left\langle\mathcal{Q}_{\Delta x}^{2}\left(\eta_{2}, q_{2}\right), \varphi\right\rangle} .
\end{aligned}
$$

Making use of the a priori estimate(4.3b), we conclude that

$$
\left\langle\mathcal{Q}_{\Delta x}^{2}\left(\eta_{2}, q_{2}\right), \varphi\right\rangle:=\sum_{n=0}^{N-1} \int_{t^{n}}^{t^{n+1}} \sum_{j} \frac{1}{2 \Delta x} \frac{\partial^{2}}{\partial u^{2}} q_{2}\left(k_{j-\frac{1}{2}}, \theta_{j-\frac{1}{2}}^{n}\right)\left(u_{j}^{n}-u_{j-1}^{n}\right)^{2} \Phi_{j} \mathrm{~d} t \leq C\|\varphi\|_{\infty} .
$$

Hence

$$
\left\{\mathcal{Q}_{\Delta x}^{2}\left(\eta_{2}, q_{2}\right)\right\}_{\Delta x>0} \in \mathcal{M}_{\mathrm{loc}}\left(\Pi_{T}\right) .
$$

On the other hand, using summation by parts, we can estimate the other term as follows

$$
\begin{aligned}
\left\langle\mathcal{Q}_{\Delta x}^{1}\left(\eta_{2}, q_{2}\right), \varphi\right\rangle:=\sum_{n=0}^{N-1} \int_{t^{n}}^{t^{n+1}} \sum_{j}\left(k_{j-\frac{1}{2}}^{2} f^{\prime}\left(u_{j}^{n}\right) D_{-}\left(f\left(u_{j}^{n}\right)-\hat{f}_{j+\frac{1}{2}}^{n}\right)\right) \Phi_{j} \mathrm{~d} t & -\sum_{n=0}^{N-1} \int_{t^{n}}^{t^{n+1}} \sum_{j} D_{+}\left(k_{j-\frac{1}{2}}^{2} f^{\prime}\left(u_{j}^{n}\right) \Phi_{j}\right)\left(f\left(u_{j}^{n}\right)-\hat{f}_{j+\frac{1}{2}}^{n}\right) \mathrm{d} t \\
= & -\underbrace{\sum_{n=0}^{N-1} \int_{t^{n}}^{t^{n+1}} \sum_{j}\left(f\left(u_{j}^{n}\right)-\hat{f}_{j+\frac{1}{2}}^{n}\right)\left(D_{+} k_{j-\frac{1}{2}}^{2}\right) f^{\prime}\left(u_{j+1}^{n}\right) \Phi_{j+1} \mathrm{~d} t}_{\left\langle\mathcal{X}_{\Delta x}\left(\eta_{2}, q_{2}\right), \varphi\right\rangle} \\
& -\underbrace{\sum_{n=0}^{N-1} \int_{t^{n}}^{t^{n+1}} \sum_{j}\left(f\left(u_{j}^{n}\right)-\hat{f}_{j+\frac{1}{2}}^{n}\right) k_{j-\frac{1}{2}}^{2}\left(D_{+} f^{\prime}\left(u_{j}^{n}\right)\right) \Phi_{j+1} \mathrm{~d} t}_{\left\langle\mathcal{Y}_{\Delta x}\left(\eta_{2}, q_{2}\right), \varphi\right\rangle} \\
& -\underbrace{\left.\sum_{n=0}^{N-1} \int_{t^{n}}^{t^{n+1}} \sum_{j}\left(f\left(u_{j}^{n}\right)-\hat{f}_{j+\frac{1}{2}}^{n}\right) k_{j-\frac{1}{2}}^{2} f^{\prime}\left(u_{j}^{n}\right)\right) D_{-}\left(\Phi_{j}\right) \mathrm{d} t}_{\left\langle\mathcal{Z}_{\Delta x}\left(\eta_{2}, q_{2}\right), \varphi\right\rangle} .
\end{aligned}
$$


Using essentially the same type of arguments as before, we can deal with the above terms and prove the following estimates:

$$
\begin{aligned}
& \left|\left\langle\mathcal{X}_{\Delta x}\left(\eta_{2}, q_{2}\right), \varphi\right\rangle\right| \leq C\|\varphi\|_{\infty}|k|_{B V}\|k\|_{\infty}, \\
& \left|\left\langle\mathcal{Y}_{\Delta x}\left(\eta_{2}, q_{2}\right), \varphi\right\rangle\right| \leq C\|\varphi\|_{\infty}\|k\|_{\infty}, \\
& \left|\left\langle\mathcal{Z}_{\Delta x}\left(\eta_{2}, q_{2}\right), \varphi\right\rangle\right| \leq C(\Delta x)^{1 / 2}\|\varphi\|_{H^{1}}\|k\|_{\infty},
\end{aligned}
$$

with the help of (5.15), and the a priori estimates (4.3b), and (4.3c). This implies that

$$
\left\{\mathcal{X}_{\Delta x}\left(\eta_{2}, q_{2}\right)\right\}_{\Delta x>0},\left\{\mathcal{Y}_{\Delta x}\left(\eta_{2}, q_{2}\right)\right\}_{\Delta x>0} \in \mathcal{M}_{\text {loc }}\left(\Pi_{T}\right)
$$

and

$$
\left\{\mathcal{Z}_{\Delta x}\left(\eta_{2}, q_{2}\right)\right\}_{\Delta x>0} \text { is compact in } H_{\text {loc }}^{-1}\left(\Pi_{T}\right) .
$$

We use Lemma 2.4 along with $(5.18)-(5.20)$ to conclude the compactness of $\left\{\mathcal{E}_{\Delta x}^{1,1,1,1}(\eta, q)\right\}_{\Delta x>0}$ in $H_{\text {loc }}^{-1}\left(\Pi_{T}\right)$, for $(\eta, q):=\left(\eta_{2}, q_{2}\right)$ as well.

Finally, the compactness of $\left\{\mathcal{E}_{\Delta x}^{1,1,1,1}(\eta, q)\right\}_{\Delta x>0}$ along with (5.14) ensures the compactness of $\left\{\mathcal{E}_{\Delta x}^{1,1,1}(\eta, q)\right\}_{\Delta x>0}$ in $H_{\text {loc }}^{-1}\left(\Pi_{T}\right)$.

Next, we tackle the term $\mathcal{E}_{\Delta x}^{1,1,2}(\eta, q)$.

\section{Lemma 5.5.}

$$
\left\{\mathcal{E}_{\Delta x}^{1,1,2}(\eta, q)\right\}_{\Delta x>0} \text { is compact in } H_{\mathrm{loc}}^{-1}\left(\Pi_{T}\right) .
$$

Proof. We first recall the expression for $\left\langle\mathcal{E}_{\Delta x}^{1,1,2}(\eta, q), \varphi\right\rangle$

$$
\left\langle\mathcal{E}_{\Delta x}^{1,1,2}(\eta, q), \varphi\right\rangle=\beta \sum_{j} \sum_{n=0}^{N-1} \int_{t^{n}}^{t^{n+1}} \int_{I_{j}} \eta_{u}\left(k_{j-\frac{1}{2}}, u_{j}^{n}\right) \Delta x\left(D_{+} D_{-} u_{j}^{n}\right) \varphi \mathrm{d} x \mathrm{~d} t .
$$

Using summation-by-parts formula, we can write

$$
\begin{aligned}
\left\langle\mathcal{E}_{\Delta x}^{1,1,2}(\eta, q), \varphi\right\rangle= & -\underbrace{\beta \sum_{n=0}^{N-1} \int_{t^{n}}^{t^{n+1}} \sum_{j} \Delta x D_{-} u_{j}^{n} D_{-}\left(\frac{\partial}{\partial u} \eta\left(k_{j-\frac{1}{2}}, u_{j}^{n}\right)\right) \Phi_{j-1} \mathrm{~d} t}_{\left\langle\mathcal{E}_{\Delta x}^{1,1,2,1}(\eta, q), \varphi\right\rangle} \\
& -\underbrace{\beta \sum_{n=0}^{N-1} \int_{t^{n}}^{t^{n+1}} \sum_{j} \Delta x D_{-} u_{j}^{n} \frac{\partial}{\partial u} \eta\left(k_{j-\frac{1}{2}}, u_{j}^{n}\right) D_{-} \Phi_{j} \mathrm{~d} t}_{\left\langle\mathcal{E}_{\Delta x}^{1,1,2,2}(\eta, q), \varphi\right\rangle} .
\end{aligned}
$$

Now we write $\mathcal{E}_{\Delta x}^{1,1,2,1}(\eta, q)$ as

$$
\begin{gathered}
\left\langle\mathcal{E}_{\Delta x}^{1,1,2,1}(\eta, q), \varphi\right\rangle=-\underbrace{\beta \sum_{n=0}^{N-1} \int_{t^{n}}^{t^{n+1}} \Delta x \sum_{j} D_{-} u_{j}^{n} \frac{\eta_{u}\left(k_{j-\frac{1}{2}}, u_{j}^{n}\right)-\eta_{u}\left(k_{j-\frac{1}{2}}, u_{j-1}^{n}\right)}{\Delta x} \Phi_{j-1} \mathrm{~d} t}_{\left\langle\mathcal{A}_{\Delta x}(\eta, q), \varphi\right\rangle} \\
-\underbrace{\beta \sum_{n=0}^{N-1} \int_{t^{n}}^{t^{n+1}} \sum_{j} \Delta x D_{-} u_{j}^{n} \frac{\eta_{u}\left(k_{j-\frac{1}{2}}, u_{j-1}^{n}\right)-\eta_{u}\left(k_{j-\frac{3}{2}}, u_{j-1}^{n}\right)}{\Delta x} \Phi_{j-1} \mathrm{~d} t}_{\left\langle\mathcal{B}_{\Delta x}(\eta, q), \varphi\right\rangle} .
\end{gathered}
$$


Since $\eta^{\prime \prime}$ is bounded, we can estimate $\mathcal{B}_{\Delta x}(\eta, q)$ with the help of Cauchy-Schwartz inequality (multiple times) and $a$ priori estimate (4.3b)

$$
\begin{aligned}
\left|\left\langle\mathcal{B}_{\Delta x}(\eta, q), \varphi\right\rangle\right| \leq C \beta\|\varphi\|_{\infty} \sum_{n=0}^{N-1} \int_{t^{n}}^{t^{n+1}} \sum_{j} \Delta x\left|D_{-} u_{j}^{n}\right|\left|k_{j-\frac{1}{2}}-k_{j-\frac{3}{2}}\right| \mathrm{d} t \\
\quad \leq C \beta\|\varphi\|_{\infty} \sum_{n=0}^{N-1} \int_{t^{n}}^{t^{n+1}}\left(\sum_{j}(\Delta x)^{2}\left(D_{-} u_{j}^{n}\right)^{2}\right)^{1 / 2}\left(\sum_{j}\left|k_{j-\frac{1}{2}}-k_{j-\frac{3}{2}}\right|^{2}\right)^{1 / 2} \mathrm{~d} t \\
\leq C \beta\|\varphi\|_{\infty} \sum_{n=0}^{N-1}\left(\int_{t^{n}}^{t^{n+1}} \sum_{j}(\Delta x)^{2}\left(D_{-} u_{j}^{n}\right)^{2} \mathrm{~d} t\right)^{1 / 2}\left(\int_{t^{n}}^{t^{n+1}} \sum_{j}\left|k_{j-\frac{1}{2}}-k_{j-\frac{3}{2}}\right|^{2} \mathrm{~d} t\right)^{1 / 2} \\
\leq C \beta\|\varphi\|_{\infty}\left(\sum_{n=0}^{N-1} \int_{t^{n}}^{t^{n+1}} \sum_{j}(\Delta x)^{2}\left(D_{-} u_{j}^{n}\right)^{2} \mathrm{~d} t\right)^{1 / 2} T^{1 / 2}\left(\sum_{j}\left|k_{j-\frac{1}{2}}-k_{j-\frac{3}{2}}\right|^{2}\right)^{1 / 2} \\
\leq C \beta\|\varphi\|_{\infty}|k|_{B V} .
\end{aligned}
$$

Similarly, we can obtain the following estimate for $\mathcal{A}_{\Delta x}(\eta, q)$,

$$
\left|\left\langle\mathcal{A}_{\Delta x}(\eta, q), \varphi\right\rangle\right| \leq \beta\|\varphi\|_{\infty} \Delta x \sum_{n=0}^{N-1} \int_{t^{n}}^{t^{n+1}}\left\|D_{-} u^{n}\right\|^{2} \mathrm{~d} t \leq \beta C\|\varphi\|_{\infty} .
$$

Hence

$$
\left\{\mathcal{A}_{\Delta x}(\eta, q)\right\}_{\Delta x>0},\left\{\mathcal{B}_{\Delta x}(\eta, q)\right\}_{\Delta x>0} \in \mathcal{M}_{\mathrm{loc}}\left(\Pi_{T}\right) \quad \Longrightarrow \quad\left\{\mathcal{E}_{\Delta x}^{1,1,2,1}(\eta, q)\right\}_{\Delta x>0} \in \mathcal{M}_{\mathrm{loc}}\left(\Pi_{T}\right) .
$$

Next, consider $\mathcal{E}_{\Delta x}^{1,1,2,2}(\eta, q)$. Assuming that $\eta^{\prime}$ is bounded, and using Cauchy-Schwartz inequality along with the a priori estimate (4.3b) and the estimate (5.15), we obtain

$$
\begin{aligned}
\left\langle\mathcal{E}_{\Delta x}^{1,1,2,2}(\eta, q), \varphi\right\rangle & =-\beta \sum_{n=0}^{N-1} \int_{t^{n}}^{t^{n+1}} \sum_{j} \Delta x D_{-} u_{j}^{n} \frac{\partial}{\partial u} \eta\left(k_{j-\frac{1}{2}}, u_{j}^{n}\right) D_{-} \Phi_{j} \mathrm{~d} t \\
& \leq \beta C \sum_{n=0}^{N-1} \int_{t^{n}}^{t^{n+1}} \sum_{j} \Delta x\left|D_{-} u_{j}^{n}\right|\left|D_{-} \Phi_{j}\right| \mathrm{d} t \\
& \leq \beta C\left(\Delta x^{2} \sum_{n=0}^{N-1} \int_{t^{n}}^{t^{n+1}}\left\|D_{-} u^{n}\right\|^{2} \mathrm{~d} t\right)^{1 / 2}\left(\sum_{n=0}^{N-1} \int_{t^{n}}^{t^{n+1}} 4 \Delta x\left\|\varphi_{x}\right\|^{2}\right)^{1 / 2} \\
& \leq \beta C(\Delta x)^{1 / 2}\|\varphi\|_{H^{1}\left(\Pi_{T}\right)} .
\end{aligned}
$$

Therefore,

$$
\left\{\mathcal{E}_{\Delta x}^{1,1,2,2}(\eta, q)\right\}_{\Delta x>0} \text { is compact in } H_{\text {loc }}^{-1}\left(\Pi_{T}\right)
$$

Thus, (5.22), and (5.23) ensure the compactness of $\left\{\mathcal{E}_{\Delta x}^{1,1,2}(\eta, q)\right\}_{\Delta x>0}$.

Next, we focus on $\mathcal{E}_{\Delta x}^{1,1,3}(\eta, q)$. 


\section{Lemma 5.6.}

$$
\left\{\mathcal{E}_{\Delta x}^{1,1,3}(\eta, q)\right\}_{\Delta x>0} \text { is compact in } H_{\mathrm{loc}}^{-1}\left(\Pi_{T}\right) .
$$

Proof. Recall that

$$
\left\langle\mathcal{E}_{\Delta x}^{1,1,3}(\eta, q), \varphi\right\rangle=\gamma \sum_{j} \sum_{n=0}^{N-1} \int_{t^{n}}^{t^{n+1}} \int_{I_{j}} \eta_{u}\left(k_{j-\frac{1}{2}}, u_{j}^{n}\right) \mu(\Delta x)\left(D_{+}^{t} D_{+} D_{-} u_{j}^{n}\right) \varphi \mathrm{d} x \mathrm{~d} t .
$$

Making use of the summation-by-parts formula and discrete Libnitz rule, we rewrite

$$
\begin{aligned}
\left\langle\mathcal{E}_{\Delta x}^{1,1,3}(\eta, q), \varphi\right\rangle= & -\underbrace{\gamma \sum_{n=0}^{N-1} \int_{t^{n}}^{t^{n+1}} \sum_{j} \mu(\Delta x) D_{+}^{t} D_{-} u_{j}^{n} D_{-}\left(\frac{\partial}{\partial u} \eta\left(k_{j-\frac{1}{2}}, u_{j}^{n}\right)\right) \Phi_{j-1}}_{\left\langle\mathcal{C}_{\Delta x}(\eta, q), \varphi\right\rangle} \mathrm{d} t \\
& -\underbrace{\gamma \sum_{n=0}^{N-1} \int_{t^{n}}^{t^{n+1}} \sum_{j} \mu(\Delta x) D_{+}^{t} D_{-}\left(u_{j}^{n}\right) \frac{\partial}{\partial u} \eta\left(k_{j-\frac{1}{2}}, u_{j}^{n}\right) D_{-} \Phi_{j} \mathrm{~d} t}_{\left\langle\mathcal{D}_{\Delta x}(\eta, q), \varphi\right\rangle} .
\end{aligned}
$$

Again, making use of the a priori estimate (4.3d) reveals that the term $\mathcal{D}_{\Delta x}(\eta, q)$ can be estimated as

$$
\left|\left\langle\mathcal{D}_{\Delta x}(\eta, q), \varphi\right\rangle\right| \leq \gamma C(\Delta x)^{\frac{1}{2}}\left(\mu(\Delta x) \Delta x \Delta t \sum_{n=0}^{N-1}\left\|D_{+}^{t} D_{-} u^{n}\right\|^{2}\right)^{1 / 2}\|\varphi\|_{H^{1}\left(\Pi_{T}\right)} \leq \gamma C(\Delta x)^{\frac{1}{2}}\|\varphi\|_{H^{1}\left(\Pi_{T}\right)},
$$

so that

$$
\left\{\mathcal{D}_{\Delta x}(\eta, q)\right\}_{\Delta x>0} \text { is compact in } H_{\mathrm{loc}}^{-1}\left(\Pi_{T}\right) .
$$

On the other hand, to estimate $\mathcal{C}_{\Delta x}(\eta, q)$ term, we first split that term as

$$
\begin{aligned}
\left\langle\mathcal{C}_{\Delta x}(\eta, q), \varphi\right\rangle= & -\underbrace{\gamma \sum_{n, j} \int_{t^{n}}^{t^{n+1}} \mu(\Delta x) D_{+}^{t} D_{-} u_{j}^{n} \frac{\eta_{u}\left(k_{j-\frac{1}{2}}, u_{j}^{n}\right)-\eta_{u}\left(k_{j-\frac{1}{2}}, u_{j-1}^{n}\right)}{\Delta x} \Phi_{j-1} \mathrm{~d} t}_{\left\langle\mathcal{G}_{\Delta x}(\eta, q), \varphi\right\rangle} \\
& -\underbrace{\gamma \sum_{n, j} \int_{t^{n}}^{t^{n+1}} \mu(\Delta x) D_{+}^{t} D_{-} u_{j}^{n} \frac{\eta_{u}\left(k_{j-\frac{1}{2}}, u_{j-1}^{n}\right)-\eta_{u}\left(k_{j-\frac{3}{2}}, u_{j-1}^{n}\right)}{\Delta x} \Phi_{j-1} \mathrm{~d} t}_{\left\langle\mathcal{F}_{\Delta x}(\eta, q), \varphi\right\rangle} .
\end{aligned}
$$

A similar type of argument, as used before, can be used to deal with the terms $\mathcal{G}_{\Delta x}$ and $\mathcal{F}_{\Delta x}$, with the help of the a priori estimate $(4.3 \mathrm{~d})$, returns

$$
\begin{aligned}
& \left|\left\langle\mathcal{G}_{\Delta x}(\eta, q), \varphi\right\rangle\right| \\
& \leq 2 \gamma\|\varphi\|_{L^{\infty}\left(\Pi_{T}\right)}\left(\mu(\Delta x) \sum_{n=0}^{N} \Delta t \Delta x\left\|D_{+}^{t} D_{-} u^{n}\right\|^{2}\right)^{1 / 2}\left(\sum_{n=0}^{N} \Delta t \Delta x\left\|D_{-} u^{n}\right\|^{2}\right)^{1 / 2} \leq \gamma C\|\varphi\|_{L^{\infty}\left(\Pi_{T}\right)},
\end{aligned}
$$

and

$$
\left|\left\langle\mathcal{F}_{\Delta x}(\eta, q), \varphi\right\rangle\right| \leq \gamma \operatorname{BV}(k)\|\varphi\|_{L^{\infty}\left(\Pi_{T}\right)}\left(\mu(\Delta x) \Delta x \Delta t \sum_{n=0}^{N-1}\left\|D_{+}^{t} D_{-} u^{n}\right\|^{2}\right)^{1 / 2} \leq \gamma C\|\varphi\|_{L^{\infty}\left(\Pi_{T}\right)} .
$$


This implies that

$$
\left\{\mathcal{G}_{\Delta x}(\eta, q)\right\}_{\Delta x>0},\left\{\mathcal{F}_{\Delta x}(\eta, q)\right\}_{\Delta x>0} \in \mathcal{M}_{\text {loc }}\left(\Pi_{T}\right) .
$$

Therefore, in view of the Lemma 2.4 along with (5.25), and (5.26), we reach at the conclusion

$$
\left\{\mathcal{E}_{\Delta x}^{1,1,3}(\eta, q)\right\}_{\Delta x>0} \text { is compact in } H_{\text {loc }}^{-1}\left(\Pi_{T}\right) .
$$

Making use of Lemma 5.4, 5.5 and 5.6, we can finally prove the following.

\section{Lemma 5.7.}

$$
\left\{\mathcal{E}_{\Delta x}^{1,1}(\eta, q)\right\}_{\Delta x>0} \text { is compact in } H_{\mathrm{loc}}^{-1}\left(\Pi_{T}\right)
$$

Proof. We have already shown that $\left\{\mathcal{E}_{\Delta x}^{1,1,1}(\eta, q)\right\}_{\Delta x>0},\left\{\mathcal{E}_{\Delta x}^{1,1,2}(\eta, q)\right\}_{\Delta x>0}$ and $\left\{\mathcal{E}_{\Delta x}^{1,1,3}(\eta, q)\right\}_{\Delta x>0}$ are compact $H^{-1}\left(\Pi_{T}\right)$. Thus, we only need to find an estimate for $\mathcal{E}_{\Delta x}^{1,1,4}(\eta, q)$. Recall that,

$$
\left\langle\mathcal{E}_{\Delta x}^{1,1,4}(\eta, q), \varphi\right\rangle=\sum_{j} \sum_{n=0}^{N-1} \int_{t^{n}}^{t^{n+1}} \int_{I_{j}} \frac{\Delta t}{2} \eta_{u u}\left(k_{j-\frac{1}{2}}, \theta_{j}^{n+\frac{1}{2}}\right)\left(D_{+}^{t} u_{j}^{n}\right)^{2} \varphi \mathrm{d} x \mathrm{~d} t .
$$

Using the a priori bound (4.3b), we have

$$
\left|\left\langle\mathcal{E}_{\Delta x}^{1,1,4}(\eta, q), \varphi\right\rangle\right| \leq C\|\varphi\|_{\infty}\left(\sum_{n=0}^{N} \Delta t \Delta x\left\|D_{+}^{t} u^{n}\right\|^{2}\right) \leq C\|\varphi\|_{\infty},
$$

so that

$$
\left\{\mathcal{E}_{\Delta x}^{1,1,4}(\eta, q)\right\}_{\Delta x>0} \in \mathcal{M}_{\mathrm{loc}}\left(\Pi_{T}\right)
$$

An application of Lemma 2.4 allows us to conclude (5.28), thus proving the lemma.

Combining Lemma 5.3 and 5.7 ensures that

$$
\left\{\mathcal{E}_{\Delta x}^{1}(\eta, q)\right\}_{\Delta x>0} \text { is compact in } H_{\mathrm{loc}}^{-1}\left(\Pi_{T}\right) .
$$

Thus, Lemma 5.2 and (5.30) justify the assumptions we had made in the proof of Lemma 5.1.

Now we are in a position to state the "convergence theorem" which guarantees the convergence of approximate solutions $\left\{u_{\Delta x}\right\}_{\Delta x>0}$, generated by the scheme (3.2), to a weak solution of (1.4). The following theorem can also be viewed as a modified version of the classical Lax-Wendroff theorem (for more details, consult the monograph by LeVeque [22]).

Theorem 5.8. Let $u_{\Delta x}$ be a sequence of approximations generated via the scheme $(3.2)$ with $\mu(\Delta x)=\mathcal{O}\left(\Delta x^{2}\right)$. Then there exists a function $u \in L^{\infty}\left([0, T] ; L_{\text {loc }}^{1}(\mathbb{R})\right)$ and a subsequence of $\{\Delta x\}$ (not relabeled) such that $u_{\Delta x} \mapsto u$ as $\Delta x \downarrow 0$. Moreover, the function $u$ is a weak solution to (1.4). 
Proof. The strong convergence of $u_{\Delta x}$ to a function $u$ immediately follows from the Theorem 2.3 and the Lemma 5.1. It remains to show that $u$ is a weak solution. Let $\psi \in C_{0}^{\infty}(\mathbb{R} \times[0, T))$ be any test function and denote $\psi_{j}^{n}=\psi\left(x_{j}, t^{n}\right)$. Multiplying the scheme (3.2) by $\Delta x \Delta t \psi_{j}^{n}$, and subsequently suming over all $j$ and $n$ yields

$$
\begin{aligned}
\Delta x \Delta t \sum_{j} \sum_{n} \psi_{j}^{n} D_{+}^{t} u_{j}^{n}+\Delta x \Delta t \sum_{j} \sum_{n} \psi_{j}^{n} D_{-}\left(k_{j+\frac{1}{2}} \hat{f}_{j+\frac{1}{2}}\right) \\
=\beta \Delta x \Delta t \sum_{j} \sum_{n} \Delta x \psi_{j}^{n} D_{+} D_{-} u_{j}^{n}+\gamma \Delta x \Delta t \sum_{j} \sum_{n} \mu(\Delta x) \psi_{j}^{n} D_{+}^{t} D_{+} D_{-} u_{j}^{n} .
\end{aligned}
$$

By standard arguments, it is clear that

$$
\begin{aligned}
\Delta x \Delta t \sum_{j} \sum_{n} \psi_{j}^{n} D_{+}^{t} u_{j}^{n}= & -\Delta x \Delta t \sum_{j} \sum_{n} D_{-}^{t} \psi_{j}^{n} u_{j}^{n}-\Delta x \sum_{j} \psi_{j}^{0} u_{j}^{0} \\
& \mapsto-\int_{\mathbb{R}} \int_{0}^{T} u \psi_{t} \mathrm{~d} x \mathrm{~d} t-\int_{\mathbb{R}} \psi(x, 0) u_{0}(x) \mathrm{d} x, \text { as } \Delta x \downarrow 0 .
\end{aligned}
$$

Next, using the a priori bound (4.3b), we conclude

$$
\begin{aligned}
& \beta \Delta x \Delta t \sum_{j} \sum_{n} \Delta x \psi_{j}^{n} D_{+} D_{-} u_{j}^{n}=-\beta \Delta x \Delta t \sum_{j} \sum_{n} \Delta x D_{-} \psi_{j}^{n} D_{-} u_{j}^{n} \\
& \leq \beta \Delta x \Delta t \sum_{n}\left(\Delta x \sum_{j}\left(D_{-} u_{j}^{n}\right)^{2}\right)^{1 / 2}\left(\Delta x \sum_{j}\left(D_{-} \psi_{j}^{n}\right)^{2}\right)^{1 / 2} \\
& \leq \beta\left(\Delta x^{2} \Delta t \sum_{n} \sum_{j}\left(D_{-} u_{j}^{n}\right)^{2}\right)^{1 / 2}\left(\Delta x^{2} \Delta t \sum_{n} \sum_{j}\left(D_{-} \psi_{j}^{n}\right)^{2}\right)^{1 / 2} \\
& \leq \beta C(\Delta x)^{1 / 2}\|\psi\|_{L^{2}\left((0, T) ; H^{1}(\mathbb{R})\right)} \mapsto 0, \text { as } \Delta x \downarrow 0 .
\end{aligned}
$$

Repeated use of Cauchy-Schwartz inequality along with the help of a priori bound (4.3d) returns

$$
\begin{gathered}
\gamma \Delta x \Delta t \sum_{j} \sum_{n} \mu(\Delta x) \psi_{j}^{n} D_{+}^{t} D_{+} D_{-} u_{j}^{n} \\
\leq \gamma C \frac{\mu(\Delta x)^{1 / 2}}{\Delta x^{1 / 2}}\left(\sum_{j} \sum_{n} \mu(\Delta x) \Delta x^{2} \Delta t\left|D_{+}^{t} D_{-} u_{j}^{n}\right|^{2}\right)^{1 / 2}\left(\sum_{j} \sum_{n} \Delta x \Delta t\left|D_{-} \psi_{j}^{n}\right|^{2}\right)^{1 / 2} \\
\leq \gamma C \frac{\mu(\Delta x)^{1 / 2}}{\Delta x^{1 / 2}}\|\psi\|_{L^{2}\left((0, T) ; H^{1}(\mathbb{R})\right)} \mapsto 0, \text { as } \Delta x \downarrow 0 .
\end{gathered}
$$

Finally, a simple use of summation-by-parts formula implies

$$
\begin{aligned}
\Delta x \Delta t \sum_{j} \sum_{n} \psi_{j}^{n} D_{-} & \left(k_{j+\frac{1}{2}} \hat{f}_{j+\frac{1}{2}}^{n}\right)=-\Delta x \Delta t \sum_{j} \sum_{n} D_{+} \psi_{j}^{n} k_{j+\frac{1}{2}} \hat{f}_{j+\frac{1}{2}}^{n} \\
= & -\underbrace{\Delta t \sum_{j, n} \int_{I_{j}} D_{+} \psi_{j}^{n} k(x) f\left(u_{j}\right) \mathrm{d} x}_{\mathcal{E}_{\Delta x}^{1}}-\underbrace{\Delta t \sum_{j, n} \int_{I_{j}} D_{+} \psi_{j}^{n}\left(k_{j+\frac{1}{2}}-k(x)\right) \hat{f}_{j+\frac{1}{2}}^{n}}_{\mathcal{E}_{\Delta x}^{2}} \mathrm{~d} x \\
& -\underbrace{\Delta t \sum_{j} \sum_{I_{j}} \int_{I_{+}} D_{+} \psi_{j}^{n} k(x)\left(\hat{f}_{j+\frac{1}{2}}^{n}\right.}_{\mathcal{E}_{j}}-f\left(u_{j}\right)) \mathrm{d} x
\end{aligned}
$$


where, again, a standard argument reveals that

$$
\mathcal{E}_{\Delta x}^{1} \mapsto-\int_{\mathbb{R}} \int_{0}^{T} k(x) f(u) \psi_{x} \mathrm{~d} x \mathrm{~d} t, \text { as } \Delta x \downarrow 0 .
$$

Now observe that

$$
\sum_{j} \int_{I_{j}}\left|k(x)-k_{j+\frac{1}{2}}\right| \mathrm{d} x \leq \int_{\mathbb{R}}\left|k(x)-k_{\Delta x}(x)\right| \mathrm{d} x+\frac{\Delta x}{2} \int_{\mathbb{R}} \frac{|k(x+\Delta x / 2)-k(x)|}{\Delta x / 2} \mathrm{~d} x
$$

and consequently

$$
\sum_{j} \int_{I_{j}}\left|k(x)-k_{j+\frac{1}{2}}\right| \mathrm{d} x \mapsto 0 \text { as } \Delta x \downarrow 0 .
$$

Keeping this in mind, we find that

$$
\begin{aligned}
\mathcal{E}_{\Delta x}^{2} & =\Delta t \sum_{j, n} \int_{I_{j}} D_{+} \psi_{j}^{n}\left(k_{j+\frac{1}{2}}-k(x)\right) \hat{f}_{j+\frac{1}{2}}^{n} \mathrm{~d} x \\
& \leq M\|\psi\|_{L^{1}\left([0, T] ; L^{\infty}(\mathbb{R})\right)} \sum_{j} \int_{I_{j}}\left|k(x)-k_{j+\frac{1}{2}}\right| \mathrm{d} x \mapsto 0 \text { as } \Delta x \downarrow 0 .
\end{aligned}
$$

Finally, making use of the a priori bound (4.3b), the last term can be estimated as follows:

$$
\begin{aligned}
\mathcal{E}_{\Delta x}^{3} & =\Delta t \sum_{j} \sum_{n} \int_{I_{j}} D_{+} \psi_{j}^{n} k(x)\left(\hat{f}_{j+\frac{1}{2}}^{n}-f\left(u_{j}\right)\right) \mathrm{d} x \\
& \leq M(\Delta x)^{1 / 2}\|\psi\|_{L^{2}\left([0, T] ; H^{1}(\mathbb{R})\right)}\left(\Delta x^{2} \Delta t \sum_{j} \sum_{n}\left(D_{-} u_{j}^{n}\right)^{2}\right)^{1 / 2} \mapsto 0 \text { as } \Delta x \downarrow 0 .
\end{aligned}
$$

To sum up, we have proved that $u$ is a weak solution of (1.4), i.e.,

$$
\int_{\mathbb{R}} \int_{0}^{T}\left(\psi_{t} u+\psi_{x} k(x) f(u)\right) \mathrm{d} x \mathrm{~d} t+\int_{\mathbb{R}} \psi(x, 0) u_{0}(x) \mathrm{d} x=0 \text {, for all } \psi \in C_{0}^{\infty}(\mathbb{R} \times[0, T)) .
$$

\section{ENTROPY SOLUTION}

As we have already mentioned in Remark 3.2, we use the Engquist-Osher scheme to make the analysis more concrete, but our methods can easily be adapted to general monotone schemes. Drawing preliminary motivation from Karlsen et al. [14] and Towers [29], we first show that the limit solution satisfies the Kružkov entropy inequalities locally, away from the jumps in $k$. Then we proceed to show that the limit solution satisfies Kružkov type entropy inequalities when the test function has support which intersects one or more jumps in $k$, and finally we show that this implies Kružkov entropy solution.

To proceed further, we need some additional regularity assumptions on the discontinuous coefficient $k(x)$. We assume that $k(x)$ is piecewise Lipschitz continuous in $\mathbb{R}$ with finitely many jumps (in $k$ and $k^{\prime}$ ), located at $\xi_{1}, \xi_{2}, \ldots, \xi_{M}$. More specifically, we assume that there are finitely many Lipschitz continuous curves $\omega_{1}, \omega_{2}, \ldots, \omega_{M}$ such that $\xi_{i} \in \omega_{i}$, the union of which we denote by

$$
\bigcup_{i=1}^{M} \omega_{i}=\Omega
$$


For the sake of simplicity, we also assume that none of the curves intersect. The curves $\omega_{1}, \omega_{2}, \ldots, \omega_{M}$ partition $\mathbb{R} \backslash \Omega$ into a finite union of open sets:

$$
\mathbb{R} \backslash \Omega=\mathcal{R}_{0} \cup \mathcal{R}_{1} \cup \ldots \mathcal{R}_{M},
$$

with the curve $\omega_{m}$ separating the sets $\mathcal{R}_{m-1}$ and $\mathcal{R}_{m}$. We will assume that

$$
k \in \operatorname{Lip}\left(\overline{\mathcal{R}}_{m}\right), \quad m=1,2, \ldots, M .
$$

With this assumption, $k$ has well-defined limits from the right and left along each of the curves $\omega_{m}, 1 \leq m \leq M$, and we denote these limits by $k_{m}^{ \pm}:=k\left(\xi_{m}^{ \pm}\right)$respectively.

To this end, we first establish the following entropy inequality for smooth entropy function $\eta$, to avoid complications arising from the discontinuity $\eta^{\prime}(u)=\operatorname{sign}(u-c)$ in Kružkov entropy.

Lemma 6.1. Let $(\eta, Q)$ be a convex entropy pair with $\eta$ being a $C^{2}$-function and $\eta^{\prime}(u)=Q^{\prime}(u) f^{\prime}(u)$. For the test function $\psi \geq 0$ with compact support in $t>0, x \in \mathbb{R} \backslash \Omega$, and every $c \in \mathbb{R}$, the following entropy inequality holds

$$
\int_{\mathbb{R}} \int_{0}^{T}\left(\eta(u) \psi_{t}+k Q(u) \psi_{x}\right) \mathrm{d} x \mathrm{~d} t+\int_{\mathbb{R}} \eta\left(u_{0}\right) \psi(x, 0) \mathrm{d} x-\int_{\mathbb{R}} \int_{0}^{T} k^{\prime}(x)\left(\eta^{\prime}(u) f(u)-Q(u)\right) \psi \mathrm{d} x \mathrm{~d} t \geq 0,
$$

provided $\mu(\Delta x)=\mathcal{O}\left(\Delta x^{2}\right)$.

Proof. We begin by rewriting the scheme (3.2) as

$$
u_{j}^{n+1}=w_{j}^{n}+\beta \Delta x \Delta t D_{+} D_{-} u_{j}^{n}+\gamma \Delta t \mu(\Delta x) D_{t}^{+} D_{+} D_{-} u_{j}^{n},
$$

with

$$
w_{j}^{n}=u_{j}^{n}-\lambda \Delta_{+}\left(k_{j-\frac{1}{2}} \hat{f}_{j-\frac{1}{2}}^{n}\right),
$$

where $\Delta_{+}$denote the undivided forward difference operator, and $\hat{f}_{j+\frac{1}{2}}^{n}$ is the EO flux corresponding to the flux function $f(u)$. Let $H_{j+\frac{1}{2}}$ be a entropy flux function, consistent with $Q(u)$. Then, in light of Karlsen et al. ([14], Lem. 4.1), it is evident that for any $c \in \mathbb{R}$

$$
\left|w_{j}^{n}-c\right| \leq\left|u_{j}^{n}-c\right|-\lambda\left(k_{j+\frac{1}{2}} H_{j+\frac{1}{2}}-k_{j-\frac{1}{2}} H_{j-\frac{1}{2}}\right)-\lambda \operatorname{sign}\left(w_{j}^{n}-c\right) f(c) \Delta_{+} k_{j-\frac{1}{2}} .
$$

To obtain a similar type inequality for a smooth entropy-entropy flux pair, we follow a classical approximation argument. For a rigorous proof, we refer to the paper by Karlsen et al. ([15], Lem. 5.7). In what follows, we have the following inequality:

$$
\eta\left(w_{j}^{n}\right) \leq \eta\left(u_{j}^{n}\right)-\lambda\left(k_{j+\frac{1}{2}} H_{j+\frac{1}{2}}-k_{j-\frac{1}{2}} H_{j-\frac{1}{2}}\right)+\lambda\left(Q\left(w_{j}^{n}\right)-\eta^{\prime}\left(w_{j}^{n}\right) f\left(w_{j}^{n}\right)\right) \Delta_{+} k_{j-\frac{1}{2}} .
$$

After rearranging terms, a discrete entropy inequality for the scheme results

$$
\eta_{j}^{n+1} \leq \eta_{j}^{n}+\left(\eta_{j}^{n+1}-\eta\left(w_{j}^{n}\right)\right)-\lambda \Delta_{+}\left(k_{j-\frac{1}{2}} H_{j-\frac{1}{2}}\right)+\lambda \xi_{j}^{n} \Delta_{+} k_{j-\frac{1}{2}} .
$$

where $\xi_{j}^{n}=Q\left(w_{j}^{n}\right)-\eta^{\prime}\left(w_{j}^{n}\right) f\left(w_{j}^{n}\right)$ and $\eta_{j}^{n}=\eta\left(u_{j}^{n}\right)$. Next, multiplying the above inequality by $\Delta x \Delta t \psi_{j}^{n}$ with $\psi_{j}^{n}=\psi\left(x_{j}, t_{n}\right)$, where $\psi$ smooth, non-negative test function $\psi$ with compact support in $(\mathbb{R} \backslash \Omega) \times[0, T)$ and using summation by parts yields

$$
\begin{aligned}
\Delta x \Delta t \sum_{j} \sum_{n} \psi_{j}^{n} D_{+}^{t} \eta_{j}^{n}-\Delta x \Delta t & \sum_{j} \sum_{n} k_{j-\frac{1}{2}} H_{j-\frac{1}{2}} D_{-} \psi_{j}^{n} \\
& -\Delta x \Delta t \sum_{j} \sum_{n} \psi_{j}^{n}\left(\xi_{j}^{n} D_{+} k_{j-\frac{1}{2}}+\left(\eta_{j}^{n+1}-\eta\left(w_{j}^{n}\right)\right) / \Delta t\right) \leq 0 .
\end{aligned}
$$


As before, a standard argument reveals that

$$
\begin{aligned}
\Delta x \Delta t \sum_{j} \sum_{n} \psi_{j}^{n} D_{+}^{t} \eta\left(u_{j}^{n}\right)= & -\Delta x \Delta t \sum_{j} \sum_{n} D_{-}^{t} \psi_{j}^{n} \eta\left(u_{j}^{n}\right)-\Delta x \sum_{j} \psi_{j}^{0} \eta\left(u_{j}^{0}\right) \\
& \mapsto-\int_{\mathbb{R}} \int_{0}^{T} \eta(u) \psi_{t} \mathrm{~d} x \mathrm{~d} t-\int_{\mathbb{R}} \psi(x, 0) \eta\left(u_{0}(x)\right) \mathrm{d} x, \text { as } \Delta x \downarrow 0 .
\end{aligned}
$$

Next, we rewrite

$$
\begin{aligned}
\Delta x \Delta t \sum_{j} \sum_{n} k_{j+\frac{1}{2}} H_{j+\frac{1}{2}} D_{-} \psi_{j}^{n}= & \underbrace{\Delta x \Delta t \sum_{j} \sum_{n} k_{j+\frac{1}{2}}\left(H_{j+\frac{1}{2}}-Q\left(u_{j}^{n}\right)\right) D_{-} \psi_{j}^{n}}_{\mathcal{E}_{\Delta x}^{1}} \\
& +\underbrace{\Delta x \Delta t \sum_{j} \sum_{n} k_{j+\frac{1}{2}} Q\left(u_{j}^{n}\right) D_{-} \psi_{j}^{n}}_{\mathcal{E}_{\Delta x}^{2}},
\end{aligned}
$$

where it is straightforward to conclude that

$$
\mathcal{E}_{\Delta x}^{2} \mapsto \int_{\mathbb{R}} \int_{0}^{T} k(x) Q(u) \psi_{x} \mathrm{~d} x \mathrm{~d} t, \text { as } \Delta x \downarrow 0,
$$

and the a priori estimate (4.3b) gives that

$$
\left|\mathcal{E}_{\Delta x}^{1}\right| \leq C(\Delta x)^{1 / 2}\left(\Delta x^{2} \Delta t \sum_{n, j}\left|D_{-} u_{j}^{n}\right|^{2}\right)^{1 / 2}\|\psi\|_{L^{2}\left([0, T] ; H^{1}(\mathbb{R})\right)} \mapsto 0 \text { as } \Delta x \downarrow 0 .
$$

To estimate the term involving $D_{+} k_{j-\frac{1}{2}}$, we split the term as follows

$$
\begin{aligned}
& \Delta x \Delta t \sum_{n} \sum_{j} \psi_{j}^{n} \xi_{j}^{n} D_{+} k_{j-\frac{1}{2}}=\Delta x \Delta t \sum_{n} \sum_{j}\left(Q\left(w_{j}^{n}\right)-\eta^{\prime}\left(w_{j}^{n}\right) f\left(w_{j}^{n}\right)\right) \psi_{j}^{n} D_{+} k_{j-\frac{1}{2}} \\
& =\underbrace{\Delta x \Delta t \sum_{n} \sum_{j}\left(Q\left(u_{j}^{n}\right)-\eta^{\prime}\left(u_{j}^{n}\right) f\left(u_{j}^{n}\right)\right) \psi_{j}^{n} D_{+} k_{j-\frac{1}{2}}}_{\mathcal{E}_{\Delta x}^{a}} \\
& +\underbrace{\Delta x \Delta t \sum_{n} \sum_{j}\left(Q\left(w_{j}^{n}\right)-Q\left(u_{j}^{n}\right)\right) \psi_{j}^{n} D_{+} k_{j-\frac{1}{2}}}_{\mathcal{E}_{\Delta x}^{b}} \\
& +\underbrace{\Delta x \Delta t \sum_{n} \sum_{j}\left(\eta^{\prime}\left(w_{j}^{n}\right) f\left(w_{j}^{n}\right)-\eta^{\prime}\left(u_{j}^{n}\right) f\left(u_{j}^{n}\right)\right) \psi_{j}^{n} D_{+} k_{j-\frac{1}{2}}}_{\mathcal{E}_{\Delta x}^{c}} .
\end{aligned}
$$

Again, a straightforward argument shows that

$$
\mathcal{E}_{\Delta x}^{a} \mapsto \int_{\mathbb{R}} \int_{0}^{T}\left(Q(u)-\eta^{\prime}(u) f(u)\right) k^{\prime}(x) \psi \mathrm{d} x \mathrm{~d} t, \text { as } \Delta x \downarrow 0 .
$$

For the other terms, we intend to show that

$$
\mathcal{E}_{\Delta x}^{b}, \mathcal{E}_{\Delta x}^{c} \mapsto 0 \text { as } \Delta x \downarrow 0 .
$$


To see this, first note that

$$
\begin{aligned}
\left|Q\left(w_{j}^{n}\right)-Q\left(u_{j}^{n}\right)\right| \leq C\left|w_{j}^{n}-u_{j}^{n}\right| & \leq C \lambda\left|k_{j+\frac{1}{2}} \hat{f}_{j+\frac{1}{2}}^{n}-k_{j-\frac{1}{2}} \hat{f}_{j-\frac{1}{2}}^{n}\right| \\
& \leq C \lambda\left(\left|k_{j+\frac{1}{2}}-k_{j-\frac{1}{2}}\right|+\left|u_{j+1}^{n}-u_{j}^{n}\right|+\left|u_{j-1}^{n}-u_{j}^{n}\right|\right) .
\end{aligned}
$$

Since $k(x)$ is Lipschitz continuous within the support of $\psi$, it is clear that

$$
\Delta x \Delta t \sum_{n} \sum_{j} \psi_{j}^{n}\left|k_{j+\frac{1}{2}}-k_{j-\frac{1}{2}}\right|\left|D_{+} k_{j-\frac{1}{2}}\right| \leq\|\psi\|_{\infty} T \Delta x\left\|k^{\prime}\right\|_{\infty}|k|_{B V} \mapsto 0 \text { as } \Delta x \downarrow 0,
$$

and

$$
\begin{aligned}
\Delta x \Delta t \sum_{n} \sum_{j} \psi_{j}^{n}\left|D_{+} k_{j-\frac{1}{2}}\right| \mid u_{j+1}^{n} & -u_{j}^{n} \mid \\
& \leq(\Delta x)^{1 / 2}\left(\Delta x^{2} \Delta t \sum_{n, j}\left|D_{-} u_{j}^{n}\right|^{2}\right)^{1 / 2}\left\|k^{\prime}\right\|_{\infty}\|\psi\|_{L^{2}\left([0, T] ; L^{2}(\mathbb{R})\right)} \mapsto 0 \text { as } \Delta x \downarrow 0 .
\end{aligned}
$$

This proves that $\mathcal{E}_{\Delta x}^{b} \mapsto 0$ as $\Delta x \mapsto 0$. A similar calculations show that $\mathcal{E}_{\Delta x}^{c} \mapsto 0$ as $\Delta x \mapsto 0$.

Finally, the last term can be estimated via Taylor series as follows

$$
\begin{aligned}
& \Delta x \Delta t \sum_{n} \sum_{j} \psi_{j}^{n}\left(\eta_{j}^{n+1}-\eta\left(w_{j}^{n}\right)\right) / \Delta t \\
= & \beta \Delta x \Delta t \sum_{j} \sum_{n} \psi_{j}^{n} \eta^{\prime}\left(u_{j}^{n}\right) \Delta x D_{+} D_{-} u_{j}^{n}+\gamma \Delta x \Delta t \sum_{j} \sum_{n} \psi_{j}^{n} \eta^{\prime}\left(u_{j}^{n}\right) \mu(\Delta x) D_{t}^{+} D_{+} D_{-} u_{j}^{n} \\
& -\Delta x \sum_{j, n} \lambda \eta^{\prime \prime}\left(\theta_{2}\right) \psi_{j}^{n} \Delta_{+}\left(k_{j-\frac{1}{2}} \hat{f}_{j-\frac{1}{2}}^{n}\right)\left(u_{j}^{n+1}-w_{j}^{n}\right)+\frac{1}{2} \Delta x \sum_{j, n} \psi_{j}^{n} \eta^{\prime \prime}\left(\theta_{1}\right)\left(u_{j}^{n+1}-w_{j}^{n}\right)^{2} \\
:= & \mathcal{Q}_{\Delta x}^{1}+\mathcal{Q}_{\Delta x}^{2}+\mathcal{Q}_{\Delta x}^{3}+\mathcal{Q}_{\Delta x}^{4} .
\end{aligned}
$$

For the first term $\mathcal{Q}_{\Delta x}^{1}$, using the discrete chain rule, we proceed as follows

$$
\begin{aligned}
\mathcal{Q}_{\Delta x}^{1}:=\beta \Delta x \Delta t \sum_{j} \sum_{n} \psi_{j}^{n} \eta^{\prime}\left(u_{j}^{n}\right) \Delta x D_{+} D_{-} u_{j}^{n}= & \underbrace{\beta \Delta x \Delta t \sum_{j} \sum_{n} \Delta x \psi_{j}^{n} D_{+}\left(\eta^{\prime}\left(u_{j}^{n}\right) D_{-} u_{j}^{n}\right)}_{\mathcal{E}_{\Delta x}^{3}} \\
& -\underbrace{\beta \Delta x \Delta t \sum_{j} \sum_{n} \Delta x \psi_{j}^{n} D_{+} u_{j}^{n} \eta^{\prime \prime}\left(\theta_{j}^{n}\right) D_{+} u_{j}^{n}}_{\mathcal{E}_{\Delta x}^{4}},
\end{aligned}
$$

where using the non-negativity of the test function $\psi$, we conclude that

$$
\mathcal{E}_{\Delta x}^{4} \geq 0
$$

Moreover, using the a priori bound (4.3b), we find

$$
\left|\mathcal{E}_{\Delta x}^{3}\right| \leq \beta C(\Delta x)^{1 / 2}\left(\Delta x^{2} \Delta t \sum_{j} \sum_{n}\left(D_{-} u_{j}^{n}\right)^{2}\right)^{1 / 2}\|\psi\|_{L^{2}\left([0, T] ; H^{1}(\mathbb{R})\right)} \mapsto 0 \text { as } \Delta x \downarrow 0 .
$$


For the next term $\mathcal{Q}_{\Delta x}^{2}$, we proceed as follows:

$$
\begin{aligned}
\mathcal{Q}_{\Delta x}^{2}:=\gamma \Delta x \Delta t \sum_{j} \sum_{n} \psi_{j}^{n} \eta^{\prime}\left(u_{j}^{n}\right) \mu(\Delta x) D_{t}^{+} D_{+} D_{-} u_{j}^{n}= & \underbrace{\gamma \Delta x \Delta t \sum_{j, n} \mu(\Delta x) \psi_{j}^{n} D_{+}\left(\eta^{\prime}\left(u_{j}^{n}\right) D_{t}^{+} D_{-} u_{j}^{n}\right)}_{\mathcal{E}_{\Delta x}^{5}} \\
& \underbrace{-\gamma \Delta x \Delta t \sum_{j} \sum_{n} \mu(\Delta x) \psi_{j}^{n} D_{t}^{+} D_{+} u_{j}^{n} \eta^{\prime \prime}\left(\theta_{j}^{n}\right) D_{+} u_{j}^{n}}_{\mathcal{E}_{\Delta x}^{6}} .
\end{aligned}
$$

Making use of the a priori bound (4.3d), we conclude

$$
\left|\mathcal{E}_{\Delta x}^{5}\right| \leq \gamma C \frac{\mu(\Delta x)^{1 / 2}}{\Delta x^{1 / 2}}\left(\mu(\Delta x) \Delta x^{2} \Delta t \sum_{j} \sum_{n}\left(D_{+}^{t} D_{-} u_{j}^{n}\right)^{2}\right)^{1 / 2}\|\psi\|_{L^{2}\left([0, T] ; H^{1}(\mathbb{R})\right)} \mapsto 0 \text { as } \Delta x \downarrow 0,
$$

and

$$
\begin{aligned}
\left|\mathcal{E}_{\Delta x}^{6}\right| & \leq \gamma C \frac{\mu(\Delta x)^{1 / 2}}{\Delta x}\left(\mu(\Delta x) \Delta x^{2} \Delta t \sum_{j} \sum_{n}\left(D_{+}^{t} D_{-} u_{j}^{n}\right)^{2}\right)^{1 / 2}\left(\Delta x^{2} \Delta t \sum_{j} \sum_{n}\left(D_{-} u_{j}^{n}\right)^{2}\right)^{1 / 2}\|\psi\|_{\infty} \\
& \mapsto 0 \text { as } \Delta x \downarrow 0 .
\end{aligned}
$$

Next, we turn our focus on the term $\mathcal{Q}_{\Delta x}^{3}$. In fact, we write

$$
\begin{aligned}
\mathcal{Q}_{\Delta x}^{3}:= & \Delta x \sum_{j} \sum_{n} \lambda \eta^{\prime \prime}\left(\theta_{2}\right) \psi_{j}^{n} \Delta_{+}\left(k_{j-\frac{1}{2}} \hat{f}_{j-\frac{1}{2}}^{n}\right)\left(u_{j}^{n+1}-w_{j}^{n}\right) \\
= & \underbrace{\beta \Delta \sum_{j} \sum_{n} \lambda \eta^{\prime \prime}\left(\theta_{2}\right) \psi_{j}^{n} \Delta_{+}\left(k_{j-\frac{1}{2}} \hat{f}_{j-\frac{1}{2}}^{n}\right) \Delta x \Delta t D_{+} D_{-} u_{j}^{n}}_{\mathcal{E}_{\Delta x}^{7}} \\
& +\underbrace{\gamma \Delta x \sum_{j} \sum_{n} \lambda \eta^{\prime \prime}\left(\theta_{2}\right) \psi_{j}^{n} \Delta_{+}\left(k_{j-\frac{1}{2}} \hat{f}_{j-\frac{1}{2}}^{n}\right) \Delta t \mu(\Delta x) D_{t}^{+} D_{+} D_{-} u_{j}^{n} .}_{\mathcal{E}_{\Delta x}^{8}} .
\end{aligned}
$$

Before we proceed, we recall that in accordance to Remark $4.3 \lambda=\zeta \mu(\Delta x) /(\Delta x)^{2}$, or in other words $\Delta t=$ $\zeta \mu(\Delta x) / \Delta x$. We also need to use the identity $\Delta x D_{+} D_{-} u_{j}^{n}=D_{+} u_{j}^{n}-D_{-} u_{j}^{n}$. Thus, using the discrete chain rule for the term $\Delta_{+}\left(k_{j-\frac{1}{2}} \hat{f}_{j-\frac{1}{2}}^{n}\right)$ and apply Cauchy-Schwartz inequality repeatedly, we obtain

$$
\left|\mathcal{E}_{\Delta x}^{7}\right| \leq \beta C \frac{\mu(\Delta x)}{\Delta x^{2}}\|\psi\|_{\infty}\left[|k|_{B V}\left(\Delta x^{2} \Delta t \sum_{j} \sum_{n}\left(D_{-} u_{j}^{n}\right)^{2}\right)^{1 / 2}+\|k\|_{\infty} \Delta x^{2} \Delta t \sum_{j} \sum_{n}\left(D_{-} u_{j}^{n}\right)^{2}\right] \mapsto 0 \text { as } \Delta x \downarrow 0 .
$$

Similarly,

$$
\begin{aligned}
\left|\mathcal{E}_{\Delta x}^{8}\right| \leq \gamma C \frac{\mu(\Delta x)}{\Delta x^{2}}\|\psi\|_{\infty}\left[|k|_{B V}\left(\Delta x^{2} \mu(\Delta x) \Delta t \sum_{j} \sum_{n}\left(D_{+}^{t} D_{-} u_{j}^{n}\right)^{2}\right)^{1 / 2}\right. & \\
\left.+\|k\|_{\infty} \Delta x^{2} \mu(\Delta x) \Delta t \sum_{j} \sum_{n}\left(D_{+}^{t} D_{-} u_{j}^{n}\right)^{2}\right] & \mapsto 0 \text { as } \Delta x \downarrow 0 .
\end{aligned}
$$


Finally, we are left with the term $\mathcal{Q}_{\Delta x}^{4}$. We see that

$$
\begin{aligned}
\mathcal{Q}_{\Delta x}^{4}:= & \frac{1}{2} \Delta x \sum_{j} \sum_{n} \psi_{j}^{n} \eta^{\prime \prime}\left(\theta_{1}\right)\left(u_{j}^{n+1}-w_{j}^{n}\right)^{2} \leq \underbrace{\beta^{2} \Delta x \sum_{j} \sum_{n} \psi_{j}^{n} \eta^{\prime \prime}\left(\theta_{1}\right)\left(\Delta x \Delta t D_{+} D_{-} u_{j}^{n}\right)^{2}}_{\mathcal{E}_{\Delta x}^{9}} \\
& +\underbrace{\gamma^{2} \Delta x \sum_{j} \sum_{n} \psi_{j}^{n} \eta^{\prime \prime}\left(\theta_{1}\right)\left(\mu(\Delta x) \Delta t D_{t}^{+} D_{+} D_{-} u_{j}^{n}\right)^{2}}_{\mathcal{E}_{\Delta x}^{10}} .
\end{aligned}
$$

We start with the first term $\mathcal{E}_{\Delta x}^{9}$. Making use of the a priori estimate (4.3b), we conclude

$$
\left|\mathcal{E}_{\Delta x}^{9}\right| \leq C \frac{\mu(\Delta x)}{\Delta x^{2}}\left(\Delta x^{2} \Delta t \sum_{j} \sum_{n}\left(D_{-} u_{j}^{n}\right)^{2}\right)\|\psi\|_{\infty} \mapsto 0 \text { as } \Delta x \downarrow 0 .
$$

Similarly, making use of the a priori estimate (4.3d), we argue that

$$
\left|\mathcal{E}_{\Delta x}^{10}\right| \leq C \frac{\mu(\Delta x)^{2}}{\Delta x^{4}}\left(\Delta x^{2} \mu(\Delta x) \Delta t \sum_{j} \sum_{n}\left(D_{+}^{t} D_{-} u_{j}^{n}\right)^{2}\right)\|\psi\|_{\infty} \mapsto 0 \text { as } \Delta x \downarrow 0 .
$$

Now in view of the above result, we are ready to prove the following lemma:

Lemma 6.2. Let $u(x, t)$ be a weak solution constructed as the limit of the approximation $u_{\Delta x}$ generated by the scheme (3.2) with $\mu(\Delta x)=\mathcal{O}\left(\Delta x^{2}\right)$. For the test function $\psi \geq 0$ with compact support in $t>0, x \in \mathbb{R} \backslash \Omega$, and every $c \in \mathbb{R}$, the following entropy inequality holds

$$
\begin{aligned}
\int_{\mathbb{R}} \int_{0}^{T}\left(|u-c| \psi_{t}+\operatorname{sign}(u-c) k(x)(f(u)-f(c)) \psi_{x}\right) \mathrm{d} x \mathrm{~d} t & \\
& +\int_{\mathbb{R}}\left|u_{0}-c\right| \psi(x, 0) \mathrm{d} x-\int_{\mathbb{R} \backslash \Omega} \int_{0}^{T} \operatorname{sign}(u-c) k^{\prime}(x) f(c) \psi \mathrm{d} x \mathrm{~d} t \geq 0 .
\end{aligned}
$$

Proof. A simple manifestation of the above Lemma 6.1 for the specific entropy $\eta(u)=|u-c|$ and consequently the entropy flux function $Q(u)=\operatorname{sign}(u-c)(f(u)-f(c))$ essentially completes the proof.

Lemma 6.3. Let $u(x, t)$ be a weak solution constructed as the limit of the approximation $u_{\Delta x}$ generated by the scheme (3.2) with $\mu(\Delta x)=\mathcal{O}\left(\Delta x^{2}\right)$. Let $0 \leq \psi \in \mathcal{D}(\mathbb{R} \times[0, T])$. Then the following entropy inequality is satisfied for all $c \in \mathbb{R}$

$$
\begin{aligned}
& \int_{\mathbb{R}} \int_{0}^{T}\left(|u-c| \psi_{t}\right.\left.+\operatorname{sign}(u-c) k(x)(f(u)-f(c)) \psi_{x}\right) \mathrm{d} x \mathrm{~d} t+\int_{\mathbb{R}}\left|u_{0}-c\right| \psi(x, 0) \mathrm{d} x \\
&+|f(c)| \int_{\mathbb{R} \backslash \Omega} \int_{0}^{T}\left|k^{\prime}(x)\right| \psi \mathrm{d} x \mathrm{~d} t+\sum_{m=1}^{M} \int_{0}^{T}\left|f(c)\left(k_{m}^{+}-k_{m}^{-}\right)\right| \psi\left(\xi_{m}, t\right) \mathrm{d} t \geq 0 .
\end{aligned}
$$

Proof. A straightforward adaptation of ([14], Lem. 4.2) along with the help of Lemma 6.1 concludes the proof. 
To proceed further, we combine above two lemma's. In what follows, we have the following important theorem:

Theorem 6.4. Let $u(x, t)$ be a weak solution constructed as the limit of the approximation $u_{\Delta x}$ generated by the scheme (3.2) with $\mu(\Delta x)=\mathcal{O}\left(\Delta x^{2}\right)$. Let $0 \leq \psi \in \mathcal{D}(\mathbb{R} \times[0, T])$. Then the following entropy inequality is satisfied for all $c \in \mathbb{R}$

$$
\begin{aligned}
\int_{\mathbb{R}} \int_{0}^{T}(|u-c| & \left.\psi_{t}+\operatorname{sign}(u-c) k(x)(f(u)-f(c)) \psi_{x}\right) \mathrm{d} x \mathrm{~d} t+\int_{\mathbb{R}}\left|u_{0}-c\right| \psi(x, 0) \mathrm{d} x \\
& +\int_{\mathbb{R} \backslash \Omega} \int_{0}^{T} \operatorname{sign}(u-c) k^{\prime}(x) f(c) \psi \mathrm{d} x \mathrm{~d} t+\sum_{m=1}^{M} \int_{0}^{T}\left|f(c)\left(k_{m}^{+}-k_{m}^{-}\right)\right| \psi\left(\xi_{m}, t\right) \mathrm{d} t \geq 0 .
\end{aligned}
$$

Proof. A verbatim copy of the proof of ([14], Lem. 4.4) ensures the proof.

\subsection{Uniqueness of entropy solutions}

Following [14], we mention that entropy solutions are unique under a crossing condition. It is well-known that one has to impose the crossing condition only because the entropy inequality (1.2) alone is not sufficient to guarantee uniqueness when the crossing condition is violated. However, we mention that in the multiplicative case $f(k, u)=k(x) f(u)$ there is no flux crossing, hence we don't assume any such crossing condition.

One more technical issue is the existence of traces along the discontinuity curves $\omega_{m}$, for $m=1,2, \ldots, M$. Without going into the details of it, we simply mention one instance where we automatically have the existence of strong traces. We remark that, due to the genuinely nonlinearity assumption, existence of traces is guaranteed when $k(x)$ is constant on each region $\mathcal{R}_{m}$, for $m=1,2, \ldots, M$. This is a consequence of a general result by Vasseur [31]. For more general case, we encourage the readers to consult [14] for general assumptions ensuring the existence of traces.

To this end, we collect all the above mentioned results in the following theorem.

Theorem 6.5. Assume that assumptions A.1, A.2, A.3, A.4, and 4 hold. Moreover, suppose that the addition regularity assumptions on the discontinuous coefficient $k$ holds, and the traces along the discontinuity curves exist. Then a unique entropy solution to the Cauchy problem (1.4) exists and the entire computed sequence of approximations $\left\{u_{\Delta x}\right\}_{\Delta x>0}$, generated by the scheme (3.2) with $\mu(\Delta x)=\mathcal{O}\left(\Delta x^{2}\right)$, converges to the unique entropy solution of (1.4).

\section{Diffusive-DisPersive APPROXIMATIONS}

As we already pointed out in the introduction, all the aforementioned techniques can be utilized to analyze the equation given by the diffusive dispersive approximations of scalar conservation laws with a discontinuous flux of the type

$$
\begin{cases}u_{t}^{\varepsilon}+f\left(k(x), u^{\varepsilon}\right)_{x}=\varepsilon \beta u_{x x}^{\varepsilon}+\mu(\varepsilon) \gamma u_{x x x}^{\varepsilon}, & x \in \mathbb{R} \times(0, T), \\ u^{\varepsilon}(x, 0)=u_{0}(x), & x \in \mathbb{R},\end{cases}
$$

when $\varepsilon>0$ tends to zero with $0<\mu(\varepsilon) \mapsto 0$ as $\varepsilon \mapsto 0$. Here $T>0$ is fixed, $\beta, \gamma>0$ are fixed parameters, $u^{\varepsilon}: \mathbb{R} \times[0, T) \mapsto \mathbb{R}$ is the unknown scalar map, $u_{0}$ the initial data, $k: \mathbb{R} \mapsto \mathbb{R}$ is a spatially varying coefficient, and the flux function $f: \mathbb{R}^{2} \mapsto \mathbb{R}$ is a sufficiently smooth scalar function.

We propose the following fully-discrete (in space and time) finite difference scheme approximating the limiting solutions generated by equation (7.1)

$$
\begin{aligned}
D_{+}^{t} u_{j}^{n}+D_{-} h_{j+\frac{1}{2}}^{n} & =\beta \Delta x D_{+} D_{-} u_{j}^{n}+\gamma \mu(\Delta x) D_{+} D_{-}^{2} u_{j}^{n}, \quad j \in \mathbb{Z}, n \in \mathbb{N}_{0}, \\
u_{j}^{0} & =\frac{1}{\Delta x} \int_{x_{j-\frac{1}{2}}}^{x_{j+\frac{1}{2}}} u_{0}(\theta) \mathrm{d} \theta, \quad j \in \mathbb{Z},
\end{aligned}
$$


where $\beta, \gamma>0$ are fixed parameters, and $\mu(\Delta x) \mapsto 0$ as $\Delta x \mapsto 0$. As before, we will either use $\mu(\Delta x)=\mathcal{O}\left(\Delta x^{2}\right)$ or $\mu(\Delta x)=\mathcal{O}\left(\Delta x^{2}\right)$ depending on the quest for the convergence of approximate solution $u_{\Delta x}$ towards a weak solution or the entropy solution, respectively. Note that in this case, in contrast to the a priori estimates in Section 4 , we have the following estimates

Lemma 7.1. Let $u_{\Delta x}$ be a sequence of approximations generated by the scheme (7.2)-(7.3). Moreover, assume that the initial data $u_{0}$ lies in $L^{2}(\mathbb{R})$. Then the following estimate holds

$$
\frac{1}{2} D_{+}^{t}\left\|u^{n}\right\|^{2}+\delta \frac{\gamma \Delta x \mu(\Delta x)}{2}\left\|D_{-}^{2} u^{n}\right\|^{2}+\delta \Delta x\left\|D_{-} u^{n}\right\|^{2} \leq C,
$$

provided $\Delta t$ and $\Delta x$ satisfies the following CFL condition

$$
\max \left\{2 \lambda\left(\beta^{2} \frac{\Delta x^{2}}{\gamma \mu(\Delta x)}+2 \frac{\gamma \mu(\Delta x)}{\Delta x^{2}}\right), 8 \lambda\|k\|^{2}\left\|f^{\prime}\right\|^{2}\right\} \leq \min (1-\delta, \beta-\delta), \quad \delta \in(0, \min (1, \beta)),
$$

where $\lambda=\Delta t / \Delta x$ and the constant $C>0$ is independent of $\Delta x$.

In particular, the estimate (7.4) guarantees following space-time estimates:

$$
\begin{array}{r}
\forall n \in \mathbb{N}, \quad \Delta x \sum_{j}\left(u_{j}^{n}\right)^{2} \leq C, \\
\Delta x^{2} \Delta t \sum_{j} \sum_{n}\left(D_{-} u_{j}^{n}\right)^{2} \leq C, \\
\Delta t \Delta x^{2} \mu(\Delta x) \sum_{j} \sum_{n}\left(D_{-}^{2} u_{j}^{n}\right)^{2} \leq C . \\
\Delta x^{2} \Delta t \sum_{j} \sum_{n}\left(D_{+}^{t} u_{j}^{n}\right)^{2} \leq C .
\end{array}
$$

Proof. To start with, we multiply the scheme (7.2) by $\Delta x u_{j}^{n}$ and subsequently sum over $j \in \mathbb{Z}$. Then, using summation-by-parts formula and the identity (3.1), we obtain

$$
\begin{aligned}
& \frac{1}{2} D_{+}^{t} \sum_{j} \Delta x\left(u_{j}^{n}\right)^{2}-\frac{\Delta t}{2} \sum_{j} \Delta x\left(D_{+}^{t} u_{j}^{n}\right)^{2}+\underbrace{\Delta x \sum_{j} u_{j}^{n} D_{-} h_{j+\frac{1}{2}}^{n}}_{\mathcal{I}_{\Delta x}(f)} \\
&=-\beta \Delta x \sum_{j} \Delta x\left|D_{-} u_{j}\right|^{2}-\gamma \mu(\Delta x) \Delta x \sum_{j} D_{-} u_{j}^{n} D_{-}\left(D_{-} u_{j}^{n}\right) .
\end{aligned}
$$

Using the identity

$$
u_{j}^{n} D_{-} u_{j}^{n}=\frac{1}{2} D_{-}\left(u_{j}^{n}\right)^{2}+\frac{\Delta x}{2}\left(D_{-} u_{j}^{n}\right)^{2}
$$

which is very similar to $(3.1)$, we get

$$
\gamma \mu(\Delta x) \Delta x \sum_{j} D_{-} u_{j}^{n} D_{-}\left(D_{-} u_{j}^{n}\right)=\underbrace{\gamma \frac{\Delta x \mu(\Delta x)}{2} \sum_{j} D_{-}\left(D_{-} u_{j}^{n}\right)^{2}}_{=0}+\frac{\gamma \Delta x^{2} \mu(\Delta x)}{2} \sum_{j}\left(D_{-}^{2} u_{j}^{n}\right)^{2} .
$$

Furthermore, it has already been shown in the proof of Lemma 4.2 that

$$
-\mathcal{I}_{\Delta x}(f) \leq C
$$


where $C$ is independent of $\Delta x$. Thus, we have the estimate

$$
\frac{1}{2} D_{+}^{t}\left\|u^{n}\right\|^{2} \leq \frac{\Delta t}{2}\left\|D_{+}^{t} u^{n}\right\|^{2}-\beta \Delta x\left\|D_{-} u^{n}\right\|^{2}-\frac{\gamma \Delta x \mu(\Delta x)}{2}\left\|D_{-}^{2} u^{n}\right\|^{2}+C .
$$

Noting that $\Delta x D_{+} D_{-}^{2}=D_{-}\left(D_{+}-D_{-}\right)$, we use the scheme (7.2) to get the relation

$$
\begin{aligned}
\frac{\Delta t}{2}\left\|D_{+}^{t} u^{n}\right\|^{2} \leq \Delta t\left\|D_{-} h_{j+\frac{1}{2}}^{n}\right\|^{2} & +\beta^{2} \Delta t \Delta x^{2}\left\|D_{+} D_{-} u^{n}\right\|^{2} \\
& +\frac{\gamma^{2} \Delta t \mu(\Delta x)^{2}}{\Delta x^{2}}\left\|D_{-} D_{+} u^{n}\right\|^{2}+\frac{\gamma^{2} \Delta t \mu(\Delta x)^{2}}{\Delta x^{2}}\left\|D_{-}^{2} u^{n}\right\|^{2} .
\end{aligned}
$$

Now

$$
\begin{aligned}
\Delta t\left\|D_{-} h_{j+\frac{1}{2}}^{n}\right\|^{2} & =\lambda \sum_{j}\left(k_{j+\frac{1}{2}} \hat{f}_{j+\frac{1}{2}}^{n}-k_{j-\frac{1}{2}} \hat{f}_{j-\frac{1}{2}}^{n}\right)^{2} \\
& =\lambda \sum_{j}\left(k_{j+\frac{1}{2}} \hat{f}_{j+\frac{1}{2}}^{n}-k_{j-\frac{1}{2}} \hat{f}_{j+\frac{1}{2}}^{n}+k_{j-\frac{1}{2}} \hat{f}_{j+\frac{1}{2}}^{n}-k_{j-\frac{1}{2}} \hat{f}_{j-\frac{1}{2}}^{n}\right)^{2} \\
& \leq 2 \lambda \sum_{j}\left(k_{j+\frac{1}{2}}-k_{j-\frac{1}{2}}\right)^{2}\left(\hat{f}_{j+\frac{1}{2}}^{n}\right)^{2}+\left(k_{j-\frac{1}{2}}\right)^{2}\left(\hat{f}_{j+\frac{1}{2}}^{n}-\hat{f}_{j-\frac{1}{2}}^{n}\right)^{2} .
\end{aligned}
$$

Since EO flux is Lipschitz continuous with Lipschitz constant $\left\|f^{\prime}\right\|_{\infty}(c f .(3.5))$, we conclude that

$$
\operatorname{supsup}_{j}\left|\hat{f}_{j+\frac{1}{2}}^{n}\right| \leq\|f\|_{\infty}, \text { and }\left|\hat{f}_{j+\frac{1}{2}}^{n}-\hat{f}_{j-\frac{1}{2}}^{n}\right| \leq\left\|f^{\prime}\right\|_{\infty}\left(\left|u_{j}^{n}-u_{j-1}^{n}\right|+\left|u_{j+1}^{n}-u_{j}^{n}\right|\right) .
$$

Thus, we get

$$
\Delta t\left\|D_{-} h_{j+\frac{1}{2}}^{n}\right\|^{2} \leq 2 \lambda\|k\|\|f\|^{2}|k|_{B V}+4 \Delta t\|k\|^{2}\left\|f^{\prime}\right\|^{2}\left(\left\|D_{-} u^{n}\right\|^{2}+\left\|D_{+} u^{n}\right\|^{2}\right) .
$$

Using relations (7.8),(7.9) and (7.10) in tandem with the fact that $\left\|D_{-}(.)^{n}\right\|=\left\|D_{+}(.)^{n}\right\|$, we get the estimate

$$
\frac{1}{2} D_{+}^{t}\left\|u^{n}\right\|^{2}+\left(1-2 \frac{\beta^{2} \lambda \Delta x^{2}}{\gamma \mu(\Delta x)}-4 \frac{\gamma \lambda \mu(\Delta x)}{\Delta x^{2}}\right) \frac{\gamma \Delta x \mu(\Delta x)}{2}\left\|D_{-}^{2} u^{n}\right\|^{2}+\left(\beta-8 \lambda\|k\|^{2}\left\|f^{\prime}\right\|^{2}\right) \Delta x\left\|D_{-} u^{n}\right\|^{2} \leq C
$$

Choosing $\lambda$ in accordance to (7.5) ensures that for some $\delta \in(0, \min (\beta, 1))$

$$
1-2 \frac{\beta^{2} \lambda \Delta x^{2}}{\gamma \mu(\Delta x)}-4 \frac{\gamma \lambda \mu(\Delta x)}{\Delta x^{2}}>\delta, \text { and } \beta-8 \lambda\|k\|^{2}\left\|f^{\prime}\right\|^{2}>\delta
$$

thus leading to the estimate (7.4).

In order to prove estimates (7.6a)-(7.6c), we multiply the inequality (7.4) by $\Delta t$ and subsequently sum over all $n=0,1, \ldots, N-1$ to reach

$$
\frac{1}{2}\left\|u^{N}\right\|^{2}+\delta \frac{\gamma \Delta x \Delta t \mu(\Delta x)}{2} \sum_{n}\left\|D_{-}^{2} u^{n}\right\|^{2}+\delta \Delta x \Delta t \sum_{n}\left\|D_{-} u^{n}\right\|^{2} \leq \frac{1}{2}\left\|u_{0}\right\|^{2}+C,
$$

This essentially finishes the proof of the a priori bounds (7.6a)-(7.6c). Using these estimates and the relation (7.9), a direct computation shows that (7.6d) holds. This completes the proof.

Note that, making use of these a priori estimates, an appropriate "convergence theorem" can be formulated, which guarantees the convergence of approximate solutions $\left\{u_{\Delta x}\right\}_{\Delta x>0}$, generated by the scheme (7.2)-(7.3), to a weak solution of (1.4). 
Theorem 7.2. Let $u_{\Delta x}$ be a sequence of approximations generated via the scheme (7.2)-(7.3) with $\mu(\Delta x)=$ $\mathcal{O}\left(\Delta x^{2}\right)$. Then there exists a function $u \in L^{\infty}\left([0, T] ; L_{\text {loc }}^{1}(\mathbb{R})\right)$ and a sequence $\left\{\Delta x_{j}\right\}$ of $\{\Delta x\}$ such that $u_{\Delta x_{j}} \mapsto u$ as $\Delta x_{j} \downarrow$ 0. Moreover, the function $u$ is a weak solution to (1.4).

Proof. The proof of this theorem is very much similar to the proof of the Theorem 5.8, except the analysis of the terms involving $D_{+} D_{-}^{2} u_{j}^{n}$. However, these term can be treated like the term $D_{+}^{t} D_{+} D_{-} u_{j}^{n}$ in Section 5 , but we need to use the a priori bound (7.6c) instead of (4.3d). For brevity of exposition, we omit the details of the proof.

A similar result, in view of the analysis in Section 6 and above a priori estimates, can be obtained regarding the convergence of $\left\{u_{\Delta x}\right\}_{\Delta x>0}$, generated by the scheme (7.2)-(7.3), to the unique entropy solution of (1.4).

Theorem 7.3. Let $u(x, t)$ be a weak solution constructed as the limit of the approximation $u_{\Delta x}$ generated by the scheme (7.2)-(7.3) with $\mu(\Delta x)=\mathcal{O}\left(\Delta x^{2}\right)$. Let $0 \leq \psi \in \mathcal{D}(\mathbb{R} \times[0, T])$. Then the following entropy inequality is satisfied for all $c \in \mathbb{R}$

$$
\begin{aligned}
\int_{\mathbb{R}} \int_{0}^{T}\left(|u-c| \psi_{t}+\operatorname{sign}(u-c) k(x)(f(u)-f(c)) \psi_{x}\right) \mathrm{d} x \mathrm{~d} t+\int_{\mathbb{R}}\left|u_{0}-c\right| \psi(x, 0) \mathrm{d} x \\
\quad+\int_{\mathbb{R} \backslash \Omega} \int_{0}^{T} \operatorname{sign}(u-c) k^{\prime}(x) f(c) \psi \mathrm{d} x \mathrm{~d} t+\sum_{m=1}^{M} \int_{0}^{T}\left|f(c)\left(k_{m}^{+}-k_{m}^{-}\right)\right| \psi\left(\xi_{m}, t\right) \mathrm{d} t \geq 0 .
\end{aligned}
$$

Proof. This can be achieved using similar arguments used in the proof of Theorem 6.4.

\section{NumERICAL EXPERIMENTS}

We present a few numerical results to substantiate the results we have shown in the previous sections. We consider the capillarity problem approximated by the scheme (3.2), as well as the diffusive-dispersive problem approximated by (7.2). Note that while the former is approximated by an implicit type scheme, the latter is an explicit-in-time scheme.

\subsection{Capillarity approximation}

We consider the flow of two phases in a heterogeneous porous medium, in the limit of vanishing dynamic capillary pressure. The model equation is given by

$$
u_{t}+f(k(x), u)_{x}=\varepsilon \beta\left(g(k(x), u) u_{x}\right)_{x}+\mu(\varepsilon) \gamma\left(h(k(x), u) u_{x t}\right)_{x}, \quad x \in \mathbb{R} \times(0, T),
$$

where $g, h: \mathbb{R}^{2} \rightarrow \mathbb{R}$ are assumed to be smooth functions such that

$$
\alpha \leq g(., .), h(., .)
$$

for some constant $\alpha>0$. This model has also been considered and numerically analysed in $[4,16,30]$. Note that, we have theoretically shown the convergence for the special case when $g \equiv h \equiv 1$. However, we mention that this cosmetic changes in the equation has no effect on the central idea of the paper and a straightforwardly incremental modification of our analysis can be adopted to analyze equation (8.1). We have decide to work with this equation because of the availability of results for such equations which help us to compare our results. 
As done in [4], we choose the various quantititis in the model as follows:

$$
\begin{aligned}
f(k(x), u) & =\frac{z^{w}\left(1-k(x) z^{o}\right)}{z^{w}+z^{o}}, \\
g(k(x), u) & =k(x) g_{1}(u)=k(x) \frac{z^{w} z^{o}}{z^{w}+z^{o}} P^{\prime}(u), \\
h(k(x), u) & =k(x) h_{1}(u)=k(x) \frac{z^{w} z^{o}}{z^{w}+z^{o}}, \\
P(u) & =\left(u^{-\frac{4}{3}}-1\right)^{\frac{1}{4}},
\end{aligned}
$$

where $z^{w}=u^{2}$ and $z^{o}=(1-u)^{2}$. From the physical point of view, $u(x, t)$ and $(1-u(x, t))$ represent the water and oil saturations respectively, while $k(x)$ corresponds to the rock permeability. The corresponding modified numerical approximation is chosen to be

$$
\begin{aligned}
D_{+}^{t} u_{j}^{n}+D_{-} h_{j+\frac{1}{2}}^{n}= & \beta \Delta x D_{+}\left(k_{j-\frac{1}{2}} \frac{\left(g_{1}\left(u_{j}^{n}\right)+g_{1}\left(u_{j-1}^{n}\right)\right)}{2} D_{-} u_{j}^{n}\right) \\
& +\gamma \mu(\Delta x) D_{+}\left(k_{j-\frac{1}{2}} \frac{\left(h_{1}\left(u_{j}^{n}\right)+h_{1}\left(u_{j-1}^{n}\right)\right)}{2} D_{+}^{t} D_{-} u_{j}^{n}\right) .
\end{aligned}
$$

As stated earlier, we can replace the EO numerical flux with any other monotone flux, with a similar covergence analysis following through. For simplicity, we choose the Lax Friedrichs flux for the present model. The spatial domain is $[0,2]$ with an initial solution profile

$$
u_{0}(x)= \begin{cases}0.8 & \text { for } x \leq 0.25 \\ 0.2 & \text { for } x>0.25\end{cases}
$$

Furthermore, we choose $\beta=6, \gamma=36$ and CFL 0.3, with the final time of simulation being $T=0.6$.

\subsubsection{Continuous flux}

We first consider the scenario when $k \equiv 1$. This corresponds to the flow in a homogeneous rock structure. When the dispersion coefficient is chosen as $\mu(\Delta x)=\Delta x^{2}$, the numerical solution converges to a weak solution of (8.1), as shown in Figure 1. The weak solution consists of a leading classical shock wave and a trailing nonclassical shock wave, with an intermediate state in between. However, when the dispersion coefficient is chosen to $\mu(\Delta x)=\Delta x^{3}$, the solution converges to the entropy solution, as shown in Figure 2. This is in accordance with Theorem 6.5.

\subsubsection{Discontinuous flux}

We next consider the scenario depicting the flow through a heterogeneous medium. We chose the rock permeability as

$$
k(x)= \begin{cases}1.1 & \text { for } x \leq 0.6 \\ 1.4 & \text { for } x>0.6\end{cases}
$$

which corresponds to two rock types with a sharp interface at $x=0.6$. As before we first consider the solution by setting $\mu(\Delta x)=\Delta x^{2}$, which is shown in Figure 3. The numerical approximation is a weak solution consisting of a leading classical shock wave and a trailing non-classical shock wave separated by an intermediate state, and discontinuity at $x=0.6$ corresponding to rock structure. Once again,the non-classical shock disappears when the dispersion coefficient is chosen as $\mu(\Delta x)=\Delta x^{3}$, with the solution approximating the entropy solution. 


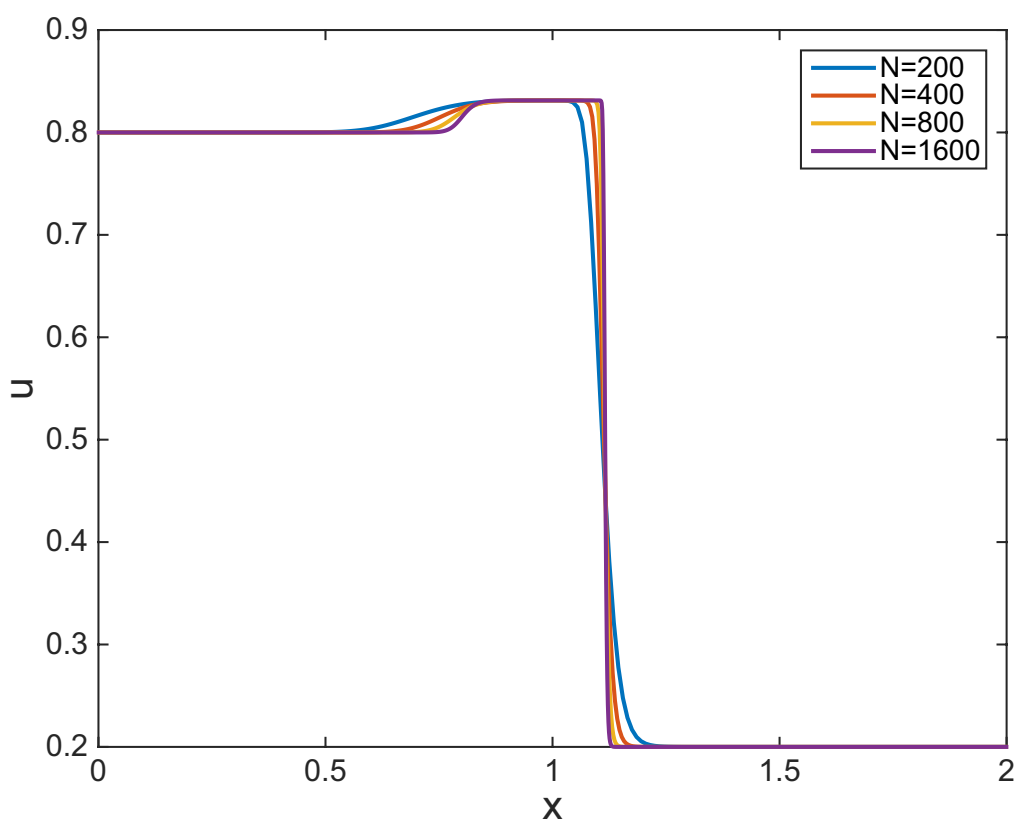

Figure 1. Two phase flow through a homogeous medium $(k(x) \equiv 1)$ at time $T=0.6$ with $\mu(\Delta x)=\Delta x^{2}$. Mesh refinement study indicates convergence to a non-classical weak solution of the underlying conservation law.

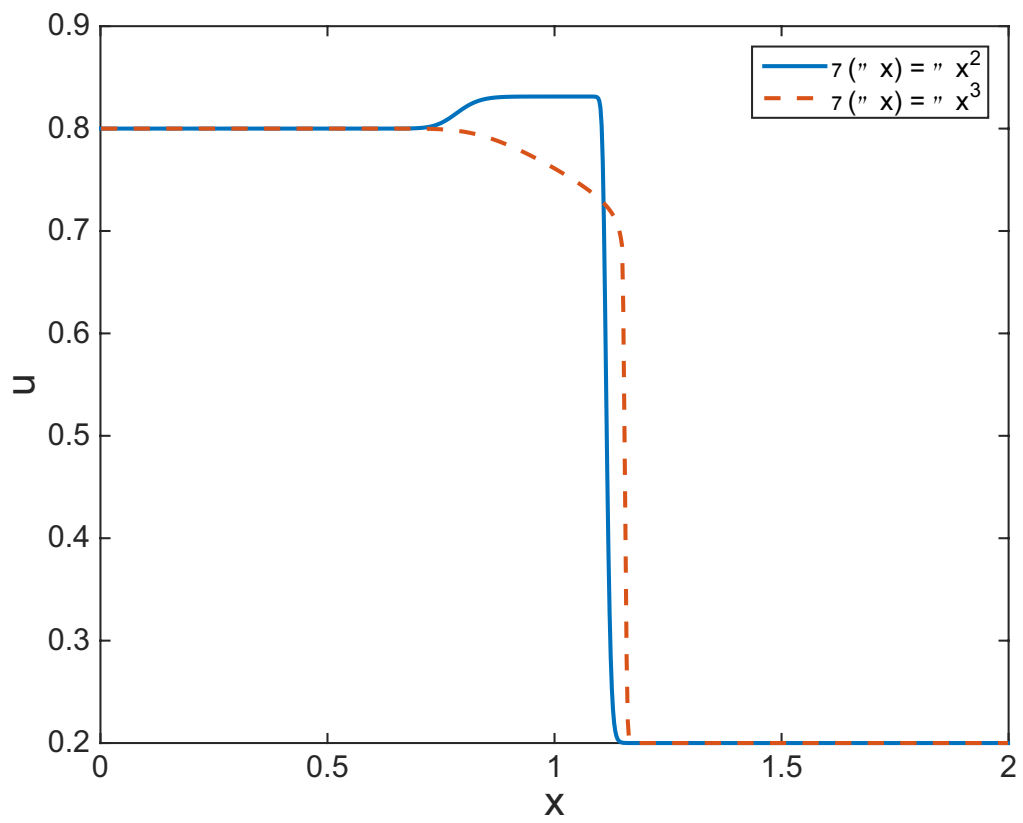

Figure 2. Two phase flow through a homogeous medium $(k(x) \equiv 1)$ at time $T=0.6$. A non-classical weak solution is obtained for $\mu(\Delta x)=\Delta x^{2}$, while the unique entropy solution is obtained for $\mu(\Delta x)=\Delta x^{3}$. 


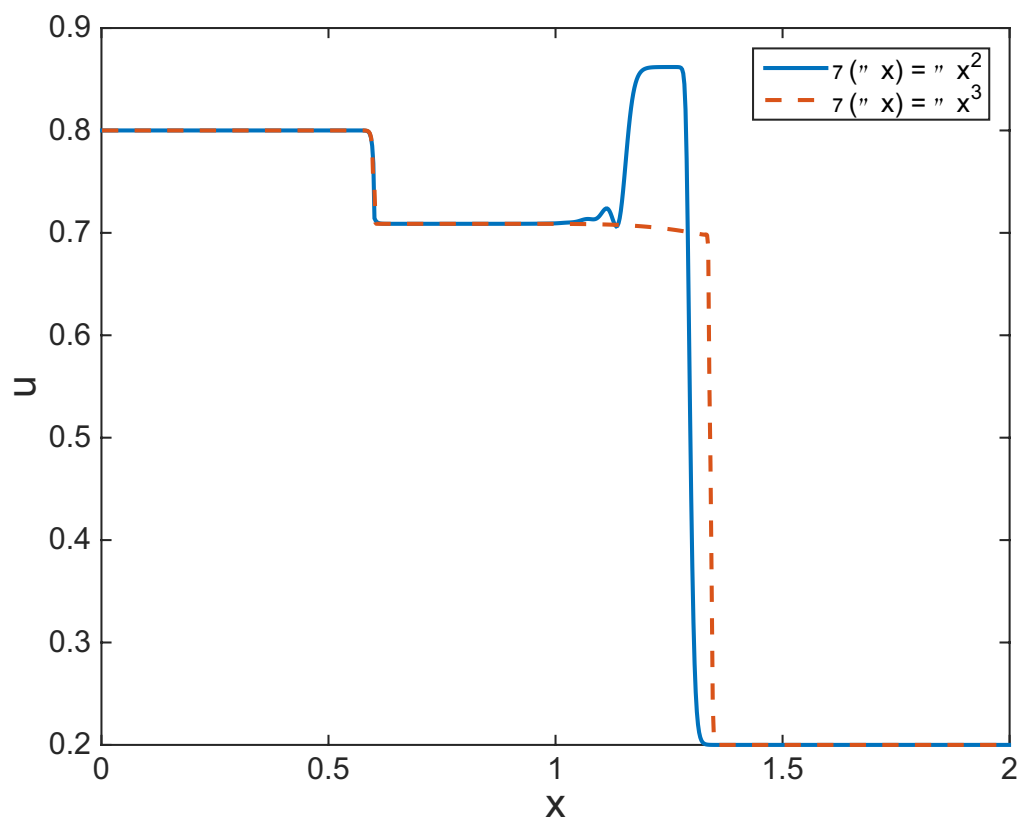

Figure 3. Two phase flow through a hetergeneous medium at time $T=0.6$. A non-classical weak solution is obtained for $\mu(\Delta x)=\Delta x^{2}$, while the unique entropy solution is obtained for $\mu(\Delta x)=\Delta x^{3}$.

\subsection{Diffusive-dispersive model}

As we have already mentioned, the convergence analysis for the diffusive-dispersive equation (7.1) is very similar to the other. We work with the flux function

$$
f(k(x), u)=k(x)\left(u^{3}-u\right)
$$

and the numerical scheme given by (7.3). The continuous flux version of this problem has been studied numerically in $[2]$ as well. The spatial domain is $[-0.5,0.5]$ with the final simulation time being $T=0.01$. The initial profile of the solution for this problem is taken to be

$$
u_{0}(x)=\left\{\begin{array}{lr}
4 & \text { for } x \leq 0 \\
-2 & \text { for } x>0
\end{array}\right.
$$

with the model parameters chosen as $\beta=5$ and $\gamma=20$. For this problem, the EO numerical flux is used.

\subsubsection{Continuous flux}

We first work with a continuous flux by choosing $k(x) \equiv 1$. For $\mu(\Delta x)=\Delta x^{2}$, the numerical solution converges to a weak solution (see Fig. 4) consisting of trailing classical shock wave and a leading non-classical shock wave separated by an intermediate state. Figure 5 shows that for the choice $\mu(\Delta x)=\Delta x^{2.5}$, we get an approximation to the unique entropy solution, which corresponds to a single shock wave satisfying Olënik's entropy condition [25]. 


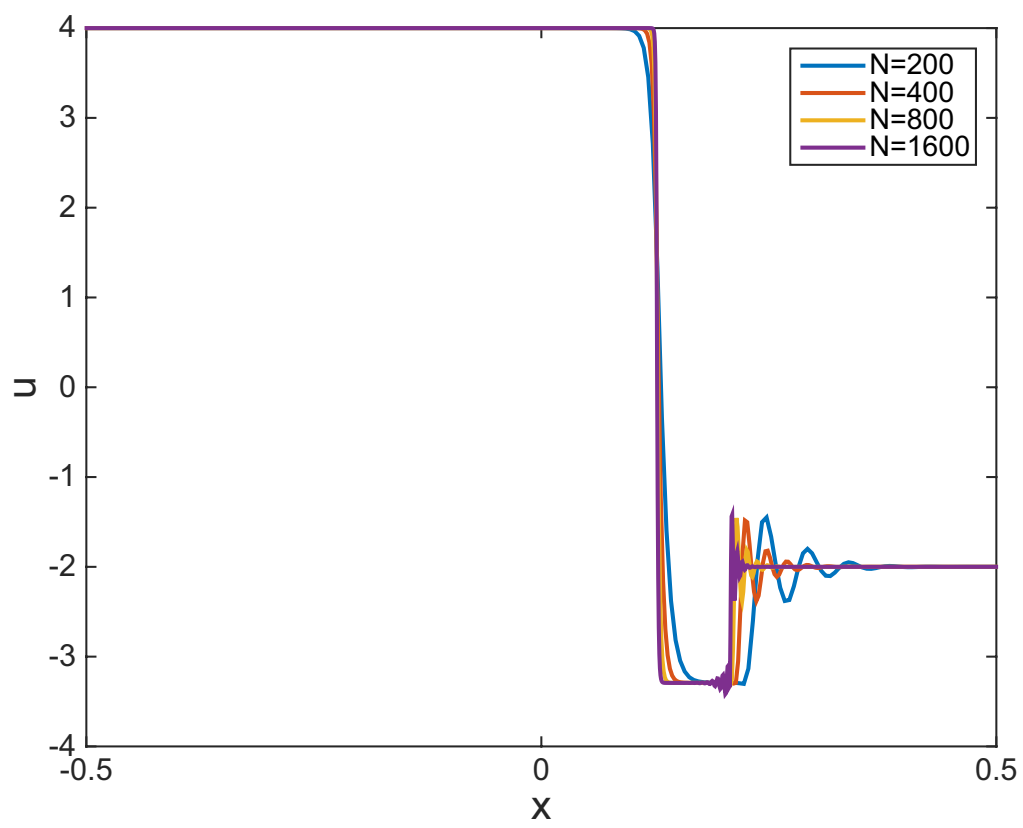

Figure 4. Diffusive-dispersive model with $k(x) \equiv 1$, at time $T=0.01$ and $\mu(\Delta x)=\Delta x^{2}$. Mesh refinement study indicates convergence to a non-classical weak solution of the underlying conservation law.

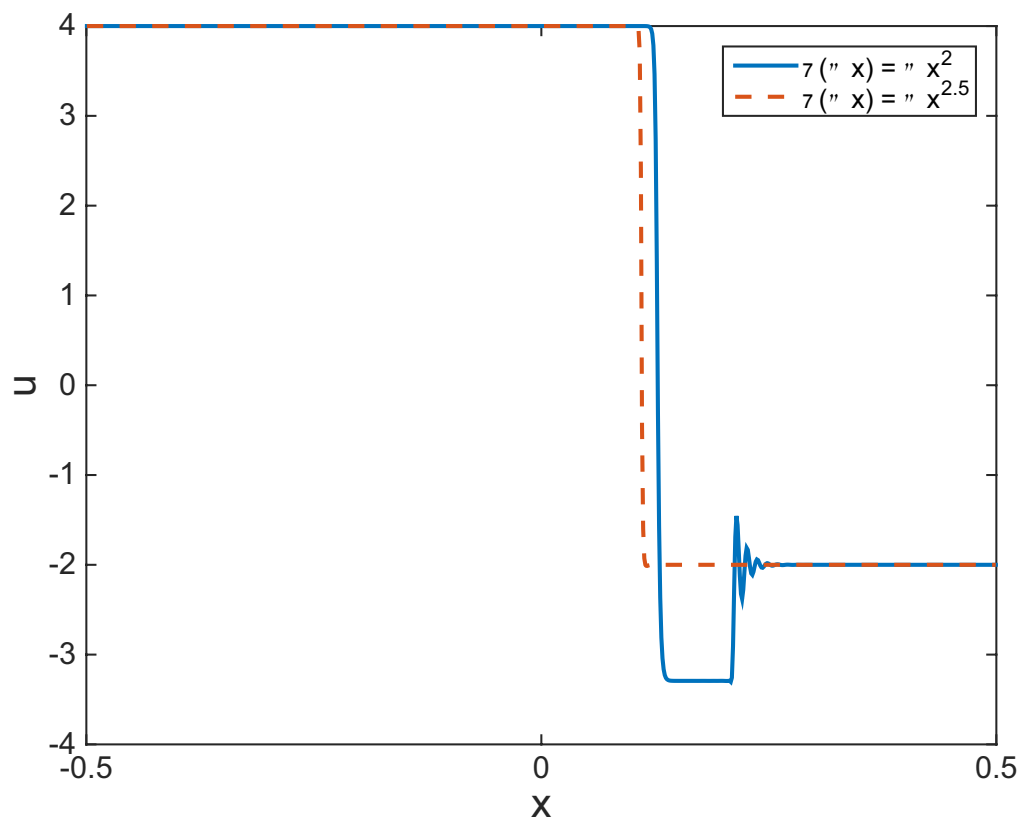

FiguRE 5. Diffusive-dispersive model with $k(x) \equiv 1$, at time $T=0.01$. A non-classical weak solution is obtained for $\mu(\Delta x)=\Delta x^{2}$, while the unique entropy solution is obtained for $\mu(\Delta x)=\Delta x^{3}$. 


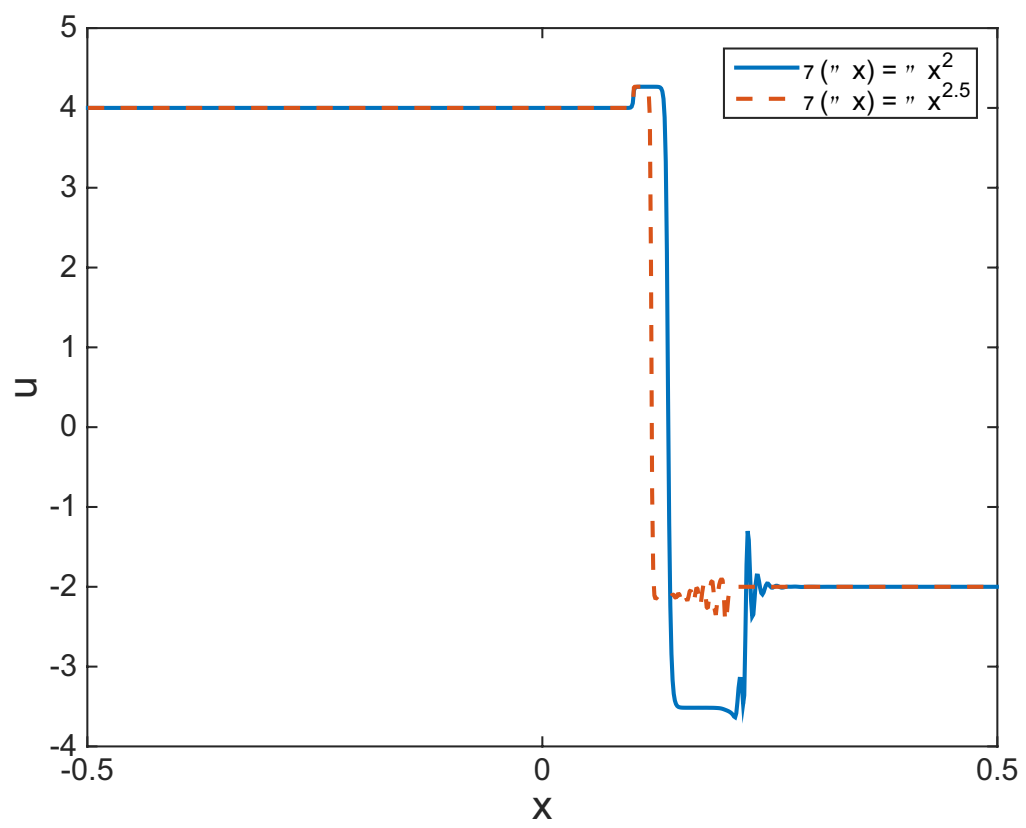

Figure 6. Diffusive-dispersive model with a discontinuous flux, at time $T=0.01$. A nonclassical weak solution is obtained for $\mu(\Delta x)=\Delta x^{2}$, while the unique entropy solution is obtained for $\mu(\Delta x)=\Delta x^{3}$.

\subsubsection{Discontinuous flux}

We work with a discontinuous flux characterised by

$$
k(x)= \begin{cases}1.1 & \text { for } x \leq 0.1 \\ 0.9 & \text { for } x>0.1\end{cases}
$$

The numerical approximation shown in Figure 6 with $\mu(\Delta x)=\Delta x^{2}$, is a weak solution consisting of a trailing classical shock wave and a leading non-classical shock wave separated by an intermediate state. In addition, there is a standing discontinuity at $x=0.1$ corresponding to discontinuiuty in the flux. The non-classical shock disappears when the dispersion coefficient is chosen as $\mu(\Delta x)=\Delta x^{2.5}$, with the solution approximating the entropy solution.

\section{REFERENCES}

[1] K. Aziz and A. Settari, Fundamentals of petroleum reservoir simulation. Applied Science Publishers, London (1979).

[2] C. Chalons and P.G. Lefloch, A fully-discrete scheme for diffusive-dispersive conservation laws. Numer. Math. 89 (2001) $493-509$.

[3] G.-Q. Chen, Compactness methods and nonlinear hyperbolic conservation laws. In some current topics on nonlinear conservation laws. AMS, Providence, RI (2000) 33-75.

[4] G.M. Coclite, L. di Ruvo, J. Ernest and S. Mishra, Convergence of vanishing capillarity approximations for scalar conservation laws with discontinuous fluxes. Netw. Heterog. Media. 8 (2013) 969-984.

[5] R.J. DiPerna, Convergence of approximate solutions to conservation laws. Arch. Ration. Mech. Anal. 82 (1983) $27-70$.

[6] J. Ernest, P.G. Lefloch and S. Mishra, Schemes with Well-Controlled dissipation. I: Non-classical shock waves, SIAM J. Numer. Math. 53 (2015) 674-699.

[7] S.M. Hassanizadeh and W.G. Gray, Mechanics and thermodynamics of multiphase flow in porous media including interphase boundaries. Adv. Water Resour. 13 (1990) 169-186. 
[8] B.T. Hayes and P.G. Lefloch, Nonclassical shock waves and kinetic relations. Strictly hyperbolic systems, Preprint no. 357. CMAP, Ecole Polytechnique, Palaiseau, France (1996).

[9] B.T. Hayes and P.G. Lefloch, Nonclassical shocks and kinetic relations: strictly hyperbolic systems. SIAM. J. Math. Anal. 31 (2000) 941-991.

[10] T.Y. Hou and P.G. Lefloch, Why nonconservative schemes converge to wrong solutions. Error analysis. Math. Comput. 62 (1994) 497-530.

[11] S. Hwang and A.E. Tzavaras, Kinetic decomposition of approximate solutions to conservations laws: Application to relaxation and diffusion-dispersion approximations. Commun. Partial Differ. Equ. 27 (2002) 1229-1254.

[12] D. Jacobs, W.R. McKinney and M. Shearer, Traveling wave solutions of the modified Korteweg-deVries Burgers equation. J. Differ. Equ. 116 (1995) 448-467.

[13] K.H. Karlsen and J.D. Towers, Convergence of the Lax-Friedrichs scheme and stability for conservation laws with a discontinous space-time dependent flux, Chinese Ann. Math. Ser. B. 25 (2004) 287-318.

[14] K.H. Karlsen, N.H. Risebro and J.D. Towers, $L^{1}$ stability for entropy solutions of nonlinear degenerate parabolic convectiondiffusion equations with discontinuous coefficients. Skr. K. Nor. Vidensk. Selsk. 3 (2003) 1-49.

[15] K.H. Karlsen, S. Mishra and N.H. Risebro, Convergence of finite volume schemes for triangular systems of conservation laws. Numer. Math. 111 (2008) 559-589.

[16] F. Kissling and C. Rohde, The computation of nonclassical shock waves with a heterogeneous multi-scale method. Netw. Heterog. Media. 5 (2010) 661-674.

[17] F. Kissling and K.H. Karlsen, On the singular limit of a two-phase flow equation with heterogeneities and dynamic capillary pressure. ZAMM, Z. Angew. Math. Mech. 94 (2014) 678-689.

[18] C.I. Kondo and P.G. Lefloch, Zero diffusion-dispersion limits for scalar conservation laws. SIAM J. Math. Anal. 33 (2002) $1320-1329$,.

[19] S.N. Kružkov, First order quasilinear equations with several independent variables. Mat. Sb. (N.S) 81 (1970) $228-255$.

[20] P.G. Lefloch, Hyperbolic Systems of Conservation Laws: The Theory of Classical and Nonclassical Shock Waves. Lectures in Mathematics. Birkhäuser, Basel (2002).

[21] P.G. Lefloch and S. Mishra, Numerical methods with controlled dissipation for small-scale dependent shocks. Acta Numer. 23 (2014) 743-816.

[22] R.J. Leveque, Numerical Methods for Conservation Laws. Birkhauser Verlag, Boston (1992).

[23] Y. Lu, Hyperbolic conservation laws and the compensated compactness method. Vol. 128 of Chapman and Hall/CRC Monographs and surveys in Pure and Applied Mathematics. Chapman and Hall/CRC, Boca Raton, FL (2003).

[24] F. Murat, Compacite par compensation. Ann. Scuola Norm. Sup. Pisa Cl. Sci. 5 (1978) 489-507.

[25] O.A. Oleĭnik, Convergence of certain difference schemes. Soviet Math. Dokl. 2 (1961) 313-316.

[26] B. Perthame and P.E. Souganidis, A limiting case for velocity averaging. Ann. Sci. E.N.S. 31 (1998) 591-598.

[27] M.E. Schonbek, Convergence of solution to nonlinear dispersive equations. Commun. Partial Differ. Equ. 7 (1982) 959-1000.

[28] L. Tartar, Compensated compactness and applications to partial differential equations. Vol. 4 of Research Notes in Mathematics, Nonlinear Analysis and Mechanics. Heriot-Symposium 4 (1979) 136-212.

[29] J.D. Towers, Convergence of a finite difference scheme for conservation laws with a discontinous flux. SIAM. J. Numer. Anal. 38 (2000) 681-698.

[30] C.J. van Duijn, L.A. Peletier and I.S. Pop, A new class of entropy solutions of the Buckley-Leverett equation. SIAM J. Math. Anal. 39 (2007) 507-536.

[31] A. Vasseur, Strong traces for solutions of multidimensional scalar conservation laws. Arch. Rational Mech. Anal. 160 (2001) $181-193$.

[32] A.I. Vol'pert, Generalized solutions of degenerate second-order quasilinear parabolic and elliptic equations. Adv. Differ. Equ. 5 (2000) 1493-1518.

[33] C.C. Wu, New theory of MHD shock waves, in Viscous Profiles and Numerical Methods for Shock Waves, edited by M. Shearer. SIAM, Philadelphia, PA (1991) 209-236. 